\title{
The Synthesis of Naphthalenyl Triflates via the Cationic Annulation of Benzodiynes with Triflic Acid
}

\author{
Chenxin Ge, ${ }^{\mathrm{a}}$ Guohua Wang, ${ }^{\mathrm{a}}$ Panpan $\mathrm{Wu},{ }^{\mathrm{b}}$ Chao Chen * a, b \\ a C. X. Ge, G. H. Wang, Prof. Dr. C. Chen \\ Key Laboratory of Bioorganic Phosphorus Chemistry \& Chemical Biology (Ministry of Education), Department of \\ Chemistry, Tsinghua University, Beijing 100084, China; E-mail: chenchao01@mails.tsinghua.edu.cn \\ b Prof. Dr. P. Wu, Prof. Dr. C. Chen \\ School of Chemical \& Environmental Engineering, Wuyi University, Jiangmen, 529000, China; International \\ Healthcare Innovation Institute (Jiangmen), Jiangmen, 529000, China
}

\section{List of the contents:}

General information................................................. 2

General procedure for the preparation of starting materials benzodiynes 1..........S3 - S11

General procedure for the preparation of desired product $2 \ldots \ldots \ldots \ldots \ldots \ldots . \mathrm{S} 12-\mathrm{S} 19$

To test other benzodiyne-type analogues under standard conditions............S20

Scale synthesis of naphthalenyl triflate 2a from $10 \mathrm{mmol}$ of substrate $\mathbf{1 a} .$. S21

Mechanistic studies $. \mathrm{S} 22-\mathrm{S} 23$

Derivatization of naphthalenyl triflates. $\mathrm{S} 24-\mathrm{S} 26$

References. S26

NMR spectra. S27 - S59 


\section{General information}

All the reactions were carried out in oven-dried screw-capped tube with a Teflon-lined septum under $\mathrm{N}_{2}$ atmosphere. Benzodiyne reagents were prepared according to the literatures. ${ }^{1}$ Trifluoromethanesulfonic acid (TfOH) was purchased from Acros $(99 \%)$ or Adamas $(99 \%+)$ and used as supplied. All of the solvents were fresh distilled according to standard method before use. Column chromatography was performed on silica gel (particle size 10-40 $\mu \mathrm{m}$, Ocean Chemical Factory of Qingdao, China). ${ }^{1} \mathrm{H}$ NMR ${ }^{13} \mathrm{C}$ NMR and ${ }^{19} \mathrm{~F}$ NMR spectra were recorded on a AL-400MHz AL-600MHz spectrometer at ambient temperature with $\mathrm{CDCl}_{3}$ as the solvent. ${ }^{1} \mathrm{HNMR}$ spectra are reported as follows: chemical shift in $\mathrm{ppm}(\delta)$ relative to the chemical shift of $\mathrm{CDCl}_{3}$ at $7.26 \mathrm{ppm}$, integration, multiplicities ( $\mathrm{s}=$ singlet, $\mathrm{d}=$ doublet, $\mathrm{t}=$ triplet, $\mathrm{q}$ $=$ quartet, $\mathrm{m}=$ multiplet) and coupling constants $(\mathrm{Hz}) \cdot{ }^{13} \mathrm{C}$ NMR spectra are reported in ppm $(\delta)$ relative to the central line of triplet for $\mathrm{CDCl}_{3}$ at $77.16 \mathrm{ppm}$. HRMS spectra were obtained using Bruker Esquire ion trap mass spectrometer in negative mode. The reaction progress was monitored by GC using n-dodecane as internal standard. 


\section{Experimental section}

\subsection{General procedure for the preparation of starting materials benzodiynes}

General Procedure for Sonogashira cross coupling of aryl iodides with different substituted acetylenes (8):<smiles>[R]c1cc(Br)c(I)cc1[R]</smiles>

3

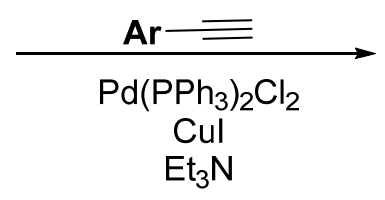

$\mathrm{Et}_{3} \mathrm{~N}$<smiles>[R]c1cc(Br)c(C#CBr)cc1[R]</smiles>

8

A suspension of 2-bromoiodobenzene 3 (10 mmol), $\mathrm{PdCl}_{2}\left(\mathrm{PPh}_{3}\right)_{2}(0.5 \mathrm{mmol}), \mathrm{Cu}(\mathrm{I})$ iodide $(0.5 \mathrm{mmol})$ in $50 \mathrm{~mL}$ of triethylamine was degassed three times with freeze/pump/thaw technique in a flame-dried round bottom flask. 1.1 Equiv. of alkyne (11 mmol) was added using a syringe to the thawed solution under nitrogen. The reaction was allowed to react for 8 hours and monitored by TLC. After total consumption of the aryl iodide, the reaction mixture was filtered through celite and extracted with EtOAc $(3 \times 30 \mathrm{~mL})$. The organic layer was washed with a saturated solution of ammonium chloride $(2 \times 30 \mathrm{~mL})$, brine $(2 \times 30 \mathrm{~mL})$ and dried over anhydrous $\mathrm{Na}_{2} \mathrm{SO}_{4}$. Solvent was removed in vacuo. The reaction mixture was purified by flash chromatography on silica gel (eluent: PE/EtOAc) to afford compound 8.

General Procedure for Sonogashira cross coupling of aryl bromides with ethynyltrimethylsilane (9):

A suspension of aryl bromide $(5 \mathrm{mmol}), \mathrm{PdCl}_{2}(\mathrm{PhCN})_{2}(0.25 \mathrm{mmol}), \mathrm{Cu}(\mathrm{I})$ iodide $(0.25 \mathrm{mmol})$ in $20 \mathrm{~mL}$ of triethylamine was degassed three times with freeze/pump/thaw technique in a flame dried round bottom flask. Once reaction mixture was completely thawed and the atmosphere replaced with nitrogen, tritert-butylphosphine $(0.5 \mathrm{mmol})$ in a $10 \%$ solution of toluene was added, immediately followed by 1.2 equiv. of ethynyltrimethylsilane $(6 \mathrm{mmol})$ using a syringe. The reaction was allowed to react for 8 hours and monitored by TLC. After total consumption of the aryl bromide, the reaction mixture was filtered through celite and extracted with EtOAc $(3 \times 30 \mathrm{~mL})$. The organic layer was washed with a saturated solution of ammonium chloride $(2 \times 30 \mathrm{~mL})$, brine $(2 \times 30 \mathrm{~mL})$ and dried over anhydrous $\mathrm{Na}_{2} \mathrm{SO}_{4}$. Solvent was removed in vacuo. The reaction mixture was purified by flash chromatography (eluent: PE/EA) on silica gel to afford compound $\mathbf{9}$.<smiles>[R]c1cc(Br)c(C#CBr)cc1[R]</smiles>

8

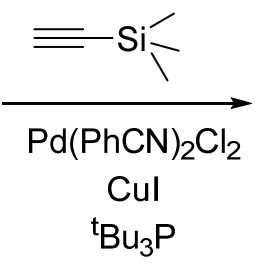

$\mathrm{Et}_{3} \mathrm{~N}$<smiles>[R]c1cc(C#CBr)c(C#C[Si](C)(C)C)cc1[R]</smiles>

9 


\section{General Procedure for deprotection of TMS protecting groups (1):}<smiles>[R]c1cc(C#C[Bi])c(C#C[Si](C)(C)C)cc1[R]</smiles>

9

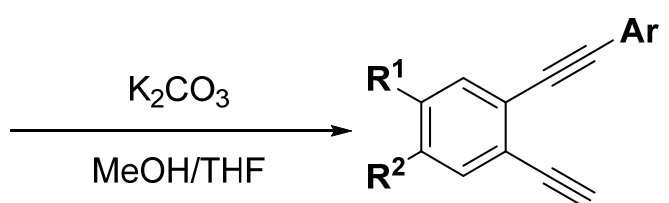

To a solution of benzodiyne 9 ( $2 \mathrm{mmol})$, in 1:1 mixture of MeOH/THF (30 mL) was added $\mathrm{K}_{2} \mathrm{CO}_{3}(2 \mathrm{mmol})$. The solution was stirred at room temperature for $8 \mathrm{~h}$ under nitrogen. Water was added to quench the reaction and an aqueous work up was performed. The reaction mixture was purified by flash chromatography (eluent: $\mathrm{PE} / \mathrm{EA})$ on silica gel to afford compound $\mathbf{1}$.

\section{1-ethynyl-2-(phenylethynyl)benzene (1a)}<smiles>C#Cc1ccccc1C#Cc1ccccc1</smiles>

Chromatographic purification (petroleum ether) afforded compound 1a (98\% yield) as a light yellow oil. $\mathrm{R}_{f}=0.3$ (petroleum ether); ${ }^{1} \mathrm{H}$ NMR (400 MHz, Chloroform-d) $\delta$ $7.68-7.52(\mathrm{~m}, 4 \mathrm{H}), 7.44-7.27(\mathrm{~m}, 5 \mathrm{H}), 3.43(\mathrm{~s}, 1 \mathrm{H}) .{ }^{13} \mathrm{C}$ NMR $(101 \mathrm{MHz}$, Chloroform-d) $\delta 132.7,131.9,128.6,128.4,128.0,126.4,124.7,123.2,93.7,88.0$, 82.3, 81.3.

\section{1-ethynyl-2-(p-tolylethynyl)benzene (1b)}<smiles>C#Cc1ccccc1C#Cc1ccc(C)cc1</smiles>

Chromatographic purification (petroleum ether) afforded compound $1 \mathrm{~b}$ (93\% yield) as a light yellow oil. $\mathrm{R}_{f}=0.3$ (petroleum ether); ${ }^{1} \mathrm{H}$ NMR (400 MHz, Chloroform-d) $\delta$ $7.53(\mathrm{dd}, \mathrm{J}=7.4,1.7 \mathrm{~Hz}, 2 \mathrm{H}), 7.47(\mathrm{~d}, \mathrm{~J}=8.0 \mathrm{~Hz}, 2 \mathrm{H}), 7.33(\mathrm{td}, \mathrm{J}=7.6,1.8 \mathrm{~Hz}, 1 \mathrm{H})$, $7.30-7.25(\mathrm{~m}, 1 \mathrm{H}), 7.17(\mathrm{~d}, \mathrm{~J}=8.0 \mathrm{~Hz}, 2 \mathrm{H}), 3.36(\mathrm{~s}, 1 \mathrm{H}), 2.38(\mathrm{~s}, 3 \mathrm{H}) .{ }^{13} \mathrm{C} \mathrm{NMR}$ (101 MHz, Chloroform-d) $\delta$ 138.8, 132.7, 131.8, 131.8, 129.2, 128.7, 127.9, 126.7, 124.6, 120.2, 94.0, 87.4, 82.4, 81.2, 21.7. 
<smiles>C#Cc1ccccc1C#Cc1ccc(CC)cc1</smiles>

Chromatographic purification (petroleum ether) afforded compound 1c (84\% yield) as a light yellow oil. $\mathrm{R}_{f}=0.3$ (petroleum ether); ${ }^{1} \mathrm{H}$ NMR $(400 \mathrm{MHz}$, Chloroform-d) $\delta$ $7.53(\mathrm{dd}, \mathrm{J}=7.4,1.6 \mathrm{~Hz}, 2 \mathrm{H}), 7.50(\mathrm{~d}, \mathrm{~J}=8.0 \mathrm{~Hz}, 2 \mathrm{H}), 7.33(\mathrm{td}, \mathrm{J}=7.5,1.6 \mathrm{~Hz}, 1 \mathrm{H})$, $7.30-7.25(\mathrm{~m}, 1 \mathrm{H}), 7.19(\mathrm{~d}, \mathrm{~J}=8.0 \mathrm{~Hz}, 2 \mathrm{H}), 3.36(\mathrm{~s}, 1 \mathrm{H}), 2.67$ (q, J = 7.5 Hz, 2H), $1.25(\mathrm{t}, \mathrm{J}=7.6 \mathrm{~Hz}, 3 \mathrm{H}) .{ }^{13} \mathrm{C}$ NMR (101 MHz, Chloroform-d) $\delta 145.1,132.7,131.9$, $131.8,128.7,128.1,127.9,126.7,124.7,120.5,94.0,87.4,82.4,81.2,29.0,15.5$.

\section{1-((4-(tert-butyl)phenyl)ethynyl)-2-ethynylbenzene (1d)}<smiles>C#Cc1ccccc1C#Cc1ccc(C(C)(C)C)cc1</smiles>

Chromatographic purification (petroleum ether) afforded compound 1d (99\% yield) as a light yellow solid. $\mathrm{R}_{f}=0.3$ (petroleum ether); ${ }^{1} \mathrm{H}$ NMR $(400 \mathrm{MHz}$, Chloroform-d) $\delta 7.52(\mathrm{dd}, \mathrm{J}=9.6,7.8 \mathrm{~Hz}, 4 \mathrm{H}), 7.41-7.36(\mathrm{~m}, 2 \mathrm{H}), 7.32(\mathrm{td}, \mathrm{J}=7.5,1.6 \mathrm{~Hz}, 1 \mathrm{H})$, $7.27(\mathrm{td}, \mathrm{J}=7.5,1.5 \mathrm{~Hz}, 1 \mathrm{H}), 3.35(\mathrm{~s}, 1 \mathrm{H}), 1.33(\mathrm{~s}, 9 \mathrm{H}) .{ }^{13} \mathrm{C}$ NMR $(101 \mathrm{MHz}$, Chloroform-d) $\delta 152.0,132.7,131.9,131.7,128.6,127.9,126.7,125.5,124.7,120.3$, 93.9, 87.4, 82.4, 81.2, 35.0, 31.3.

\section{1-(cyclopropylethynyl)-2-ethynylbenzene (1e)}<smiles>C#Cc1ccccc1C#CC1CC1</smiles>

Chromatographic purification (petroleum ether) afforded compound $1 \mathrm{e}$ (78\% yield) as a light yellow oil. $\mathrm{R}_{f}=0.3$ (petroleum ether); ${ }^{1} \mathrm{H}$ NMR (400 MHz, Chloroform-d) $\delta$ 7.46 (dd, J = 7.4, 1.6 Hz, 1H), 7.38 (dd, J = 7.5, 1.6 Hz, 1H), 7.25 (td, J = 7.6, 1.7 Hz, 1H), $7.20(\mathrm{td}, \mathrm{J}=7.6,1.7 \mathrm{~Hz}, 1 \mathrm{H}), 3.28(\mathrm{~s}, 1 \mathrm{H}), 1.55-1.46(\mathrm{~m}, 1 \mathrm{H}), 0.94-0.81(\mathrm{~m}$, 4H). ${ }^{13} \mathrm{C}$ NMR (101 MHz, Chloroform-d) $\delta$ 132.6, 131.9, 128.5, 127.2, 127.1, 124.5, 98.4, 82.5, 80.6, 74.3, 9.1, 0.6. 
<smiles>C#Cc1ccccc1C#Cc1ccc(F)cc1</smiles>

Chromatographic purification (petroleum ether) afforded compound $1 \mathrm{f}$ (96\% yield) as a light yellow oil. $\mathrm{R}_{f}=0.3$ (petroleum ether); ${ }^{1} \mathrm{H}$ NMR (400 MHz, Chloroform-d) $\delta$ $7.59-7.55(\mathrm{~m}, 2 \mathrm{H}), 7.55-7.52(\mathrm{~m}, 2 \mathrm{H}), 7.31(\mathrm{dtd}, \mathrm{J}=17.0,7.6,1.7 \mathrm{~Hz}, 2 \mathrm{H}), 7.09-$ $7.03(\mathrm{~m}, 2 \mathrm{H}), 3.38(\mathrm{~s}, 1 \mathrm{H}) .{ }^{13} \mathrm{C}$ NMR $(101 \mathrm{MHz}$, Chloroform-d) $\delta 162.8(\mathrm{~d}, \mathrm{~J}=249.6$ $\mathrm{Hz}), 133.8(\mathrm{~d}, \mathrm{~J}=8.5 \mathrm{~Hz}), 132.7,131.8,128.7,128.1,126.2,124.7,119.4$ (d, J = 3.7 $\mathrm{Hz}), 115.8(\mathrm{~d}, \mathrm{~J}=22.1 \mathrm{~Hz}), 92.6,87.7,82.3,81.3$.

\section{1-((4-bromophenyl)ethynyl)-2-ethynylbenzene (1g)}<smiles>C#Cc1ccccc1C#Cc1ccc(Br)cc1</smiles>

Chromatographic purification (petroleum ether) afforded compound $1 \mathrm{~g}$ (79 \% yield) as a colorless oil. $\mathrm{R}_{f}=0.3$ (petroleum ether); ${ }^{1} \mathrm{H}$ NMR (400 MHz, Chloroform-d) $\delta$ 7.54 (ddd, J = 6.9, 4.6, $1.8 \mathrm{~Hz}, 2 \mathrm{H}), 7.51-7.46(\mathrm{~m}, 2 \mathrm{H}), 7.45-7.38(\mathrm{~m}, 2 \mathrm{H}), 7.32$ (m, 2H), 3.37 (s, 1H). ${ }^{13} \mathrm{C}$ NMR (101 MHz, Chloroform-d) $\delta 133.3,132.8,131.9,131.8$, $128.7,128.3,126.1,124.8,122.9,122.2,92.5,89.1,82.2,81.4$.

\section{1-((4-chlorophenyl)ethynyl)-2-ethynylbenzene (1h)}<smiles>C#Cc1ccccc1C#Cc1ccc(Cl)cc1</smiles>

Chromatographic purification (petroleum ether) afforded compound $1 \mathrm{~h}$ (98\% yield) as a light yellow oil. $\mathrm{R}_{f}=0.3$ (petroleum ether); ${ }^{1} \mathrm{H}$ NMR (400 MHz, Chloroform-d) $\delta$ $7.52(\mathrm{~m}, 4 \mathrm{H}), 7.37-7.27(\mathrm{~m}, 4 \mathrm{H}), 3.36(\mathrm{~s}, 1 \mathrm{H}) .{ }^{13} \mathrm{C}$ NMR (101 MHz, Chloroform-d) $\delta 134.7,133.1,132.8,131.9,128.8,128.7,128.3,126.1,124.8,121.8,92.5,88.9$, 82.3, 81.4. 
<smiles>C#Cc1ccccc1C#Cc1ccccc1Cl</smiles>

Chromatographic purification (petroleum ether) afforded compound $1 \mathrm{i}$ (87\% yield) as a light yellow solid. $\mathrm{R}_{f}=0.3$ (petroleum ether); ${ }^{1} \mathrm{H}$ NMR (400 MHz, Chloroform-d) $\delta$ 7.57 (td, J = 6.3, 5.9, 2.1 Hz, 2H), $7.52(\mathrm{dd}, \mathrm{J}=7.3,1.8 \mathrm{~Hz}, 1 \mathrm{H}), 7.40$ (dd, J = 7.0, 2.4 $\mathrm{Hz}, 1 \mathrm{H}), 7.34-7.24$ (m, 2H), $7.25-7.17$ (m, 2H). 3.37 (s, 1H). ${ }^{13} \mathrm{C}$ NMR (101 MHz, Chloroform-d) $\delta 136.0133 .7,132.7,132.2,129.6,129.4,128.6,128.4,126.5,126.1$, $124.7,123.2,92.9,90.2,82.2,81.6$.

1-((3-chlorophenyl)ethynyl)-2-ethynylbenzene (1j)<smiles>C#Cc1ccccc1C#Cc1cccc(Cl)c1</smiles>

Chromatographic purification (petroleum ether) afforded compound $1 \mathrm{j}$ (99\% yield) as a light yellow oil. $\mathrm{R}_{f}=0.3$ (petroleum ether); ${ }^{1} \mathrm{H}$ NMR (400 MHz, Chloroform-d) $\delta$ $7.63-7.49(\mathrm{~m}, 3 \mathrm{H}), 7.46(\mathrm{dd}, \mathrm{J}=7.5,1.7 \mathrm{~Hz}, 1 \mathrm{H}), 7.32(\mathrm{~m}, 4 \mathrm{H}), 3.40(\mathrm{~s}, 1 \mathrm{H}) .{ }^{13} \mathrm{C}$ NMR (101 MHz, Chloroform-d) $\delta$ 134.3, 132.7, 132.0, 131.6, 130.0, 129.7, 128.8, $128.7,128.4,125.8,124.9,124.9,92.1,89.1,82.1,81.5$.

\section{1-ethynyl-2-((4-(trifluoromethyl)phenyl)ethynyl)benzene (1k)}<smiles>C#Cc1ccccc1C#Cc1ccc(C(F)(F)F)cc1</smiles>

Chromatographic purification (petroleum ether) afforded compound $1 \mathrm{k}$ (98\% yield) as a light yellow solid. $\mathrm{R}_{f}=0.3$ (petroleum ether); ${ }^{1} \mathrm{H}$ NMR $(400 \mathrm{MHz}$, Chloroform-d) $\delta 7.67(\mathrm{~d}, \mathrm{~J}=8.3 \mathrm{~Hz}, 2 \mathrm{H}), 7.61(\mathrm{~d}, \mathrm{~J}=8.2 \mathrm{~Hz}, 2 \mathrm{H}), 7.59-7.53(\mathrm{~m}, 2 \mathrm{H}), 7.34(\mathrm{tt}, \mathrm{J}=$ 7.4, $5.7 \mathrm{~Hz}, 2 \mathrm{H}), 3.40$ (s, 1H). ${ }^{13} \mathrm{C}$ NMR (101 MHz, Chloroform-d) $\delta$ 132.8, 132.1, 132.0, 130.2 (q, J = 32.7 Hz).128.8, 128.6, 127.1, 125.7, 125.4 (q, J = 3.7 Hz), 125.0, 124.1 (q, J = 272.2 Hz), 92.1, 90.3, 82.1, 81.6. 


\section{methyl 4-((2-ethynylphenyl)ethynyl)benzoate (1I)}<smiles>C#Cc1ccccc1C#Cc1ccc(C(C)=O)cc1</smiles>

Chromatographic purification (petroleum ether : ethyl acetate $=20: 1$ ) afforded compound 11 (98\% yield) as a yellow solid. $\mathrm{R}_{f}=0.3$ (petroleum ether : ethyl acetate = $20: 1) ;{ }^{1} \mathrm{H}$ NMR (400 MHz, Chloroform-d) $\delta 8.02(\mathrm{~d}, \mathrm{~J}=8.2 \mathrm{~Hz}, 2 \mathrm{H}), 7.61(\mathrm{~d}, \mathrm{~J}=$ $8.2 \mathrm{~Hz}, 2 \mathrm{H}), 7.56-7.48$ (m, 2H), 7.32 (qt, J = 7.3, 3.7 Hz, 2H), 3.92 (s, 3H), 3.40 (s, $1 \mathrm{H}) .{ }^{13} \mathrm{C}$ NMR (101 MHz, Chloroform-d) $\delta 166.6,132.8,132.0,131.7,129.8,129.6$, $128.7,128.5,127.9,125.8,124.9,92.7,90.8,82.1,81.6,52.3$.

\section{4-((2-ethynylphenyl)ethynyl)-1,1'-biphenyl (1m)}<smiles>C#Cc1ccccc1C#Cc1ccc(-c2ccccc2)cc1</smiles>

Chromatographic purification (petroleum ether) afforded compound $1 \mathrm{~m}$ (96\% yield) as a yellow solid. $\mathrm{R}_{f}=0.3$ (petroleum ether); ${ }^{1} \mathrm{H}$ NMR (400 MHz, Chloroform-d) $\delta$ $7.67-7.53(\mathrm{~m}, 8 \mathrm{H}), 7.49-7.42(\mathrm{~m}, 2 \mathrm{H}), 7.40-7.27(\mathrm{~m}, 3 \mathrm{H}), 3.39(\mathrm{~s}, 1 \mathrm{H}) .{ }^{13} \mathrm{C} \mathrm{NMR}$ (101 MHz, Chloroform-d) $\delta 141.4,140.5,132.8,132.3,131.9,129.0,128.7,128.1$, $127.8,127.2,126.5,124.8,122.2,93.6,88.7,82.4,81.3$.

\section{3-((2-ethynylphenyl)ethynyl)thiophene (1n)}<smiles>C#Cc1ccccc1C#Cc1ccsc1</smiles>

Chromatographic purification (petroleum ether) afforded compound $1 \mathrm{n}$ (60\% yield) as a light yellow oil. $\mathrm{R}_{f}=0.2$ (petroleum ether); ${ }^{1} \mathrm{H}$ NMR (400 MHz, Chloroform-d) $\delta$ $7.55(\mathrm{~d}, \mathrm{~J}=3.1 \mathrm{~Hz}, 1 \mathrm{H}), 7.52(\mathrm{dt}, \mathrm{J}=7.0,2.3 \mathrm{~Hz}, 2 \mathrm{H}), 7.35-7.24(\mathrm{~m}, 3 \mathrm{H}), 7.22$ (d, J $=4.9 \mathrm{~Hz}, 1 \mathrm{H}), 3.34(\mathrm{~s}, 1 \mathrm{H}) .{ }^{13} \mathrm{C}$ NMR $(101 \mathrm{MHz}$, Chloroform-d) $\delta 132.7,131.9,130.1$, 129.2, 128.7, 128.0, 126.4, 125.5, 124.6, 122.3, 88.8, 87.5, 82.3, 81.2. 
<smiles>C#Cc1ccc(F)cc1C#Cc1ccccc1</smiles>

Chromatographic purification (petroleum ether) afforded compound 10 (97\% yield) as a light yellow oil. $\mathrm{R}_{f}=0.3$ (petroleum ether); ${ }^{1} \mathrm{H}$ NMR (400 MHz, Chloroform-d) $\delta$ $7.66-7.53(\mathrm{~m}, 2 \mathrm{H}), 7.49(\mathrm{dd}, \mathrm{J}=8.6,5.6 \mathrm{~Hz}, 1 \mathrm{H}), 7.35(\mathrm{q}, \mathrm{J}=3.1 \mathrm{~Hz}, 3 \mathrm{H}), 7.22(\mathrm{dd}$, $\mathrm{J}=9.2,2.8 \mathrm{~Hz}, 1 \mathrm{H}), 6.98(\mathrm{td}, \mathrm{J}=8.3,2.6 \mathrm{~Hz}, 1 \mathrm{H}), 3.33(\mathrm{~s}, 1 \mathrm{H}) .{ }^{13} \mathrm{C} \mathrm{NMR}(101 \mathrm{MHz}$, Chloroform-d) $\delta 162.2(\mathrm{~d}, \mathrm{~J}=250.6 \mathrm{~Hz}), 134.5(\mathrm{~d}, \mathrm{~J}=8.8 \mathrm{~Hz}), 132.0,129.0,128.5$, 128.4, 122.8, $121.0(\mathrm{~d}, \mathrm{~J}=3.7 \mathrm{~Hz}), 118.7(\mathrm{~d}, \mathrm{~J}=23.3 \mathrm{~Hz}), 115.8(\mathrm{~d}, \mathrm{~J}=22.3 \mathrm{~Hz}), 94.7$, $86.9,81.4,81.0$.

\section{2-ethynyl-4-fluoro-1-(phenylethynyl)benzene (1p)}<smiles>C#Cc1cc(F)ccc1C#Cc1ccccc1</smiles>

Chromatographic purification (petroleum ether) afforded compound $1 \mathrm{p}$ (80\% yield) as a brownish oil. $\mathrm{R}_{f}=0.3$ (petroleum ether); ${ }^{1} \mathrm{H}$ NMR (400 MHz, Chloroform-d) $\delta$ $7.59-7.47$ (m, 3H), 7.34 (q, J = 3.1, 2.5 Hz, 3H), $7.22(\mathrm{dd}, \mathrm{J}=9.0,2.8 \mathrm{~Hz}, 1 \mathrm{H}), 7.03$ $(\mathrm{td}, \mathrm{J}=8.3,2.6 \mathrm{~Hz}, 1 \mathrm{H}), 3.40(\mathrm{~s}, 1 \mathrm{H}) .{ }^{13} \mathrm{C}$ NMR $(101 \mathrm{MHz}$, Chloroform-d) $\delta 161.7$ (d, $\mathrm{J}=250.0 \mathrm{~Hz}), 133.7(\mathrm{~d}, \mathrm{~J}=8.6 \mathrm{~Hz}), 131.8,128.7,128.5,126.6(\mathrm{~d}, \mathrm{~J}=10.2 \mathrm{~Hz}), 123.1$, $122.8(\mathrm{~d}, \mathrm{~J}=3.6 \mathrm{~Hz}), 119.6(\mathrm{~d}, \mathrm{~J}=23.8 \mathrm{~Hz}), 116.5(\mathrm{~d}, \mathrm{~J}=22.0 \mathrm{~Hz}), 93.3,87.0,82.4$, 81.2 .

\section{4-chloro-2-ethynyl-1-(phenylethynyl)benzene (1q)}<smiles>C#Cc1cc(Cl)ccc1C#Cc1ccccc1</smiles>

Chromatographic purification (petroleum ether) afforded compound 1q (93\% yield) as a light yellow oil. $\mathrm{R}_{f}=0.3$ (petroleum ether); ${ }^{1} \mathrm{H}$ NMR $(400 \mathrm{MHz}$, Chloroform-d) $\delta$ $7.56(\mathrm{tt}, \mathrm{J}=4.9,2.4 \mathrm{~Hz}, 2 \mathrm{H}), 7.53-7.50(\mathrm{~m}, 1 \mathrm{H}), 7.46(\mathrm{dd}, \mathrm{J}=8.4,1.8 \mathrm{~Hz}, 1 \mathrm{H}), 7.40$ $-7.33(\mathrm{~m}, 3 \mathrm{H}), 7.31(\mathrm{dt}, \mathrm{J}=8.4,2.1 \mathrm{~Hz}, 1 \mathrm{H}), 3.41(\mathrm{~s}, 1 \mathrm{H}) .{ }^{13} \mathrm{C}$ NMR $(101 \mathrm{MHz}$, Chloroform-d) $\delta 133.9,132.9,132.5,131.9,129.1,128.9,128.5,126.2,125.0,123.0$, 94.6, 87.0, 82.5, 81.1. 
<smiles>C#Cc1cc(C)ccc1C#Cc1ccccc1</smiles>

Chromatographic purification (petroleum ether) afforded compound $1 \mathrm{r}$ (87\% yield) as a light yellow oil. $\mathrm{R}_{f}=0.3$ (petroleum ether); ${ }^{1} \mathrm{H}$ NMR (400 MHz, Chloroform-d) $\delta$ $7.58-7.53(\mathrm{~m}, 2 \mathrm{H}), 7.43(\mathrm{~d}, \mathrm{~J}=8.0 \mathrm{~Hz}, 1 \mathrm{H}), 7.38-7.31(\mathrm{~m}, 4 \mathrm{H}), 7.14$ (dd, J = 8.0, $1.8 \mathrm{~Hz}, 1 \mathrm{H}), 3.33(\mathrm{~s}, 1 \mathrm{H}), 2.35(\mathrm{~s}, 3 \mathrm{H}) .{ }^{13} \mathrm{C}$ NMR (101 MHz, Chloroform-d) $\delta 138.3$, 133.3, 131.9, 131.8, 129.7, 128.4, 124.6, 123.5, 93.0, 88.1, 82.5, 80.8, 21.4.

\section{2-ethynyl-4-methoxy-1-(phenylethynyl)benzene (1s)}<smiles>C#Cc1cc(OC)ccc1C#Cc1ccccc1</smiles>

Chromatographic purification (petroleum ether) afforded compound $1 \mathrm{~s}$ (79\% yield) as a light brownish solid. $\mathrm{R}_{f}=0.1$ (petroleum ether); ${ }^{1} \mathrm{H}$ NMR (400 MHz, Chloroform-d) $\delta 7.55$ (dd, J = 7.5, 2.2 Hz, 2H), 7.46 (d, J = 8.7 Hz, 1H), 7.34 (dq, J = 8.5, 2.8, 2.3 Hz, 3H), $7.05(\mathrm{~d}, \mathrm{~J}=2.7 \mathrm{~Hz}, 1 \mathrm{H}), 6.89(\mathrm{dd}, \mathrm{J}=8.7,2.7 \mathrm{~Hz}, 1 \mathrm{H}), 3.83$ (s, $3 \mathrm{H}), 3.36(\mathrm{~s}, 1 \mathrm{H}) .{ }^{13} \mathrm{C}$ NMR (101 MHz, Chloroform-d) $\delta 159.2,133.3,131.7,128.4$, $128.3,126.0,123.6,118.9,117.4,115.7,92.2,87.9,82.3,81.1,55.6$.

\section{1-ethynyl-2-((4-methoxyphenyl)ethynyl)benzene (1t)}<smiles>C#Cc1ccccc1C#Cc1ccc(OC)cc1</smiles>

Chromatographic purification (petroleum ether) afforded compound $1 \mathrm{t}$ (91\% yield) as a yellow solid. $\mathrm{R}_{f}=0.1$ (petroleum ether); ${ }^{1} \mathrm{H}$ NMR (400 MHz, Chloroform-d) $\delta 7.56$ -7.47 (m, 4H), 7.30 (t, J = 7.6 Hz, 1H), 7.24 (t, J = 7.6 Hz, 1H), 6.87 (dd, J = 9.0, 2.4 $\mathrm{Hz}, 2 \mathrm{H}), 3.79(\mathrm{~s}, 3 \mathrm{H}), 3.38(\mathrm{~s}, 1 \mathrm{H}) .{ }^{13} \mathrm{C}$ NMR (101 MHz, Chloroform-d) $\delta 159.9$, 133.3, 132.6, 131.6, 128.6, 127.6, 126.7, 124.4, 115.3, 114.1, 93.8, 86.7, 82.4, 81.1, 55.3 . 


\section{1,2-bis(phenylethynyl)benzene (1u)}<smiles>C(#Cc1ccccc1C#Cc1ccccc1)c1ccccc1</smiles>

Chromatographic purification (petroleum ether) afforded compound $1 \mathrm{u}$ (95\% yield) as a yellow solid. $\mathrm{R}_{f}=0.3$ (petroleum ether); ${ }^{1} \mathrm{H}$ NMR $(400 \mathrm{MHz}$, Chloroform-d) $\delta$ $7.57(\mathrm{dt}, \mathrm{J}=5.4,3.0 \mathrm{~Hz}, 6 \mathrm{H}), 7.39-7.28(\mathrm{~m}, 8 \mathrm{H}) .{ }^{13} \mathrm{C}$ NMR $(101 \mathrm{MHz}$, Chloroform-d) $\delta 131.9,131.8,128.6,128.5,128.2,126.0,123.5,93.7,88.5$.

\section{1,2-di(hex-1-yn-1-yl)benzene (lv)<smiles>CCCCC#Cc1ccccc1C#CCCCC</smiles>

Chromatographic purification (petroleum ether) afforded compound $1 \mathrm{v}$ (93\% yield) as a light yellow oil. $\mathrm{R}_{f}=0.3$ (petroleum ether); ${ }^{1} \mathrm{H}$ NMR (400 MHz, Chloroform-d) $\delta$ $7.41-7.34(\mathrm{~m}, 2 \mathrm{H}), 7.21-7.13(\mathrm{~m}, 2 \mathrm{H}), 2.48(\mathrm{t}, \mathrm{J}=6.9 \mathrm{~Hz}, 4 \mathrm{H}), 1.67-1.59(\mathrm{~m}, 4 \mathrm{H})$, $1.58-1.49(\mathrm{~m}, 4 \mathrm{H}), 0.97(\mathrm{t}, \mathrm{J}=7.2 \mathrm{~Hz}, 6 \mathrm{H}) .{ }^{13} \mathrm{C}$ NMR (101 MHz, Chloroform-d) $\delta$ $131.9,127.2,126.5,94.1,79.7,31.0,22.1,19.4,13.8$.

\section{1-ethynyl-2-(hex-1-yn-1-yl)benzene (1w)}<smiles>C#Cc1ccccc1C#CCCCC</smiles>

Chromatographic purification (petroleum ether) afforded compound $1 \mathrm{w}$ ( $85 \%$ yield) as a light yellow oil. $\mathrm{R}_{f}=0.3$ (petroleum ether); ${ }^{1} \mathrm{H}$ NMR $(400 \mathrm{MHz}$, Chloroform-d) $\delta$ $7.47(\mathrm{dd}, \mathrm{J}=7.7,1.6 \mathrm{~Hz}, 1 \mathrm{H}), 7.40(\mathrm{dd}, \mathrm{J}=7.5,1.5 \mathrm{~Hz}, 1 \mathrm{H}), 7.26(\mathrm{td}, \mathrm{J}=7.6,1.6 \mathrm{~Hz}$, 1H), $7.21(\mathrm{td}, \mathrm{J}=7.6,1.6 \mathrm{~Hz}, 1 \mathrm{H}), 3.27(\mathrm{~s}, 1 \mathrm{H}), 2.48(\mathrm{t}, \mathrm{J}=6.9 \mathrm{~Hz}, 2 \mathrm{H}), 1.68-1.58$ $(\mathrm{m}, 2 \mathrm{H}), 1.57-1.49(\mathrm{~m}, 2 \mathrm{H}), 0.95(\mathrm{t}, \mathrm{J}=7.2 \mathrm{~Hz}, 3 \mathrm{H}) .{ }^{13} \mathrm{C}$ NMR (101 MHz, Chloroform-d) $\delta 132.6,132.0,128.6,127.3,124.5,95.2,82.6,80.5,79.2,30.9,22.1$, $19.4,13.8$. 


\subsection{General procedure for the preparation of desired product}

To a solution of the appropriate benzodiyne $1(0.2 \mathrm{mmol})$ in freshly distilled $\mathrm{CHCl}_{3}$ $(10 \mathrm{~mL})$ under $\mathrm{N}_{2}$ atmosphere was added trifluoromethanesulfonic acid (18 $\mu \mathrm{L}, 0.2$ $\mathrm{mmol})$ at room temperature. The resulting dark brown reaction mixture was then stirred at $50{ }^{\circ} \mathrm{C}$ until complete consumption of starting material was observed by TLC (For convenience, about $12 \mathrm{~h}$ ). On completion the reaction was quenched with water, extracted with EA. The organic layer was dried over $\mathrm{Na}_{2} \mathrm{SO}_{4}$, filtered, and concentrated. The crude residue was finally purified by column chromatography over silica gel using petroleum ether/ethyl acetate as eluent to yield the desired title compound 2.<smiles>[R]c1ccc(C#Cc2cc([R])c([R])cc2C#C)cc1</smiles>

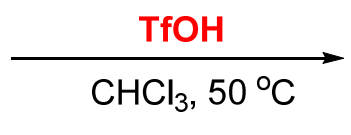

1<smiles>[R]c1ccc(-c2cc([OH+])c3cc([R4])c([R])cc3c2)cc1</smiles>

\section{3-phenylnaphthalen-1-yl trifluoromethanesulfonate (2a):}<smiles>CCCc1cc(-c2ccccc2)cc2ccccc12</smiles>

Purification by flash chromatography on silica gel (petroleum ether) provided $2 \mathrm{a}$ as a light yellow oil; $\mathrm{R}_{f}=0.2$ (petroleum ether); $56 \mathrm{mg}$, isolated yield: $79 \%$.

${ }^{1} \mathrm{H}$ NMR (400 MHz, Chloroform-d) $\delta 8.09(\mathrm{~m}, 2 \mathrm{H}), 8.03-7.90(\mathrm{~m}, 1 \mathrm{H}), 7.74(\mathrm{~s}, 1 \mathrm{H})$, 7.69 (dd, J = 7.6, 2.0 Hz, 2H), 7.64 (dtt, J = 7.0, 5.0, 2.0 Hz, 2H), 7.52 (td, J = 7.6, 1.8 $\mathrm{Hz}, 2 \mathrm{H}), 7.44(\mathrm{dd}, \mathrm{J}=8.2,6.2 \mathrm{~Hz}, 1 \mathrm{H}) .{ }^{13} \mathrm{C}$ NMR $(101 \mathrm{MHz}$, Chloroform-d) $\delta 146.2$, $139.4,138.7,135.2,129.3,128.5,128.4,128.0,127.9,127.5,126.2,125.5,120.9$, 119.0 (q, J = 320.3 Hz), 117.8. ${ }^{19} \mathrm{~F}$ NMR (565 MHz, No Solvent) $\delta$-74.5.

HRMS (IT/TOF): calcd for $\mathrm{C}_{17} \mathrm{H}_{11} \mathrm{~F}_{3} \mathrm{O}_{3} \mathrm{~S}$ [M-H] 351.0308 (Negative mode), found: 351.0310 .

3-(p-tolyl)naphthalen-1-yl trifluoromethanesulfonate (2b):<smiles>CCOc1cc(-c2ccc(C)cc2)cc2ccccc12</smiles> 
Purification by flash chromatography on silica gel (petroleum ether) provided $2 \mathrm{~b}$ as a colorless oil; $\mathrm{R}_{f}=0.2$ (petroleum ether); $40 \mathrm{mg}$, isolated yield: $55 \%$.

${ }^{1} \mathrm{H}$ NMR (400 MHz, Chloroform-d) $\delta 8.13-8.06$ (m, 1H), 8.05 (s, 1H), 7.95 (m, 1H), $7.73(\mathrm{~s}, 1 \mathrm{H}), 7.68-7.55(\mathrm{~m}, 4 \mathrm{H}), 7.33(\mathrm{~d}, \mathrm{~J}=7.9 \mathrm{~Hz}, 2 \mathrm{H}), 2.45(\mathrm{~s}, 3 \mathrm{H}) .{ }^{13} \mathrm{C} \mathrm{NMR}$ (101 MHz, Chloroform-d) $\delta$ 146.2, 138.7, 138.3, 136.5, 135.2, 130.0, 128.4, 127.9, $127.7,127.3,125.8,125.3,120.9,119.0$ ( q, J = 320.2 Hz), 117.7, 21.3. ${ }^{19} \mathrm{~F}$ NMR (565 $\mathrm{MHz}$, No Solvent ) $\delta-74.5$.

HRMS(IT/TOF): calcd for $\mathrm{C}_{17} \mathrm{H}_{11} \mathrm{~F}_{3} \mathrm{O}_{3} \mathrm{~S}$ [M-H] $]^{-}: 365.0459$ (Negative mode), found: 365.0460 .

\section{3-(4-ethylphenyl)naphthalen-1-yl trifluoromethanesulfonate (2c):}<smiles>CCOc1cc(-c2ccc(CC)cc2)cc2ccccc12</smiles>

Purification by flash chromatography on silica gel (petroleum ether) provided $2 \mathrm{c}$ as a light yellow oil; $\mathrm{R}_{f}=0.2$ (petroleum ether); $43 \mathrm{mg}$, isolated yield: $57 \%$.

${ }^{1} \mathrm{H}$ NMR (400 MHz, Chloroform-d) $\delta 8.13-8.06(\mathrm{~m}, 1 \mathrm{H}), 8.05$ (s, 1H), $7.99-7.88$ (m, 1H), $7.73(\mathrm{~d}, \mathrm{~J}=1.6 \mathrm{~Hz}, 1 \mathrm{H}), 7.68-7.57(\mathrm{~m}, 4 \mathrm{H}), 7.36(\mathrm{~d}, \mathrm{~J}=8.2 \mathrm{~Hz}, 2 \mathrm{H}), 2.75$ (q, J = 7.5 Hz, 2H), 1.32 (t, J = 7.6 Hz, 3H). ${ }^{13} \mathrm{C}$ NMR (101 MHz, Chloroform-d) $\delta$ 146.2 , 144.7, 138.7, 136.7, 135.2, 128.8, 128.4, 127.9, 127.7, 127.4, 125.8, 125.3, $120.9,119.0$ (q, J = 320.3 Hz), 117.7, 28.7, 15.7. ${ }^{19} \mathrm{~F}$ NMR (565 MHz, No Solvent) $\delta$ -73.1 .

HRMS(IT/TOF): calcd for $\mathrm{C}_{17} \mathrm{H}_{11} \mathrm{~F}_{3} \mathrm{O}_{3} \mathrm{~S}$ [M-H] 379.0616 (Negative mode), found: 379.0616 .

\section{3-(4-(tert-butyl)phenyl)naphthalen-1-yl trifluoromethanesulfonate (2d):}<smiles>CCOc1cc(-c2ccc(C(C)(C)C)cc2)cc2ccccc12</smiles>

Purification by flash chromatography on silica gel (petroleum ether) provided $2 \mathrm{~d}$ as a light yellow oil; $\mathrm{R}_{f}=0.2$ (petroleum ether); $56 \mathrm{mg}$, isolated yield: $69 \%$.

${ }^{1} \mathrm{H}$ NMR (400 MHz, Chloroform-d) $\delta 8.14-8.08(\mathrm{~m}, 1 \mathrm{H}), 8.07$ (s, 1H), 7.96 (dd, J = 7.7, $1.7 \mathrm{~Hz}, 1 \mathrm{H}), 7.75(\mathrm{~d}, \mathrm{~J}=1.5 \mathrm{~Hz}, 1 \mathrm{H}), 7.68-7.59(\mathrm{~m}, 4 \mathrm{H}), 7.59-7.53(\mathrm{~m}, 2 \mathrm{H})$, 1.42 (s, 9H). ${ }^{13} \mathrm{C}$ NMR (101 MHz, Chloroform-d) $\delta 151.6,146.2,138.6,136.5,135.2$, 128.45, 127.9, 127.7, 127.1, 126.3, 125.9, 125.4, 120.9, 119.0 (q, J = 320.6 Hz), 117.7, 34.8, 31.5. ${ }^{19} \mathrm{~F}$ NMR (283 MHz, Chloroform-d) $\delta$-73.2.

HRMS(IT/TOF): calcd for $\mathrm{C}_{21} \mathrm{H}_{19} \mathrm{~F}_{3} \mathrm{O}_{3} \mathrm{~S}$ [M-H] $: 407.0929$ (Negative mode), found: 407.0931. 


\section{3-(4-fluorophenyl)naphthalen-1-yl trifluoromethanesulfonate (2f):}<smiles>CCOc1cc(-c2ccc(F)cc2)cc2ccccc12</smiles>

Purification by flash chromatography on silica gel (petroleum ether) provided $2 \mathrm{f}$ as a light yellow oil; $\mathrm{R}_{f}=0.2$ (petroleum ether); $56 \mathrm{mg}$, isolated yield: $75 \%$.

${ }^{1} \mathrm{H}$ NMR (400 MHz, Chloroform-d) $\delta 8.13-8.04(\mathrm{~m}, 1 \mathrm{H}), 8.00(\mathrm{~s}, 1 \mathrm{H}), 7.95$ (dd, J = 6.9, $2.2 \mathrm{~Hz}, 1 \mathrm{H}), 7.65(\mathrm{~m}, 5 \mathrm{H}), 7.25-7.14(\mathrm{~m}, 2 \mathrm{H}) .{ }^{13} \mathrm{C}$ NMR (101 MHz, Chloroform-d) $\delta 163.1(\mathrm{~d}, \mathrm{~J}=248.1 \mathrm{~Hz}), 146.2,137.7,135.5(\mathrm{~d}, \mathrm{~J}=3.3 \mathrm{~Hz}), 135.1$, 129.1(d, J = 7.9 Hz) 128.5, $128.0(\mathrm{~d}, \mathrm{~J}=8.0 \mathrm{~Hz}), 126.0,125.4,120.9,118.9$ (q, J = $320.5 \mathrm{~Hz}), 117.6,116.4,116.1 .{ }^{19} \mathrm{~F}$ NMR (283 MHz, Chloroform-d) $\delta$-74.3, -115.1.

HRMS(IT/TOF): calcd for $\mathrm{C}_{17} \mathrm{H}_{10} \mathrm{~F}_{4} \mathrm{O}_{3} \mathrm{~S}$ [M-H] $: 369.0209$ (Negative mode), found: 369.0210 .

\section{3-(4-bromophenyl)naphthalen-1-yl trifluoromethanesulfonate (2g):}<smiles>CCCc1cc(-c2ccc(Br)cc2)cc2ccccc12</smiles>

Purification by flash chromatography on silica gel (petroleum ether) provided $2 \mathrm{~g}$ as a yellow solid; $\mathrm{R}_{f}=0.2$ (petroleum ether); $78 \mathrm{mg}$, isolated yield: $91 \%$.

${ }^{1} \mathrm{H}$ NMR (400 MHz, Chloroform-d) $\delta 8.09$ (d, J = 7.7 Hz, 1H), 8.01 (s, 1H), 7.94 (dd, $\mathrm{J}=7.3,1.9 \mathrm{~Hz}, 1 \mathrm{H}), 7.69-7.60(\mathrm{~m}, 5 \mathrm{H}), 7.56-7.49(\mathrm{~m}, 2 \mathrm{H}) .{ }^{13} \mathrm{C}$ NMR $(101 \mathrm{MHz}$, Chloroform-d) $\delta 146.2,138.2,137.4,135.1,132.4,129.0,128.5,128.2,128.1,126.1$, 125.6, 122.8, 120.9, 118.9 (q, J = 321.3 Hz), 117.3. ${ }^{19} \mathrm{~F}$ NMR (565 MHz, No Solvent) $\delta-74.3$.

HRMS(IT/TOF): calcd for $\mathrm{C}_{17} \mathrm{H}_{10} \mathrm{BrF}_{3} \mathrm{O}_{3} \mathrm{~S}$ [M-H] $]^{-} 428.9408$ (Negative mode), found: 428.9411 .

\section{3-(4-chlorophenyl)naphthalen-1-yl trifluoromethanesulfonate (2h):}<smiles>CCOc1cc(-c2ccc(Cl)cc2)cc2ccccc12</smiles>

Purification by flash chromatography on silica gel (petroleum ether) provided $2 \mathrm{~h}$ as a yellow oil; $\mathrm{R}_{f}=0.2$ (petroleum ether); $69 \mathrm{mg}$, isolated yield: $90 \%$.

${ }^{1} \mathrm{H}$ NMR (400 MHz, Chloroform-d) $\delta 8.09$ (dd, J = 7.2, $\left.1.9 \mathrm{~Hz}, 1 \mathrm{H}\right), 8.01(\mathrm{~s}, 1 \mathrm{H})$, $7.95(\mathrm{dd}, \mathrm{J}=7.0,2.2 \mathrm{~Hz}, 1 \mathrm{H}), 7.69-7.56(\mathrm{~m}, 5 \mathrm{H}), 7.51-7.44(\mathrm{~m}, 2 \mathrm{H}) .{ }^{13} \mathrm{C} \mathrm{NMR}$ (101 MHz, Chloroform-d) $\delta$ 146.2, 137. 8, 137.4, 135.1, 134.6, 129.4, 128.7, 128.5, 
128.1, 126.1, 125.5, 120.9, 118.9 (q, J = 320.6 Hz), 117.4. ${ }^{19} \mathrm{~F}$ NMR (565 MHz, No Solvent) $\delta-74.6$.

HRMS(IT/TOF): calcd for $\mathrm{C}_{17} \mathrm{H}_{10} \mathrm{ClF}_{3} \mathrm{O}_{3} \mathrm{~S}$ [M-H] 384.9913 (Negative mode), found: 384.9915 .

\section{3-(2-chlorophenyl)naphthalen-1-yl trifluoromethanesulfonate (2i):}<smiles>CCOc1cc(-c2ccccc2Cl)cc2ccccc12</smiles>

Purification by flash chromatography on silica gel (petroleum ether) provided $2 \mathrm{i}$ as a light yellow oil; $\mathrm{R}_{f}=0.2$ (petroleum ether); $49 \mathrm{mg}$, isolated yield: $64 \%$.

${ }^{1} \mathrm{H}$ NMR (400 MHz, Chloroform-d) $\delta 8.13$ (d, $\left.J=8.4 \mathrm{~Hz}, 1 \mathrm{H}\right), 7.98-7.94(\mathrm{~m}, 1 \mathrm{H})$, $7.93(\mathrm{~s}, 1 \mathrm{H}), 7.73-7.61(\mathrm{~m}, 3 \mathrm{H}), 7.56-7.52(\mathrm{~m}, 1 \mathrm{H}), 7.45(\mathrm{~m}, 1 \mathrm{H}), 7.42-7.34(\mathrm{~m}$, $2 \mathrm{H}) .{ }^{13} \mathrm{C}$ NMR (101 MHz, Chloroform-d) $\delta 145.2,138.7,136.8,134.7,132.8,131.6$, $130.4,129.6,129.0,128.5,128.3,127.9,127.3,125.7,120.9,119.9 .118 .9$ (d, $J=$ $320.2 \mathrm{~Hz}) .{ }^{19} \mathrm{~F}$ NMR (283 MHz, Chloroform-d) $\delta$-74.4.

HRMS(IT/TOF): calcd for $\mathrm{C}_{17} \mathrm{H}_{10} \mathrm{ClF}_{3} \mathrm{O}_{3} \mathrm{~S}$ [M-H] 384.9913 (Negative mode), found: 384.9914 .

\section{3-(3-chlorophenyl)naphthalen-1-yl trifluoromethanesulfonate (2j):}<smiles>Oc1cc(-c2cccc(Cl)c2)cc2ccccc12</smiles>

Purification by flash chromatography on silica gel (petroleum ether) provided $2 \mathrm{j}$ as a light yellow oil; $\mathrm{R}_{f}=0.2$ (petroleum ether); $65 \mathrm{mg}$, isolated yield: $85 \%$.

${ }^{1} \mathrm{H}$ NMR (400 MHz, Chloroform-d) $\delta 8.10(\mathrm{dd}, \mathrm{J}=7.5,2.0 \mathrm{~Hz}, 1 \mathrm{H}), 8.04(\mathrm{~s}, 1 \mathrm{H})$, $7.96(\mathrm{dd}, \mathrm{J}=7.2,2.1 \mathrm{~Hz}, 1 \mathrm{H}), 7.66(\mathrm{~m}, 4 \mathrm{H}), 7.59-7.52(\mathrm{dt}, \mathrm{J}=7.5,1.9 \mathrm{~Hz}, 1 \mathrm{H}), 7.47$ $-7.38(\mathrm{~m}, 2 \mathrm{H}) .{ }^{13} \mathrm{C}$ NMR (101 MHz, Chloroform-d) $\delta$ 146. 2, 141.2, 137.2, 135.2, 135.1, 130.5, 128.6, 128.4, 128.3, 128.2, 127.6, 126.4, 125.7, 125.7, 120.9, 118.9 (q, J $=320.2 \mathrm{~Hz}), 117.4 .{ }^{19} \mathrm{~F}$ NMR (565 MHz, No Solvent) $\delta-74.3$.

HRMS(IT/TOF): calcd for $\mathrm{C}_{17} \mathrm{H}_{10} \mathrm{ClF}_{3} \mathrm{O}_{3} \mathrm{~S}$ [M-H] 384.9913 (Negative mode), found: 384.9914 . 


\section{3-(4-(trifluoromethyl)phenyl)naphthalen-1-yl trifluoromethanesulfonate (2k):}<smiles>CCOc1cc(-c2ccc(C(F)(F)F)cc2)cc2ccccc12</smiles>

Reaction at $80{ }^{\circ} \mathrm{C}$, purification by flash chromatography on silica gel (petroleum ether) provided $2 \mathrm{k}$ as a light yellow oil; $\mathrm{R}_{f}=0.2$ (petroleum ether); $33 \mathrm{mg}$, isolated yield: $40 \%$.

${ }^{1} \mathrm{H}$ NMR (400 MHz, Chloroform-d) $\delta 8.19-8.05$ (m, 2H), 7.99 (dd, J = 7.1, 2.0 Hz, 1H), $7.84-7.73(\mathrm{~m}, 4 \mathrm{H}), 7.73-7.61(\mathrm{~m}, 3 \mathrm{H}) .{ }^{13} \mathrm{C}$ NMR (101 MHz, Chloroform-d) $\delta$ 146.3, 142.9, 137.1, 135.1, 130.4 (q, J = 32.5 Hz), 128.7, 128.5, 128.3, 127.8, 126.8, 126.2 (q, J = 3.6 Hz), 125.9, 124.2 (q, J = 272.2 Hz), 121.0, $119.0(q, J=320.5 \mathrm{~Hz})$, 117.4. ${ }^{19}$ F NMR (565 MHz, No Solvent) $\delta-63.5,-74.7$.

HRMS(IT/TOF): calcd for $\mathrm{C}_{18} \mathrm{H}_{10} \mathrm{~F}_{6} \mathrm{O}_{3} \mathrm{~S}$ [M-H] $: 419.0177$ (Negative mode), found: 419.0179 .

methyl 4-((2-acetylphenyl)ethynyl)benzoate (2l'):<smiles>CC(=O)c1ccc(C#Cc2ccccc2C(C)=O)cc1</smiles>

Purification by flash chromatography on silica gel (petroleum ether/ethyl acetate) provided 2l' as a yellow solid; $\mathrm{R}_{f}=0.2$ (PE/EA $=20: 1$ ); $20 \mathrm{mg}$, isolated yield: $36 \%$.

${ }^{1} \mathrm{H}$ NMR (400 MHz, Chloroform-d) $\delta 8.07-7.98(\mathrm{~m}, 2 \mathrm{H}), 7.77(\mathrm{~d}, \mathrm{~J}=7.7 \mathrm{~Hz}, 1 \mathrm{H})$, $7.64(\mathrm{~d}, \mathrm{~J}=7.7 \mathrm{~Hz}, 1 \mathrm{H}), 7.63-7.57(\mathrm{~m}, 2 \mathrm{H}), 7.49$ (td, J=7.5, 1.7 Hz, 1H), 7.43 (t, J $=7.6 \mathrm{~Hz}, 1 \mathrm{H}), 3.93(\mathrm{~s}, 3 \mathrm{H}), 2.76(\mathrm{~s}, 3 \mathrm{H}) .{ }^{13} \mathrm{C} \mathrm{NMR}(101 \mathrm{MHz}$, Chloroform-d) $\delta 200.0$, 166.6, 140.8, 134.2, 131.6, 131.5, 130.0, 129.7, 129.0, 128.9, 127.7, 121. 2, 93.9, 91.4, $52.4,29.9$.

3-([1,1'-biphenyl]-4-yl)naphthalen-1-yl trifluoromethanesulfonate (2m):<smiles>CCCOc1cc(-c2ccc(-c3ccccc3)cc2)cc2ccccc12</smiles>

Purification by flash chromatography on silica gel (petroleum ether) provided $2 \mathrm{~m}$ as a white solid; $\mathrm{R}_{f}=0.2$ (petroleum ether); $51 \mathrm{mg}$, isolated yield: $60 \%$.

${ }^{1} \mathrm{H}$ NMR (400 MHz, Chloroform-d) $\delta 8.11(\mathrm{~m}, 2 \mathrm{H}), 8.02-7.94(\mathrm{~m}, 1 \mathrm{H}), 7.83-7.72$ $(\mathrm{m}, 5 \mathrm{H}), 7.72-7.60(\mathrm{~m}, 4 \mathrm{H}), 7.51(\mathrm{t}, \mathrm{J}=7.5 \mathrm{~Hz}, 2 \mathrm{H}), 7.41(\mathrm{t}, \mathrm{J}=7.4 \mathrm{~Hz}, 1 \mathrm{H}) .{ }^{13} \mathrm{C}$ NMR (101 MHz, Chloroform-d) $\delta 146.2,141.2,140.5,138.2,135.2,129.0,128.5$, 
$128.0,127.8,127.8,127.2,126.0,125.5,120.9,119.0(\mathrm{q}, \mathrm{J}=320.4 \mathrm{~Hz}), 117.6 .{ }^{19} \mathrm{~F}$ NMR (565 MHz, No Solvent) $\delta$-74.5.

HRMS(IT/TOF): calcd for $\mathrm{C}_{23} \mathrm{H}_{15} \mathrm{~F}_{3} \mathrm{O}_{3} \mathrm{~S}$ [M-H]-: 427.0616 (Negative mode), found: 427.0618 .

\section{3-(thiophen-3-yl)naphthalen-1-yl trifluoromethanesulfonate (2n):}<smiles>Brc1cc(-c2ccsc2)cc2ccccc12</smiles>

Purification by flash chromatography on silica gel (petroleum ether) provided $2 \mathrm{n}$ as a brownish oil; $\mathrm{R}_{f}=0.2$ (petroleum ether); $38 \mathrm{mg}$, isolated yield: $53 \%$.

${ }^{1} \mathrm{H}$ NMR (400 MHz, Chloroform-d) $\delta 8.10-8.00$ (m, 2H), $7.95-7.85$ (m, 1H), 7.72 $(\mathrm{d}, \mathrm{J}=1.5 \mathrm{~Hz}, 1 \mathrm{H}), 7.68-7.56(\mathrm{~m}, 3 \mathrm{H}), 7.52-7.43(\mathrm{~m}, 2 \mathrm{H}) .{ }^{13} \mathrm{C}$ NMR $(101 \mathrm{MHz}$, Chloroform-d) $\delta 146.1,140.6,135.2,133.3,128.3,128.0,127.8,127.2,126.2,125.4$, 125.1, 121.9, 120.9, 119.0 (q, J $=320.4 \mathrm{~Hz}), 117.2 .{ }^{19} \mathrm{~F}$ NMR (283 MHz, Chloroform- $d$ ) $\delta-74.4$.

HRMS(IT/TOF): calcd for $\mathrm{C}_{15} \mathrm{H}_{9} \mathrm{~F}_{3} \mathrm{O}_{3} \mathrm{~S}_{2}$ [M-H] 356.9867 (Negative mode), found: 356.9869 .

\section{6-fluoro-3-phenylnaphthalen-1-yl trifluoromethanesulfonate (2o):}<smiles>CCOc1cc(-c2ccccc2)cc2cc(F)ccc12</smiles>

Purification by flash chromatography on silica gel (petroleum ether) provided 2o as a light yellow oil; $\mathrm{R}_{f}=0.2$ (petroleum ether); $58 \mathrm{mg}$, isolated yield: $78 \%$.

${ }^{1} \mathrm{H}$ NMR (400 MHz, Chloroform-d) $\delta 8.09$ (dd, J = 9.3, $\left.5.2 \mathrm{~Hz}, 1 \mathrm{H}\right), 7.98(\mathrm{~s}, 1 \mathrm{H})$, $7.67(\mathrm{~m}, 3 \mathrm{H}), 7.60-7.49(\mathrm{~m}, 3 \mathrm{H}), 7.49-7.36(\mathrm{~m}, 2 \mathrm{H}) .{ }^{13} \mathrm{C}$ NMR (101 MHz, Chloroform-d) $\delta 161.8(\mathrm{~d}, \mathrm{~J}=249.9 \mathrm{~Hz}), 146.2,140.2,139.0,136.3(\mathrm{~d}, \mathrm{~J}=9.6 \mathrm{~Hz})$, 129.3, 128.6, 127.5, $125.4(\mathrm{~d}, \mathrm{~J}=5.1 \mathrm{~Hz}), 123.7(\mathrm{~d}, \mathrm{~J}=9.1 \mathrm{~Hz}), 122.5,118.9(\mathrm{~d}, J=$ $320.3 \mathrm{~Hz}), 118.3(\mathrm{~d}, \mathrm{~J}=25.8 \mathrm{~Hz}), 117.1,111.8(\mathrm{~d}, \mathrm{~J}=21.1 \mathrm{~Hz}) .{ }^{19} \mathrm{~F}$ NMR $(565 \mathrm{MHz}$, No Solvent) $\delta-74.2,-112.6$.

HRMS(IT/TOF): calcd for $\mathrm{C}_{17} \mathrm{H}_{10} \mathrm{~F}_{4} \mathrm{O}_{3} \mathrm{~S}$ [M-H] 369.0209 (Negative mode), found: 369.0210 . 
7-fluoro-3-phenylnaphthalen-1-yl trifluoromethanesulfonate (2p):<smiles>CCOc1cc(-c2ccccc2)cc2ccc(F)cc12</smiles>

Purification by flash chromatography on silica gel (petroleum ether) provided $2 \mathrm{p}$ as a light yellow solid; $\mathrm{R}_{f}=0.2$ (petroleum ether); $33 \mathrm{mg}$, isolated yield: $45 \%$.

${ }^{1} \mathrm{H}$ NMR (400 MHz, Chloroform-d) $\delta 8.05(\mathrm{~s}, 1 \mathrm{H}), 7.96(\mathrm{dd}, \mathrm{J}=9.1,5.4 \mathrm{~Hz}, 1 \mathrm{H})$, $7.77(\mathrm{~s}, 1 \mathrm{H}), 7.73-7.62(\mathrm{~m}, 3 \mathrm{H}), 7.58-7.49(\mathrm{~m}, 2 \mathrm{H}), 7.49-7.34(\mathrm{~m}, 2 \mathrm{H}) .{ }^{13} \mathrm{C} \mathrm{NMR}$ (101 MHz, Chloroform-d) $\delta 161.8(\mathrm{~d}, \mathrm{~J}=249.8 \mathrm{~Hz}), 145.5(\mathrm{~d}, \mathrm{~J}=5.7 \mathrm{~Hz}), 139.1$, 138.2, 132.2, 131.3 (d, J = 9.4 Hz), 129.3, 128.4, 127.4, 126.4 (d, J = 9.7 Hz), 126.1, 118.9 (q, J = 320.7 Hz), 118.9, $118.6(\mathrm{~d}, \mathrm{~J}=25.5 \mathrm{~Hz}), 105.1(\mathrm{~d}, \mathrm{~J}=23.9 \mathrm{~Hz}) .{ }^{19} \mathrm{~F}$ NMR (565 MHz, No Solvent) $\delta$-74.2, -111.0.

HRMS(IT/TOF): calcd for $\mathrm{C}_{17} \mathrm{H}_{10} \mathrm{~F}_{4} \mathrm{O}_{3} \mathrm{~S}$ [M-H]-: 369.0209 (Negative mode), found: 369.0211 .

\section{7-chloro-3-phenylnaphthalen-1-yl trifluoromethanesulfonate (2q):}<smiles>COc1cc(-c2ccccc2)cc2ccc(Cl)cc12</smiles>

Purification by flash chromatography on silica gel (petroleum ether) provided $2 \mathrm{q}$ as a light yellow oil; $\mathrm{R}_{f}=0.2$ (petroleum ether); $43 \mathrm{mg}$, isolated yield: $56 \%$.

${ }^{1} \mathrm{H}$ NMR (400 MHz, Chloroform-d) $\delta 8.07-8.01(\mathrm{~m}, 2 \mathrm{H}), 7.89(\mathrm{~d}, \mathrm{~J}=8.7 \mathrm{~Hz}, 1 \mathrm{H})$, $7.76(\mathrm{~d}, \mathrm{~J}=1.8 \mathrm{~Hz}, 1 \mathrm{H}), 7.74-7.64(\mathrm{~m}, 2 \mathrm{H}), 7.59-7.49(\mathrm{~m}, 3 \mathrm{H}), 7.47-7.41(\mathrm{~m}$, 1H). ${ }^{13} \mathrm{C}$ NMR (101 MHz, Chloroform-d) $\delta 145.1,139.1,139.0,134.2,133.4,130.1$, $129.4,129.1,128.6,127.4,126.1,125.9,120.0,118.9$ (q, J = 320.5 Hz), $118.8 .{ }^{19} \mathrm{~F}$ NMR (565 MHz, No Solvent) $\delta$-74.2.

HRMS(IT/TOF): calcd for $\mathrm{C}_{17} \mathrm{H}_{10} \mathrm{ClF}_{3} \mathrm{O}_{3} \mathrm{~S}$ [M-H] 384.9913 (Negative mode), found: 384.9915 .

\section{7-methyl-3-phenylnaphthalen-1-yl trifluoromethanesulfonate (2r):}<smiles>CCOc1cc(-c2ccccc2)cc2ccc(C)cc12</smiles>

Purification by flash chromatography on silica gel (petroleum ether) provided $2 \mathrm{r}$ as a light yellow solid; $\mathrm{R}_{f}=0.2$ (petroleum ether); $56 \mathrm{mg}$, isolated yield: $76 \%$.

${ }^{1} \mathrm{H}$ NMR (400 MHz, Chloroform-d) $\delta 8.01(\mathrm{~s}, 1 \mathrm{H}), 7.88-7.82(\mathrm{~m}, 2 \mathrm{H}), 7.69(\mathrm{~m}, 2 \mathrm{H})$, $7.67(\mathrm{~s}, 1 \mathrm{H}), 7.51(\mathrm{dd}, \mathrm{J}=8.3,7.0 \mathrm{~Hz}, 2 \mathrm{H}), 7.48-7.40(\mathrm{~m}, 2 \mathrm{H}), 2.60(\mathrm{~s}, 3 \mathrm{H}) .{ }^{13} \mathrm{C}$ NMR (101 MHz, Chloroform-d) $\delta 145.8,139.5,138.2,137.7,133.6,130.3,129.2$, 
128.4, 128.2, 127.4, 125.9, 125.7, 119.7, $119.0(\mathrm{q}, \mathrm{J}=320.4 \mathrm{~Hz}), 117.7,22.3 .{ }^{19} \mathrm{~F}$ NMR (565 MHz, No Solvent) $\delta$-74.7.,

HRMS(IT/TOF): calcd for $\mathrm{C}_{18} \mathrm{H}_{13} \mathrm{~F}_{3} \mathrm{O}_{3} \mathrm{~S}$ [M-H]': 365.0459 (Negative mode), found: 365.0460 .

\section{7-methoxy-3-phenylnaphthalen-1-yl trifluoromethanesulfonate (2s):}<smiles>CCOc1cc(-c2ccccc2)cc2ccc(OC)cc12</smiles>

Purification by flash chromatography on silica gel (petroleum ether) provided $2 \mathrm{~s}$ as a colorless oil; $\mathrm{R}_{f}=0.1$ (petroleum ether); $26 \mathrm{mg}$, isolated yield: $35 \%$.

${ }^{1} \mathrm{H}$ NMR (400 MHz, Chloroform-d) $\delta 7.99$ (s, 1H), 7.85 (d, J = 8.9 Hz, 1H), 7.70 (d, J $=1.7 \mathrm{~Hz}, 1 \mathrm{H}), 7.69-7.61(\mathrm{~m}, 2 \mathrm{H}), 7.54-7.46(\mathrm{~m}, 2 \mathrm{H}), 7.44-7.36(\mathrm{~m}, 1 \mathrm{H}), 7.32(\mathrm{~s}$, $\mathrm{J}=2.5 \mathrm{~Hz}, 1 \mathrm{H}), 7.27(\mathrm{~d}, \mathrm{~J}=7.1 \mathrm{~Hz}, 1 \mathrm{H}), 3.98(\mathrm{~s}, 3 \mathrm{H}) .{ }^{13} \mathrm{C} \mathrm{NMR}(101 \mathrm{MHz}$, Chloroform-d) $\delta 159.3,145.4,139.5,136.3,130.7,130.2,129.2,128.0,127.3,126.7$, 126.0, 121.1, 118.9 (q, J = 320.1 Hz), 118.2, 98.7, 55.6. ${ }^{19} \mathrm{~F}$ NMR (565 MHz, No Solvent) $\delta-74.4$.

HRMS(IT/TOF): calcd for $\mathrm{C}_{18} \mathrm{H}_{13} \mathrm{~F}_{3} \mathrm{O}_{4} \mathrm{~S}$ [M-H]': 381.0408 (Negative mode), found: 381.0410 . 


\subsection{To test other benzodiyne-type analogues under standard conditions}

To a solution of the appropriate benzodiyne $1(0.2 \mathrm{mmol})$ in freshly distilled $\mathrm{CHCl}_{3}$ $(10 \mathrm{~mL})$ under $\mathrm{N}_{2}$ atmosphere was added trifluoromethanesulfonic acid $(18 \mu \mathrm{L}, 0.2$ mmol) at room temperature. The resulting dark brown reaction mixture was then stirred at $50{ }^{\circ} \mathrm{C}$ until complete consumption of starting material was observed by TLC (For convenience, about $12 \mathrm{~h}$ ). On completion the reaction was quenched with water, extracted with EA. The organic layer was dried over $\mathrm{Na}_{2} \mathrm{SO}_{4}$, filtered, and concentrated. The crude residue was finally purified by column chromatography over silica gel using petroleum ether/ethyl acetate as eluent to yield the desired title compound 2 . The results were shown below.<smiles>C(#Cc1ccccc1C#Cc1ccccc1)c1ccccc1</smiles>

$1 \mathrm{u}$<smiles>[R]C#Cc1ccccc1C#CBr</smiles>

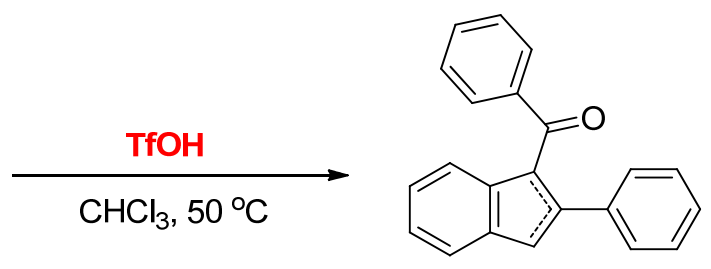

$$
2 \mathbf{u}^{\prime}(35 \%) \text { : mixture of }
$$
isomers

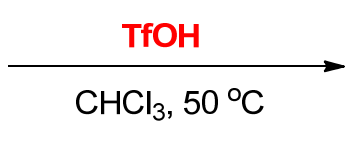

$$
2 v^{\prime}+2 w^{\prime}
$$

$2 \mathbf{v}^{\prime}\left(\mathrm{R}={ }^{\mathrm{n}} \mathrm{Bu}\right)$ : unidentified oligomer

2w' $(R=H)$ : unidentified addition mixture between

$1 \mathbf{w}$ and $\mathrm{TfOH}$, detected by GC-MASS

phenyl(2-phenyl-1H-inden-3-yl)methanone (2u')<smiles></smiles>

Purification by flash chromatography on silica gel (petroleum ether/ethyl acetate) provided $2 \mathrm{u}$ ' as a yellow oil; $\mathrm{R}_{f}=0.3(\mathrm{PE} / \mathrm{EA}=20: 1) ; 20 \mathrm{mg}$, isolated yield: $35 \%$. ${ }^{1} \mathrm{H}$ NMR (400 MHz, Chloroform-d) $\delta 7.97-7.90(\mathrm{~m}, 2 \mathrm{H}), 7.60-7.44(\mathrm{~m}, 3 \mathrm{H}), 7.41$ $-7.37(\mathrm{~m}, 2 \mathrm{H}), 7.34(\mathrm{t}, \mathrm{J}=7.7 \mathrm{~Hz}, 3 \mathrm{H}), 7.28(\mathrm{~m}, 3 \mathrm{H}), 7.25-7.18(\mathrm{~m}, 3 \mathrm{H}), 4.06(\mathrm{~s}$, 2H). ${ }^{13} \mathrm{C}$ NMR (101 MHz, Chloroform-d) $\delta 196.7,146.9,144.7,142.0,138.4,136.7$, $135.2,133.7,129.8,128.6,128.6,128.3,128.2,127.0,125.8,123.9,121.1,41.4$. 


\subsection{Scale synthesis for the preparation of naphthalenyl triflates.}

To a solution of benzodiyne 1a $(10 \mathrm{mmol})$ in freshly distilled $\mathrm{CHCl}_{3}(60 \mathrm{~mL})$ under $\mathrm{N}_{2}$ atmosphere was added trifluoromethanesulfonic acid (885 $\left.\mu \mathrm{L}, 10 \mathrm{mmol}\right)$ at room temperature. The resulting dark brown reaction mixture was then stirred at $50{ }^{\circ} \mathrm{C}$ until complete consumption of starting material was observed by TLC (For convenience, about $12 \mathrm{~h}$ ). On completion the reaction was quenched with water, extracted with EA. The organic layer was dried over $\mathrm{Na}_{2} \mathrm{SO}_{4}$, filtered, and concentrated. The crude residue was finally purified by column chromatography over silica gel using petroleum ether as eluent to yield the desired title compound 2a $1.96 \mathrm{~g}$, in $56 \%$ yield.<smiles>C#Cc1ccccc1C#Cc1ccccc1</smiles>

$1 \mathrm{a}$

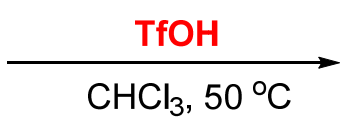

$10 \mathrm{mmol}$<smiles>CCCOc1cc(-c2ccccc2)cc2ccccc12</smiles>

2a: $1.96 \mathrm{~g}, 56 \%$ 


\section{Mechanistic study}

\subsection{To Test whether the Pd catalyzed the reaction.}

To a solution of the appropriate benzodiyne 1a $(0.2 \mathrm{mmol})$ in freshly distilled $\mathrm{CHCl}_{3}(10 \mathrm{~mL})$ under $\mathrm{N}_{2}$ atmosphere was added $\mathrm{Pd}(\mathrm{OAc})_{2}(2.3 \mathrm{mg}, 0.01 \mathrm{mmol})$ at room temperature. The reaction mixture was then stirred at $50{ }^{\circ} \mathrm{C}$ for $12 \mathrm{~h}$. The system was detected by GC-MASS after cooling to room temperature. Subsequently it was added trifluoromethanesulfonic acid $(18 \mu \mathrm{L}, 0.2 \mathrm{mmol})$. The resulting dark brown reaction mixture was then stirred at $50{ }^{\circ} \mathrm{C}$ for $12 \mathrm{~h}$. The system was detected by GC-MASS after cooling to room temperature (Eq 1).<smiles>C#Cc1ccccc1C#Cc1ccccc1</smiles>

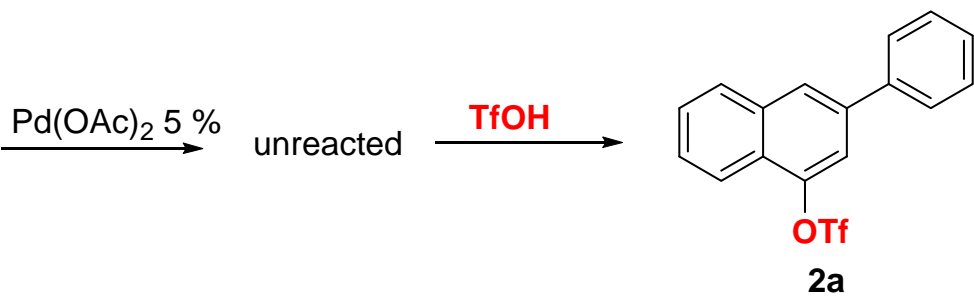

\subsection{Competitive reactions between OTf anion and solvent.}

To a solution of the appropriate benzodiyne $1 \mathrm{t}(0.2 \mathrm{mmol})$ in freshly distilled $\mathrm{CHCl}_{3}$ $(10 \mathrm{~mL})$ under $\mathrm{N}_{2}$ atmosphere was added trifluoromethanesulfonic acid $(18 \mu \mathrm{L}, 0.2$ mmol) at room temperature. The resulting dark brown reaction mixture was then stirred at $50{ }^{\circ} \mathrm{C}$ until complete consumption of starting material was observed by TLC (For convenience, about $12 \mathrm{~h}$ ). On completion the reaction was quenched with water, extracted with EA. The organic layer was dried over $\mathrm{Na}_{2} \mathrm{SO}_{4}$, filtered, and concentrated. The crude residue was finally purified by column chromatography over silica gel using petroleum ether as eluent to yield the desired title compound $2 \mathrm{t}^{\prime}, \mathrm{R}_{f}=$ 0.2 (petroleum ether) (Eq 2).

${ }^{1} \mathrm{H}$ NMR (400 MHz, Chloroform- $d$ ) $\delta 8.29-8.22(\mathrm{~m}, 1 \mathrm{H}), 7.95-7.85$ (m, 2H), 7.84 $(\mathrm{d}, J=1.7 \mathrm{~Hz}, 1 \mathrm{H}), 7.68-7.60(\mathrm{~m}, 2 \mathrm{H}), 7.56(\mathrm{tt}, J=6.9,5.2 \mathrm{~Hz}, 2 \mathrm{H}), 7.08-6.95(\mathrm{~m}$, 2H), 3.88 (s, 3H). ${ }^{13} \mathrm{C}$ NMR (101 MHz, Chloroform-d) $\delta 159.7,138.5,134.9,132.5$, $132.4,129.7,128.5,127.2,126.9,125.7,124.4,124.2,114.6,55.5$.

To a solution of the appropriate benzodiyne $1 \mathrm{a}(0.2 \mathrm{mmol})$ in freshly distilled benzene $(10 \mathrm{~mL})$ under $\mathrm{N}_{2}$ atmosphere was added trifluoromethanesulfonic acid $(18 \mu \mathrm{L}, 0.2$ mmol) at room temperature. The resulting dark brown reaction mixture was then stirred at $50{ }^{\circ} \mathrm{C}$ until complete consumption of starting material was observed by TLC (For convenience, about $12 \mathrm{~h}$ ). On completion the reaction was quenched with water, extracted with EA. The organic layer was dried over $\mathrm{Na}_{2} \mathrm{SO}_{4}$, filtered, and concentrated. The crude residue was finally purified by column chromatography over silica gel using petroleum ether/ethyl acetate as eluent to yield the desired title compound 2a', $\mathrm{R}_{f}=0.3$ (petroleum ether) (Eq 3 ). 


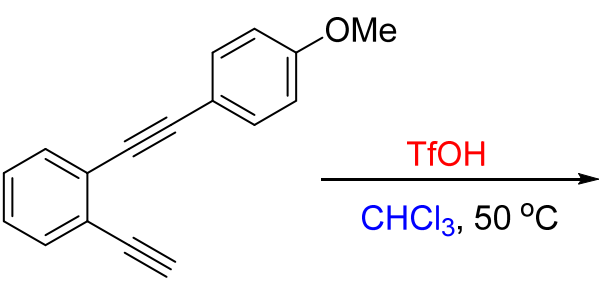

$1 \mathrm{t}$<smiles>C#Cc1ccccc1C#Cc1ccccc1</smiles>

$1 \mathrm{a}$<smiles>CCCOc1cc(-c2ccc(OC)cc2)cc2ccccc12</smiles>

2t' (42\%)

$2 t$ trace<smiles>c1ccc(-c2cc(-c3ccccc3)c3ccccc3c2)cc1</smiles>

2a' (10\%)<smiles>CCOc1cc(-c2ccccc2)cc2ccccc12</smiles>

2a trace 


\section{Derivatization of naphthalenyl Triflates. 1-methyl-3-phenylnaphthalene (4): ${ }^{2}$}<smiles>Cc1cc(-c2ccccc2)cc2ccccc12</smiles>

A round bottom flask containing 3-phenylnaphthalen-1-yl trifluoromethanesulfonate (70.5 mg, $0.2 \mathrm{mmol})$ and $\left[\mathrm{Ni}(\mathrm{dppp}) \mathrm{Cl}_{2}\right](5.4 \mathrm{mg}, 0.01 \mathrm{mmol})$ was evacuated and refilled three times with $\mathrm{N}_{2}$. After addition of $\mathrm{Et}_{2} \mathrm{O}(1.5 \mathrm{~mL})$, the solution was purged with $\mathrm{N}_{2}$ for 10 minutes, cooled to $0{ }^{\circ} \mathrm{C}$, treated with $\mathrm{MeMgBr}$ in THF $(0.2 \mathrm{ml}, 0.6$ mmol) dropwise, warmed to $40{ }^{\circ} \mathrm{C}$, and stirred for $16 \mathrm{~h}$. The mixture was cooled to $0{ }^{\circ} \mathrm{C}$ and treated dropwise with $\mathrm{H}_{2} \mathrm{O}(1 \mathrm{~mL})$. After the addition of $1 \mathrm{M} \mathrm{HCl}(2 \mathrm{~mL})$ the aqueous phase was extracted with $\mathrm{Et}_{2} \mathrm{O}(3 \times 10 \mathrm{~mL})$. The combined organic layers were washed with saturated aqueous $\mathrm{NaHCO}_{3}$ solution, dried over $\mathrm{Na}_{2} \mathrm{SO}_{4}$, and filtered through $\mathrm{SiO}_{2}$. Solvents were removed under reduced pressure and the crude product was purified by flash chromatography on silica gel to give the title compound as a colorless oil, $\mathrm{R}_{f}=0.3$ (petroleum ether); $36 \mathrm{mg}$, isolated yield: $82 \%$.

${ }^{1} \mathrm{H}$ NMR (400 MHz, Chloroform-d) $\delta 8.11-8.00(\mathrm{~m}, 1 \mathrm{H}), 7.97-7.88(\mathrm{~m}, 2 \mathrm{H}), 7.81$ - $7.70(\mathrm{~m}, 2 \mathrm{H}), 7.64(\mathrm{~s}, 1 \mathrm{H}), 7.60-7.48(\mathrm{~m}, 4 \mathrm{H}), 7.41(\mathrm{t}, \mathrm{J}=7.4 \mathrm{~Hz}, 1 \mathrm{H}), 2.80$ (s, $3 \mathrm{H}) .{ }^{13} \mathrm{C}$ NMR (101 MHz, Chloroform-d) $\delta$ 141.3, 138.3, 135.0, 134.0, 132.0, 129.0, $128.9,127.5,127.4,126.5,126.1,126.0,124.4,124.1,19.7$. Spectral data of 4 were consistent with data reported in the literature. ${ }^{3}$

\section{1-phenyl-1,2'-binaphthalene (5): ${ }^{4}$}<smiles>c1ccc(-c2cc(-c3ccc4ccccc4c3)c3ccccc3c2)cc1</smiles>

A mixture of 2-naphthaleneboronic acid (37.8 $\mathrm{mg}, \quad 0.22 \mathrm{mmol})$, 3-phenylnaphthalen-1-yl trifluoromethanesulfonate (70.4 mg, $0.2 \mathrm{mmol}), \mathrm{Pd}\left(\mathrm{PPh}_{3}\right)_{4}$ $(11.56 \mathrm{mg}, 0.01 \mathrm{mmol})$, and $\mathrm{K}_{3} \mathrm{PO}_{4}(63.7 \mathrm{mg}, 0.3 \mathrm{mmol})$ in dioxane $(1 \mathrm{~mL})$ was heated to $85{ }^{\circ} \mathrm{C}$ for $16 \mathrm{~h}$. The product was extracted with EA, washed with brine, and dried over $\mathrm{Na}_{2} \mathrm{SO}_{4}$. Isolation by column chromatography over silica gel gave following compounds as a light yellow solid, $\mathrm{R}_{f}=0.3$ (petroleum ether); $53 \mathrm{mg}$, isolated yield: $81 \%$. 
${ }^{1} \mathrm{H}$ NMR (400 MHz, Chloroform-d) $\delta 8.14(\mathrm{~d}, \mathrm{~J}=1.7 \mathrm{~Hz}, 1 \mathrm{H}), 8.06(\mathrm{~d}, \mathrm{~J}=1.6 \mathrm{~Hz}$, $1 \mathrm{H}), 8.04-7.92(\mathrm{~m}, 5 \mathrm{H}), 7.86(\mathrm{~d}, \mathrm{~J}=1.9 \mathrm{~Hz}, 1 \mathrm{H}), 7.84-7.79(\mathrm{~m}, 2 \mathrm{H}), 7.73(\mathrm{dd}, \mathrm{J}=$ 8.3, $1.8 \mathrm{~Hz}, 1 \mathrm{H}), 7.64-7.49(\mathrm{~m}, 5 \mathrm{H}), 7.50-7.38(\mathrm{~m}, 2 \mathrm{H}) .{ }^{13} \mathrm{C}$ NMR (101 MHz, Chloroform-d) $\delta 141.0,140.9,138.3,138.2,134.3,133.5,132.7,131.1,129.0,128.9$, $128.8,128.6,128.2,127.9,127.9,127.6,127.1,126.5,126.4,126.3,126.2,126.1$, 125.7 .

\section{1,3-diphenylnaphthalene (6): ${ }^{5}$}<smiles>c1ccc(-c2cc(-c3ccccc3)c3ccccc3c2)cc1</smiles>

A 25-mL, one-necked, round-bottomed flask equipped with a rubber septum was charged with 3-phenylnaphthalen-1-yl trifluoromethanesulfonate 2a (105.7 mg, 0.3 mmol) and tributylphenyltin (121.1 $\mathrm{mg}, 0.33 \mathrm{mmol})$ in $1 \mathrm{~mL}$ of DMF. Bis(triphenylphosphine)palladium(II) chloride (10.5 mg, $0.015 \mathrm{mmol}$ ) was added and the resulting yellow suspension was stirred at room temperature for $30 \mathrm{~min}$. The reaction mixture was then heated at $60{ }^{\circ} \mathrm{C}$ for an additional $8 \mathrm{~h}$. The reaction mixture was allowed to cool and then filtered through silica gel in a short column with the aid of $\mathrm{Et}_{2} \mathrm{O}$ to remove the majority of the residual palladium. The filtrate was then washed with two 2-mL portions of $1 \mathrm{~N}$ aq $\mathrm{HCl}$ solution, five 2-mL portions of water, and two 2-mL portions of saturated $\mathrm{NaCl}$ solution, dried over $\mathrm{Na}_{2} \mathrm{SO}_{4}$, filtered, and concentrated to afford a dark brown oil. Column chromatography silica gel (elution with petroleum ether) furnished the desired compound as a colorless oil, $\mathrm{R}_{f}=0.3$, (petroleum ether), $63 \mathrm{mg}$, isolated yield: $75 \%$.

${ }^{1} \mathrm{H}$ NMR (400 MHz, Chloroform-d) $\delta 8.11(\mathrm{~s}, 1 \mathrm{H}), 8.00$ (d, J = 8.1 Hz, 1H), 7.96 (d, J $=8.3 \mathrm{~Hz}, 1 \mathrm{H}), 7.80(\mathrm{~d}, \mathrm{~J}=7.4 \mathrm{~Hz}, 2 \mathrm{H}), 7.77(\mathrm{~d}, \mathrm{~J}=1.8 \mathrm{~Hz}, 1 \mathrm{H}), 7.65-7.38(\mathrm{~m}, 10 \mathrm{H})$.

${ }^{13} \mathrm{C}$ NMR (101 MHz, Chloroform-d) $\delta 141.0,141.0,140.8,138.1,134.3,131.0,130.2$, $129.0,128.7,128.5,127.6,127.5,126.8,126.4,126.2,126.0,125.5$. Spectral data of 4 were consistent with data reported in the literature. ${ }^{6}$ 


\section{3-phenyl-1-(p-tolylethynyl)naphthalene (7): ${ }^{7}$}<smiles>Cc1ccc(C#Cc2cc(-c3ccccc3)cc3ccccc23)cc1</smiles>

A mixture of 3-phenylnaphthalen-1-yl trifluoromethanesulfonate (70.5 mg, $0.2 \mathrm{mmol})$, 4-ethynyltoluene $(34.9 \mathrm{mg}, 0.3 \mathrm{mmol})$, triethylamine $(0.12 \mathrm{ml})$ and bis(triphenylphosphine)palladium(II) chloride $(7.0 \mathrm{mg}, 0.01 \mathrm{mmol})$ in $0.6 \mathrm{ml} \mathrm{DMF}$ was stirred at $90^{\circ} \mathrm{C}$ for $4 \mathrm{~h}$ under nitrogen. The reaction mixture was then diluted with water, extracted with EA, washed with water until neutral, dried by $\mathrm{Na}_{2} \mathrm{SO}_{4}$, then evaporated. Chromatography of the residue on silica gel (petroleum ether as eluate) provided the title compound as a colorless oil; $\mathrm{R}_{f}=0.3$ (petroleum ether); $59 \mathrm{mg}$, isolated yield: $94 \%$.

${ }^{1} \mathrm{H}$ NMR (400 MHz, Chloroform-d) $\delta 8.47(\mathrm{~d}, \mathrm{~J}=7.9 \mathrm{~Hz}, 1 \mathrm{H}), 8.08-8.02(\mathrm{~m}, 2 \mathrm{H})$, $7.98-7.86(\mathrm{~m}, 1 \mathrm{H}), 7.80-7.72(\mathrm{~m}, 2 \mathrm{H}), 7.63-7.54(\mathrm{~m}, 4 \mathrm{H}), 7.51(\mathrm{t}, \mathrm{J}=7.6 \mathrm{~Hz}, 2 \mathrm{H})$, $7.41(\mathrm{t}, \mathrm{J}=7.4 \mathrm{~Hz}, 1 \mathrm{H}), 7.23(\mathrm{~d}, \mathrm{~J}=7.8 \mathrm{~Hz}, 2 \mathrm{H}), 2.42(\mathrm{~s}, 3 \mathrm{H}) .{ }^{13} \mathrm{C}$ NMR $(101 \mathrm{MHz}$, Chloroform-d) $\delta 140.5,138.8,138.3,133.7,132.6,131.8,130.0,129.4,129.1,128.7$, $127.7,127.5,126.9,126.9,126.5,126.3,121.8,120.4,94.7,87.0,21.7$.

\section{References}

1 Byers, P. M.; Rashid, J. I.; Mohamed, R. K.; Alabugin, I. V. Org. Lett. 2012, 14 (23), 6032.

2 Schaack, C.; Sidler, S.; Trapp, N.; Diederich, F. Chem.-Eur. J. 2017, 23, 14153.

3 Mondal, S.; Gold, B.; Mohamed, R. K.; Phan, H.; Alabugin, I. V. J. Org. Chem. 2014, 79, 7491.

4 Ohe, T.; Miyaura, N.; Suzuki, A. J. Org. Chem. 1993, 58, 2201.

5 Dalton, A. M.; Zhang, Y. J.; Davie, C. P. Org. Lett. 2002, 4, 2465.

6 Jagdale, A. R.; Park, J. H.; Youn, S. W. J. Org. Chem. 2011, 76, 7204.

7 Chen, Q. Y.; Yang, Z. Y. Tetrahedron Lett. 1986, 27, 1171. 


\section{NMR spectra}

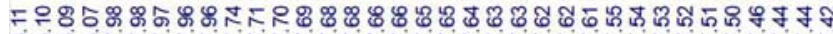 \\ क}

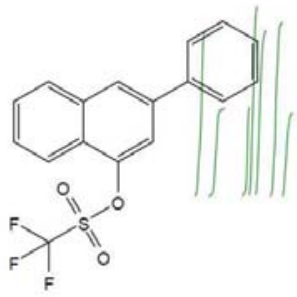

$2 a$

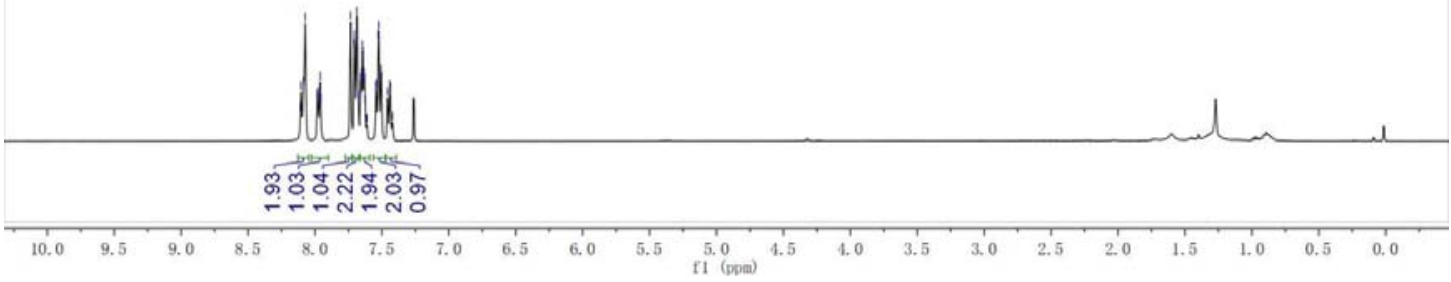

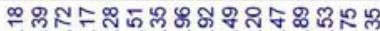

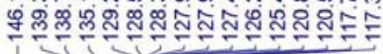

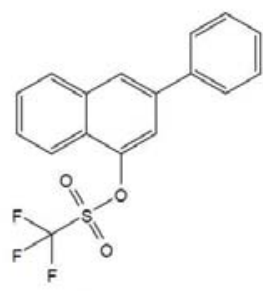

2a

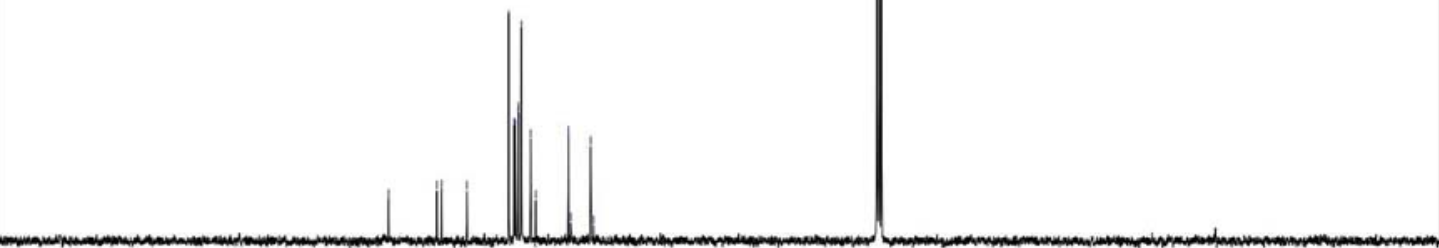

${ }^{1} \mathrm{H}$ NMR (400 MHz, $\mathrm{CDCl}_{3}$ ) (up) and ${ }^{13} \mathrm{C} \mathrm{NMR}\left(101 \mathrm{MHz}, \mathrm{CDCl}_{3}\right)$ (down) 


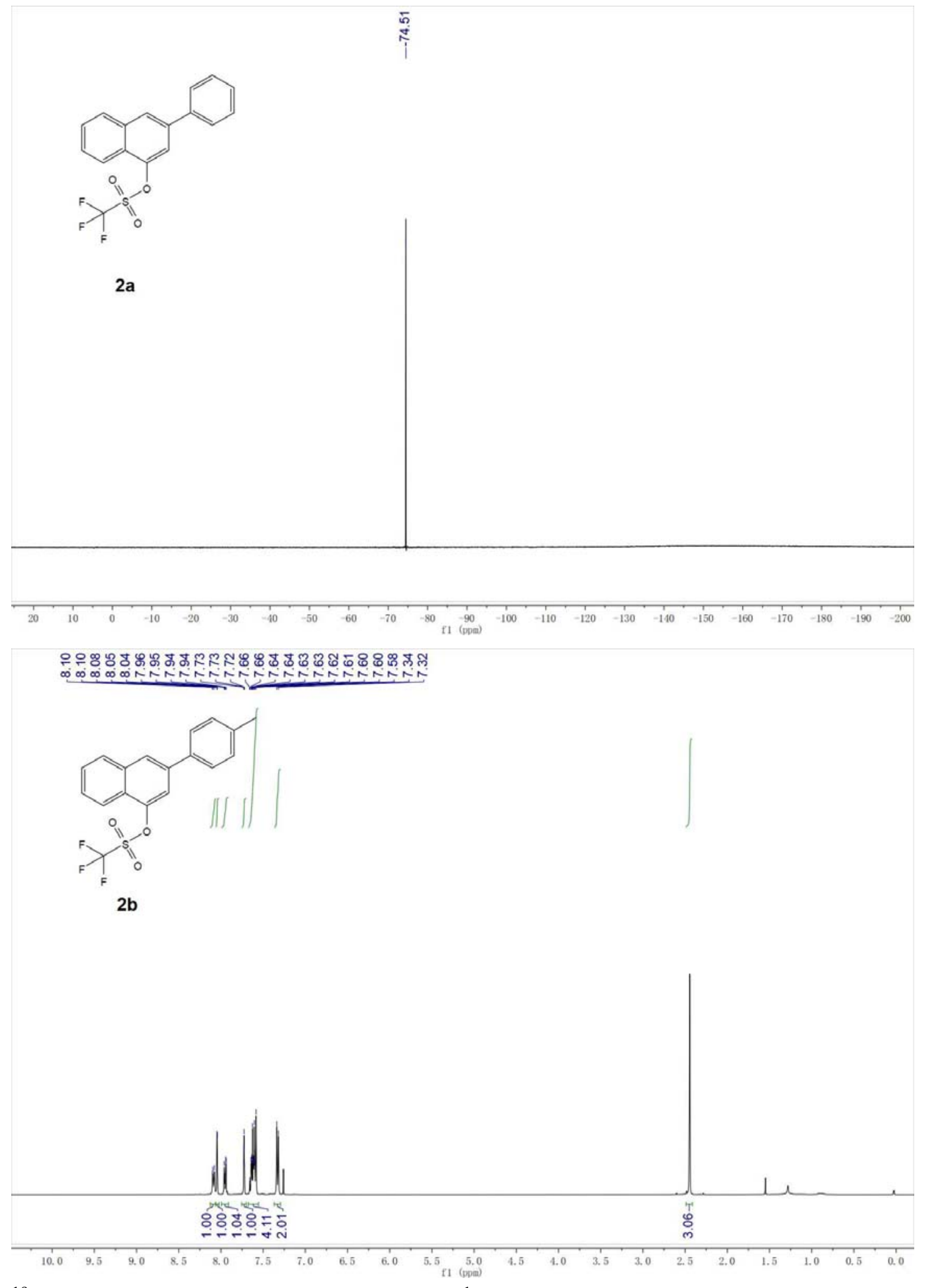

${ }^{19}$ F NMR (565 MHz, No Solvent) (up) and ${ }^{1} \mathrm{H}$ NMR (400 MHz, CDCl 3 ) (down) 

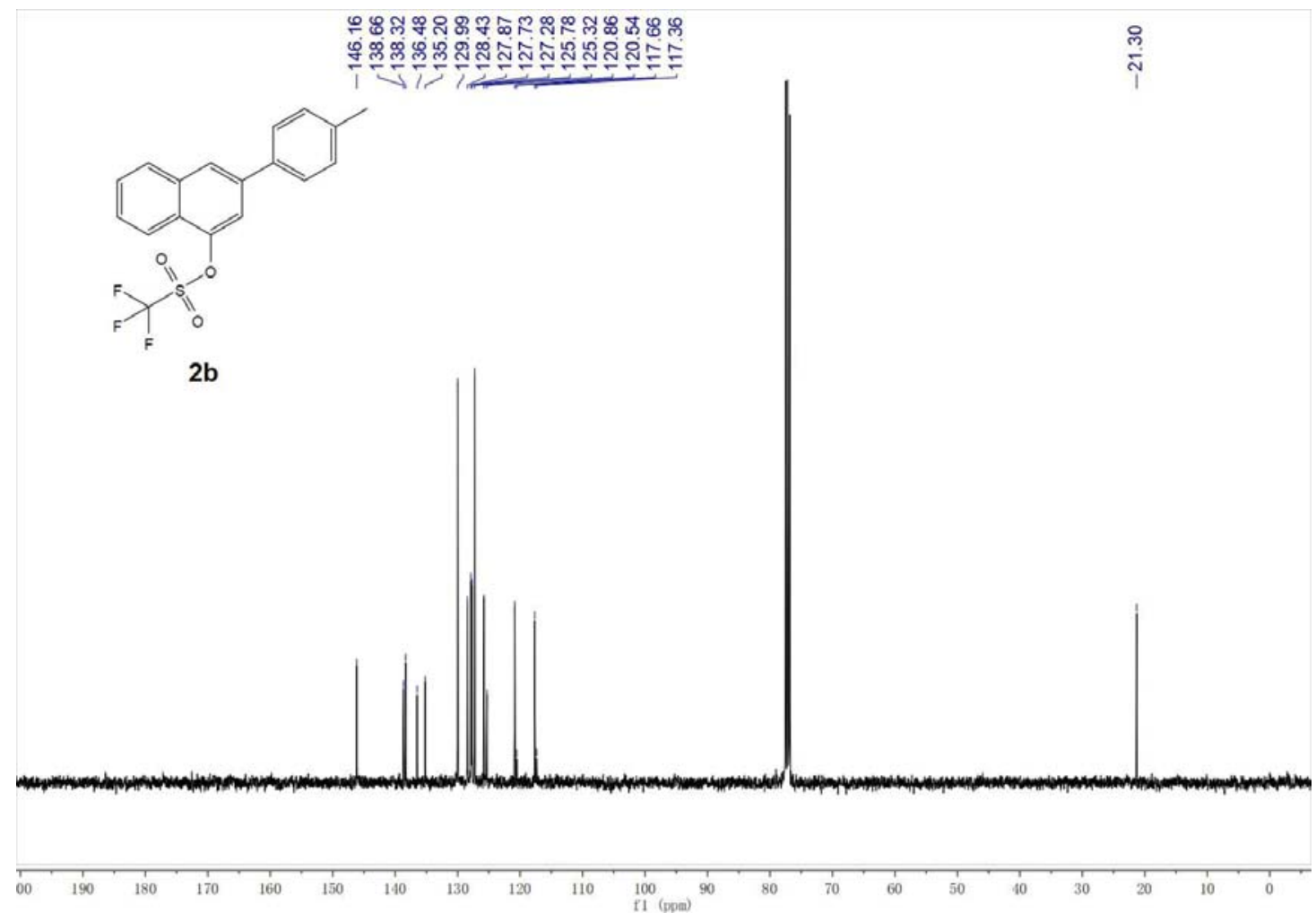

莡

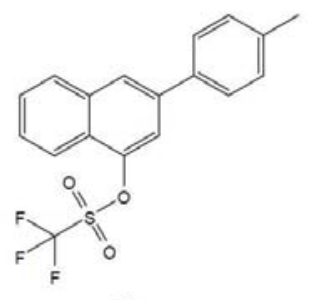

$2 b$
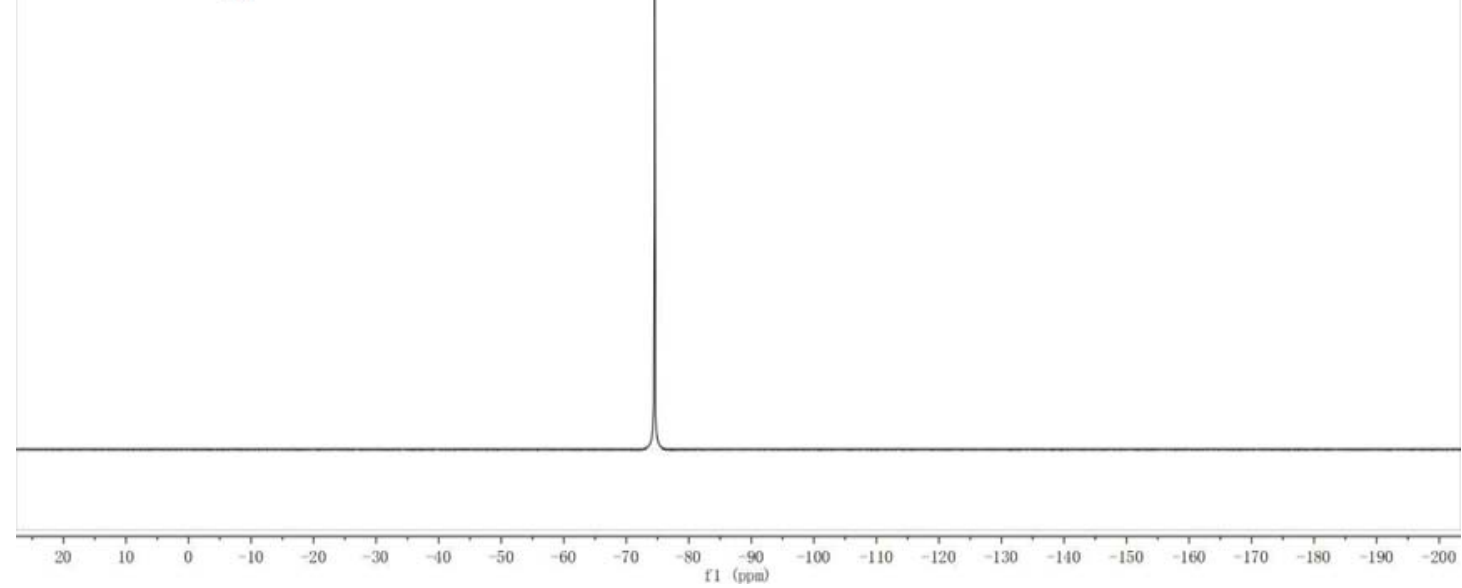

${ }^{13} \mathrm{C}$ NMR (101 MHz, CDCl 3 ) (up) and ${ }^{19} \mathrm{~F}$ NMR (565 MHz, No Solvent) (down) 


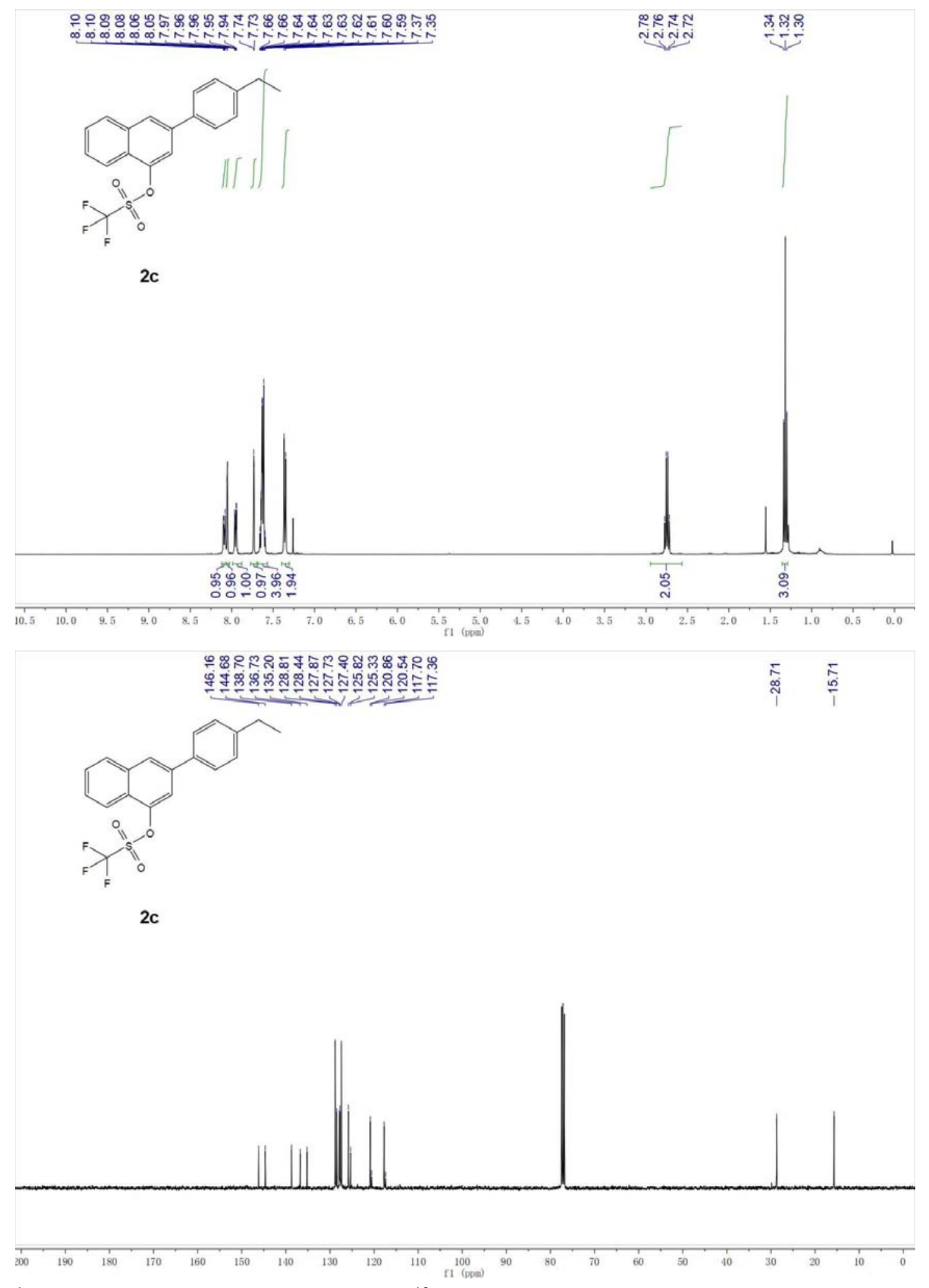

${ }^{1} \mathrm{H} \mathrm{NMR}\left(400 \mathrm{MHz}, \mathrm{CDCl}_{3}\right.$ ) (up) and ${ }^{13} \mathrm{C} \mathrm{NMR}$ (101 MHz, $\mathrm{CDCl}_{3}$ ) (down) 

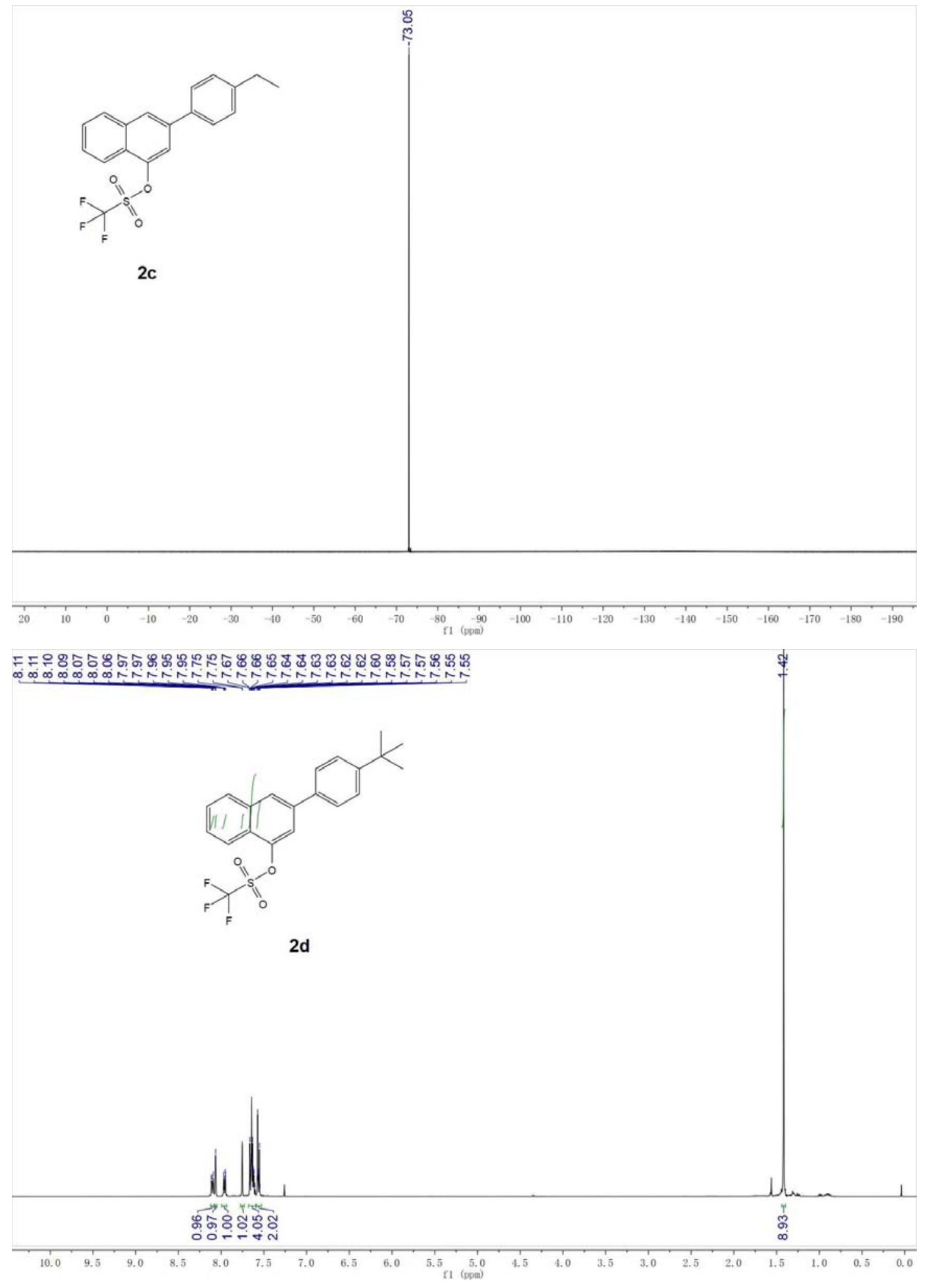

${ }^{19}$ F NMR (565 MHz, No Solvent) (up) and ${ }^{1} \mathrm{H}$ NMR (400 MHz, $\mathrm{CDCl}_{3}$ ) (down) 

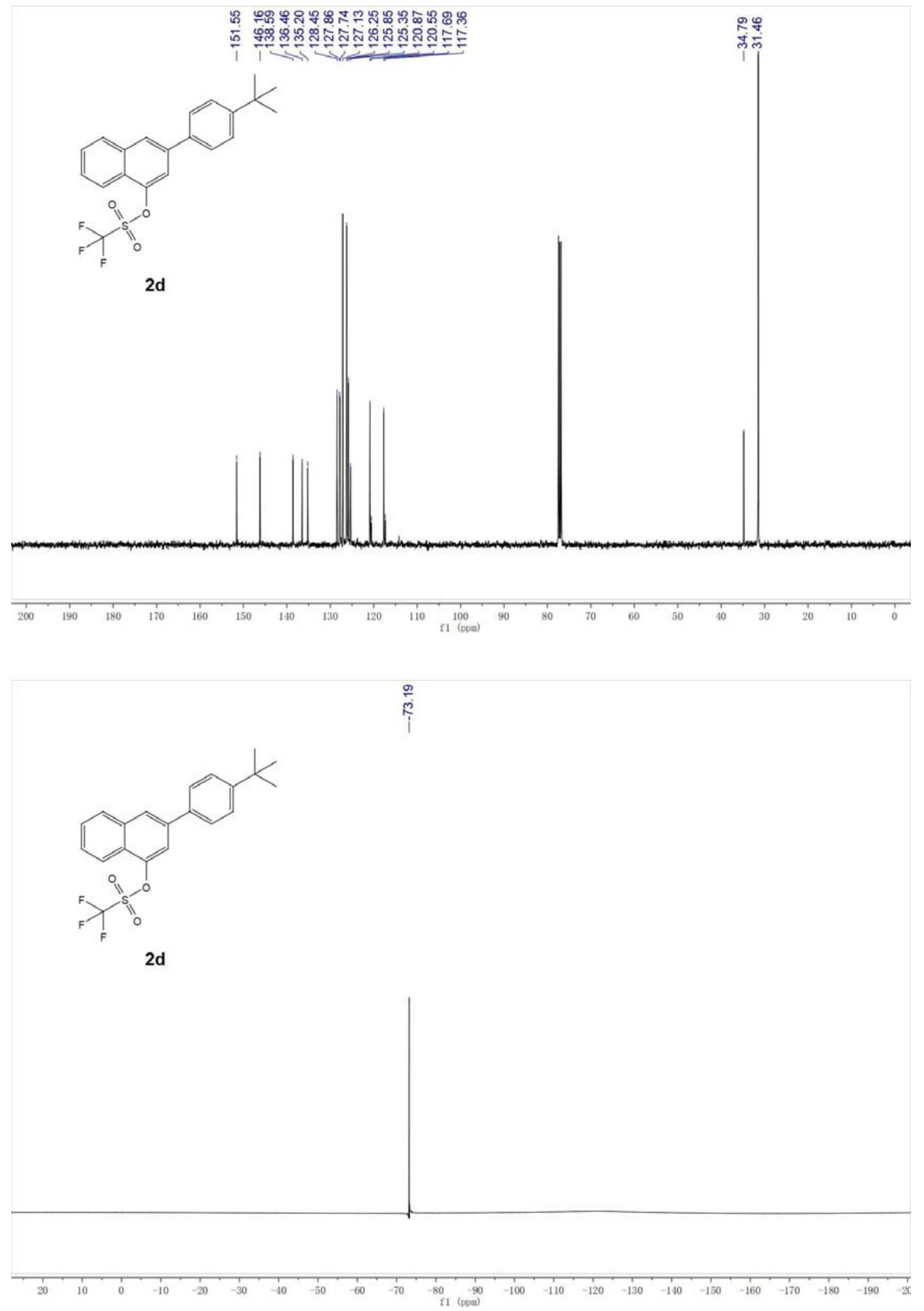

${ }^{13} \mathrm{C}$ NMR (101 MHz, CDCl 3 ) (up) and ${ }^{19} \mathrm{~F}$ NMR (283 MHz, $\mathrm{CDCl}_{3}$ ) (down) 


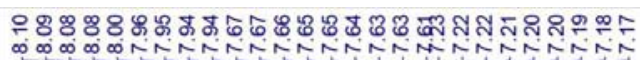
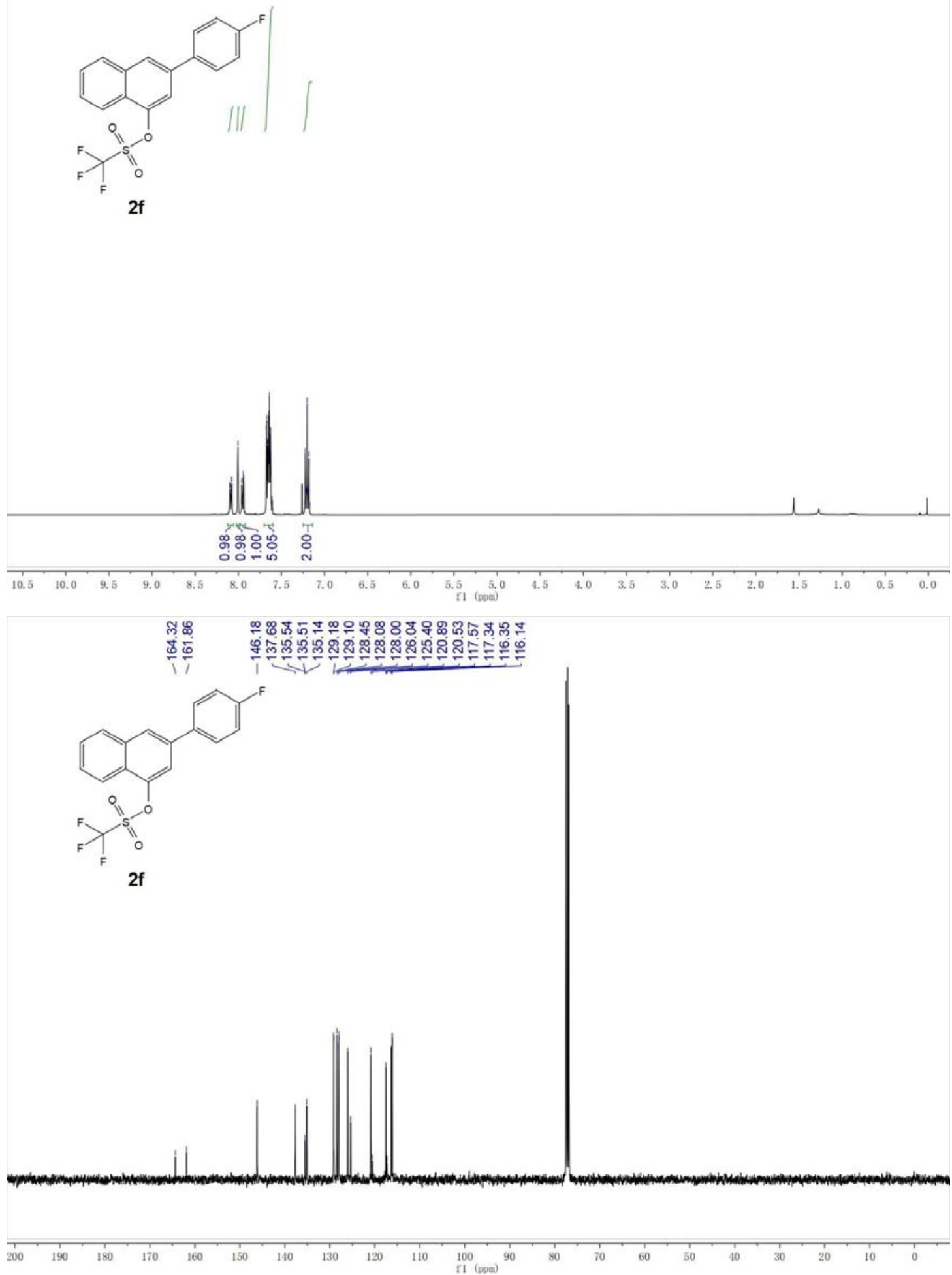

${ }^{1} \mathrm{H}$ NMR (400 MHz, $\mathrm{CDCl}_{3}$ ) (up) and ${ }^{13} \mathrm{C} \mathrm{NMR}\left(101 \mathrm{MHz}, \mathrm{CDCl}_{3}\right)$ (down) 


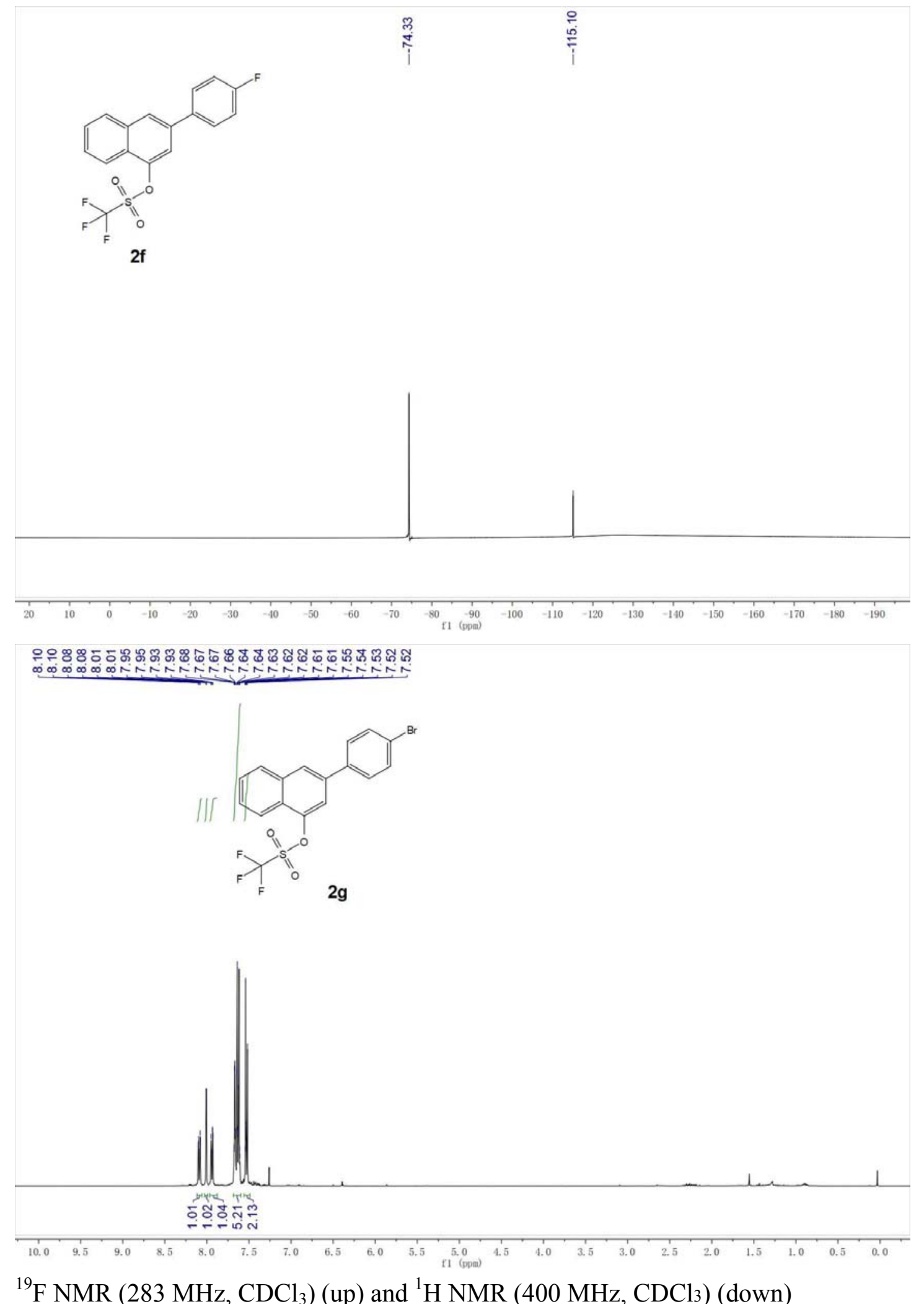

${ }^{19} \mathrm{~F} \mathrm{NMR}\left(283 \mathrm{MHz}, \mathrm{CDCl}_{3}\right.$ ) (up) and ${ }^{1} \mathrm{H} \mathrm{NMR}\left(400 \mathrm{MHz}, \mathrm{CDCl}_{3}\right)$ (down) 

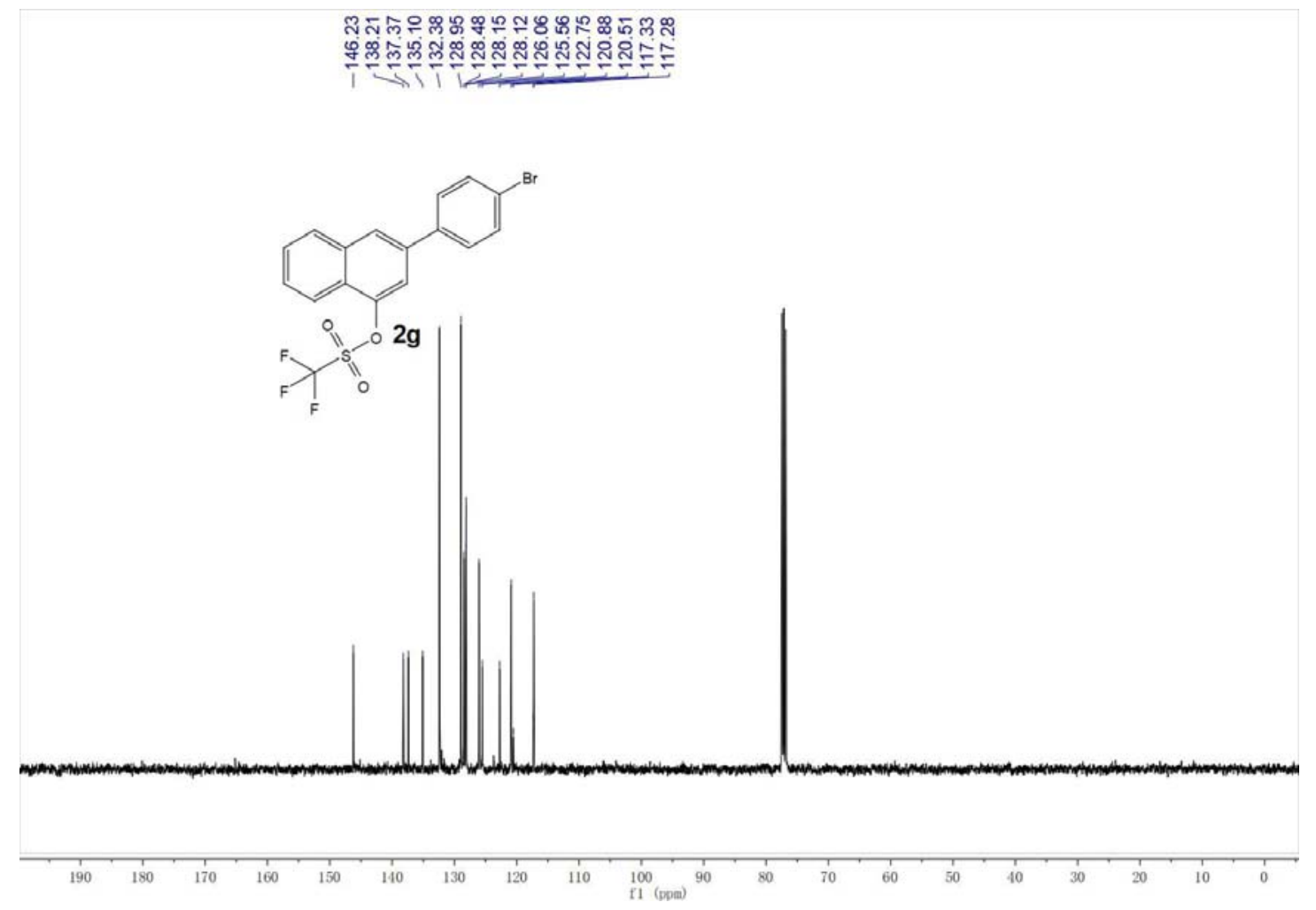

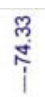
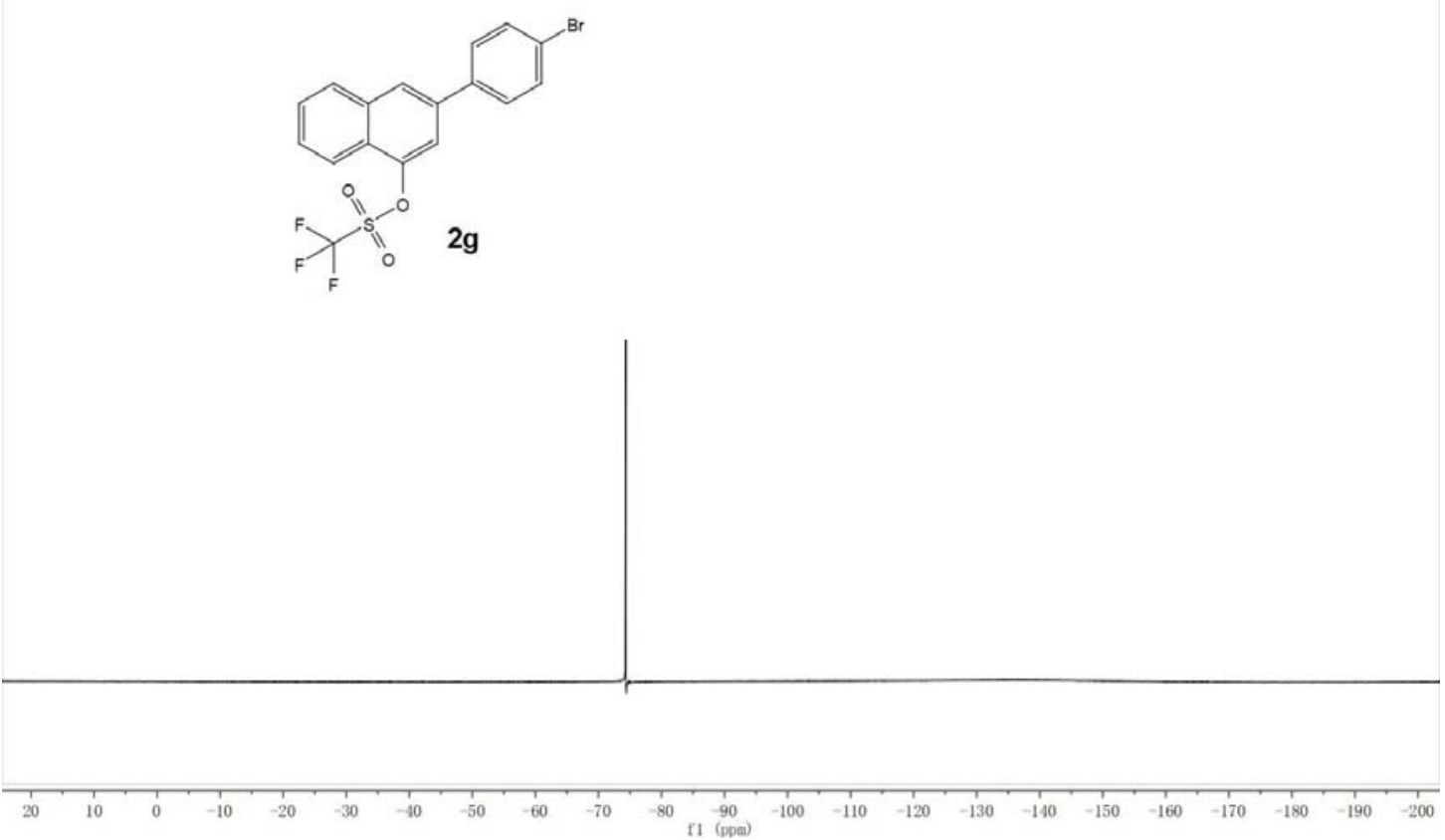

${ }^{13} \mathrm{C}$ NMR (101 MHz, CDCl 3 ) (up) and ${ }^{19} \mathrm{~F}$ NMR (565 MHz, No Solvent) (down) 


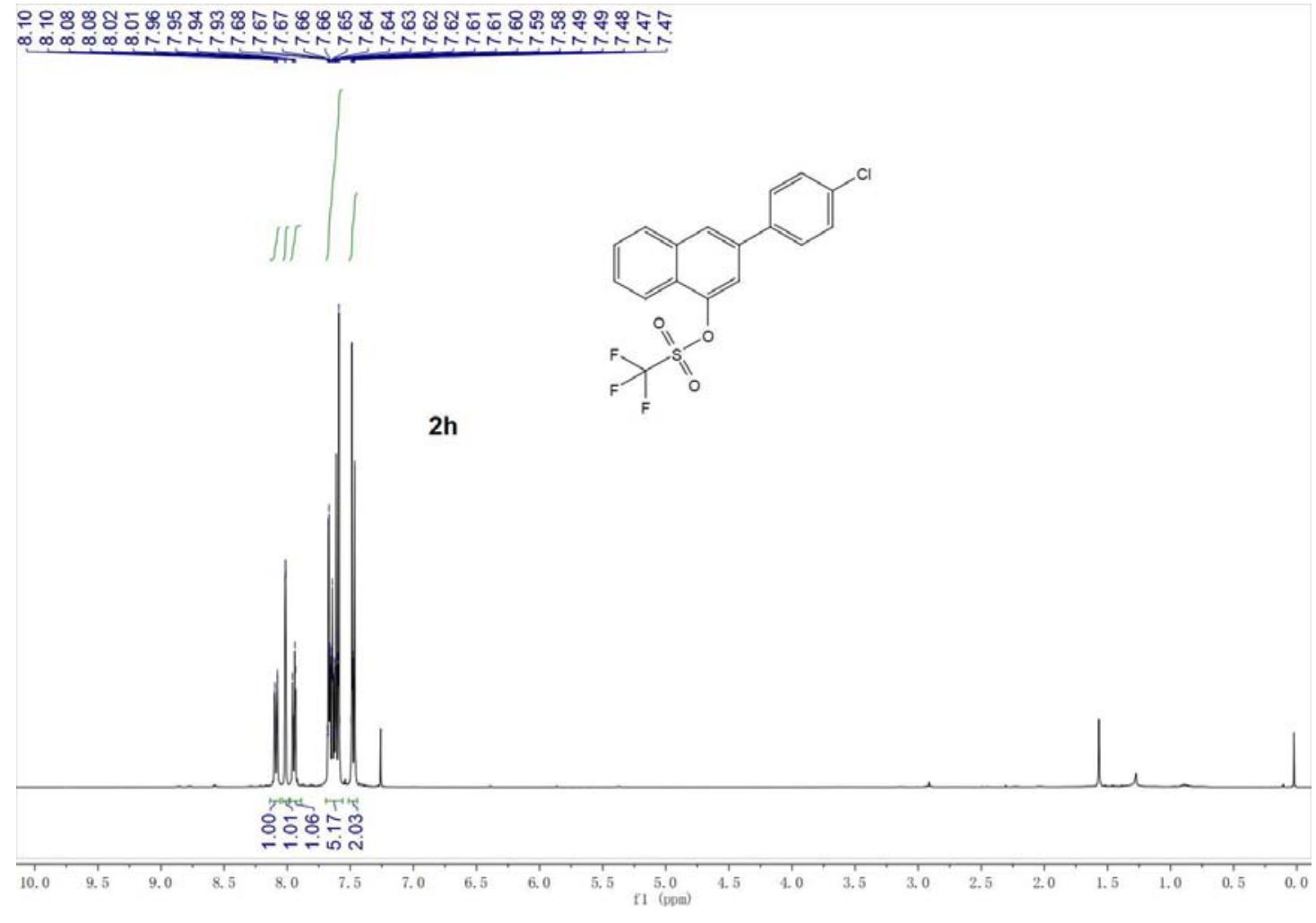

สำ

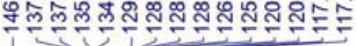
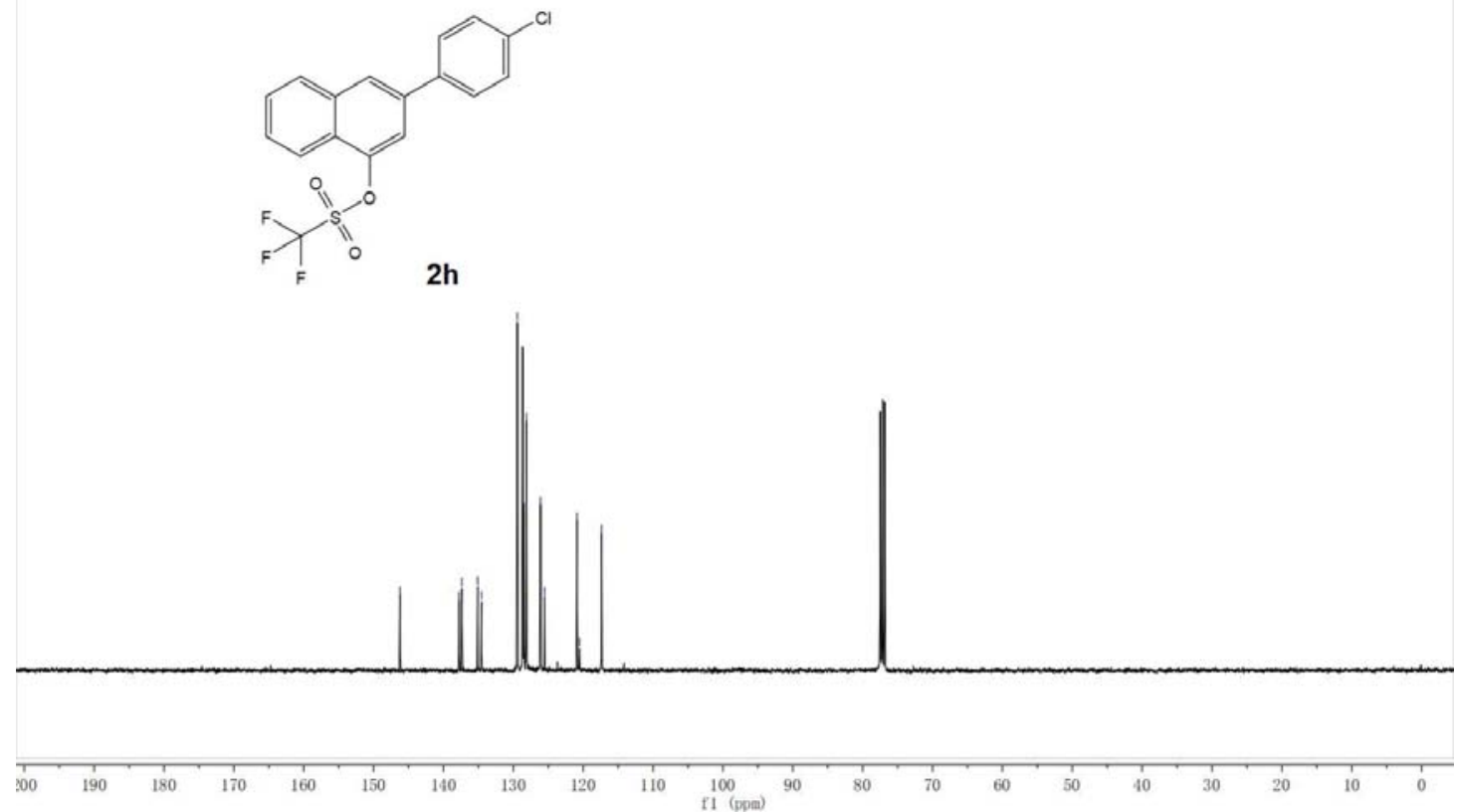

${ }^{1} \mathrm{H}$ NMR (400 MHz, $\mathrm{CDCl}_{3}$ ) (up) and ${ }^{13} \mathrm{C} \mathrm{NMR} \mathrm{(101} \mathrm{MHz,} \mathrm{CDCl}_{3}$ ) (down) 


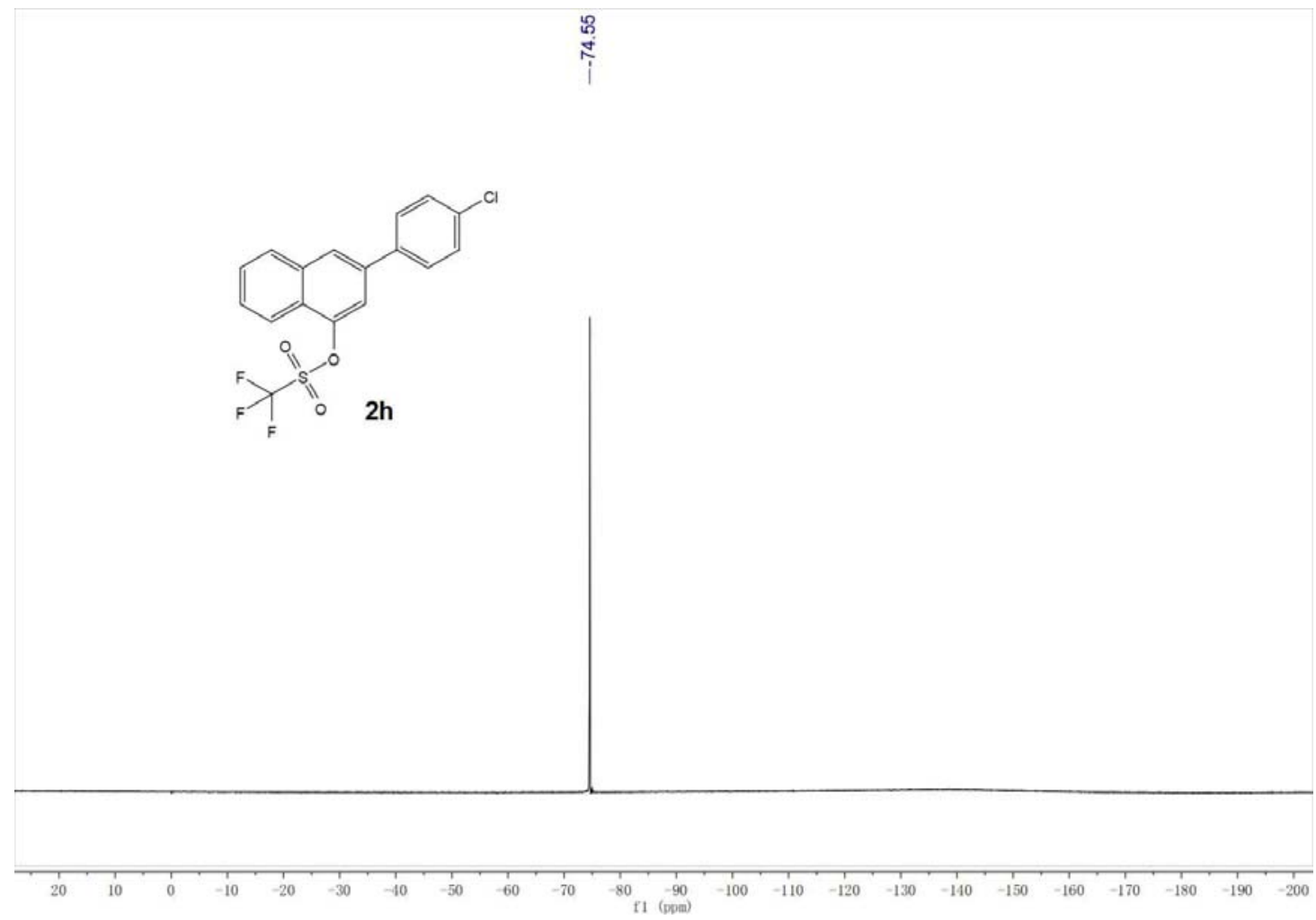

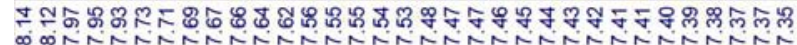

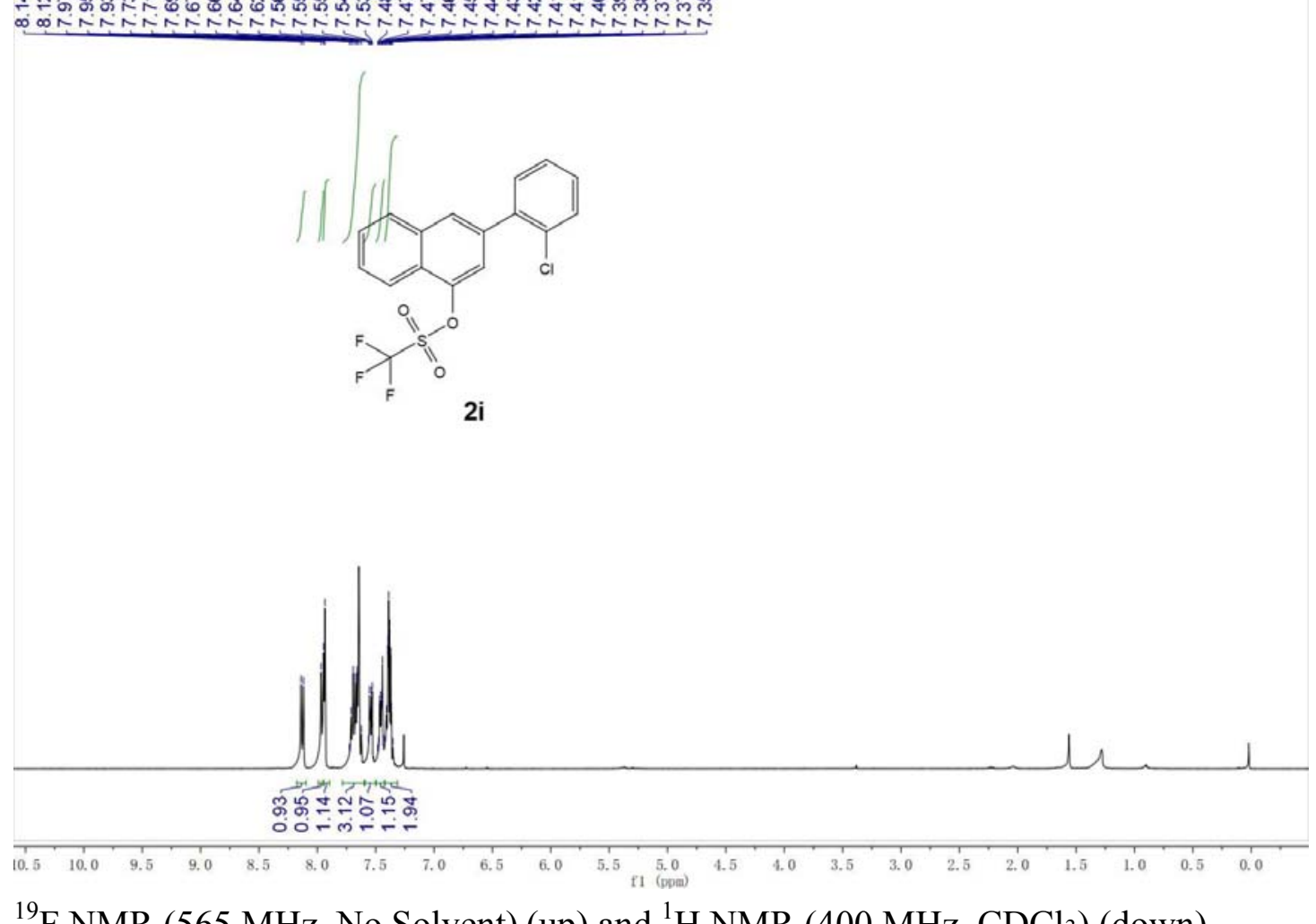

${ }^{19}$ F NMR (565 MHz, No Solvent) (up) and ${ }^{1} \mathrm{H}$ NMR (400 MHz, $\mathrm{CDCl}_{3}$ ) (down) 

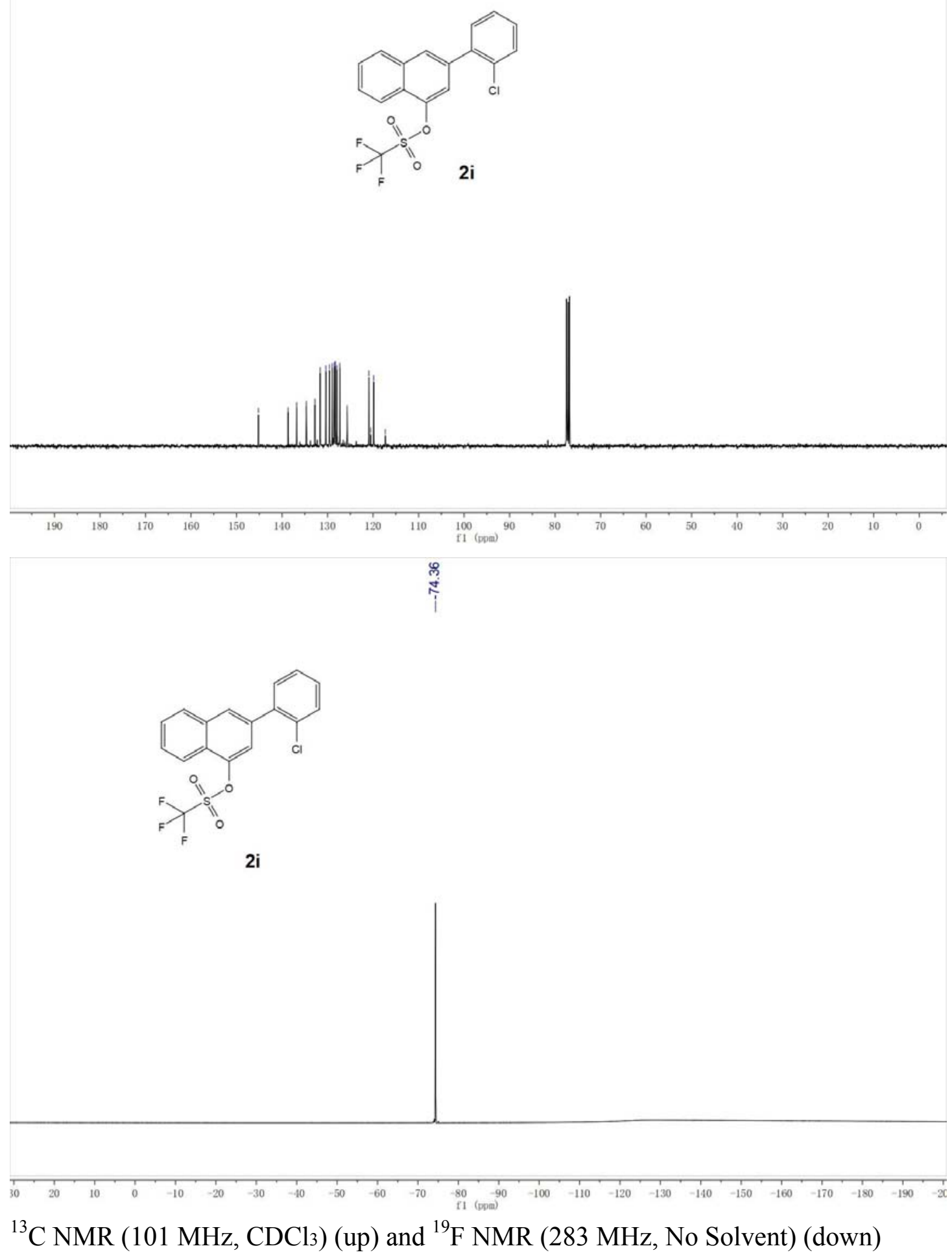


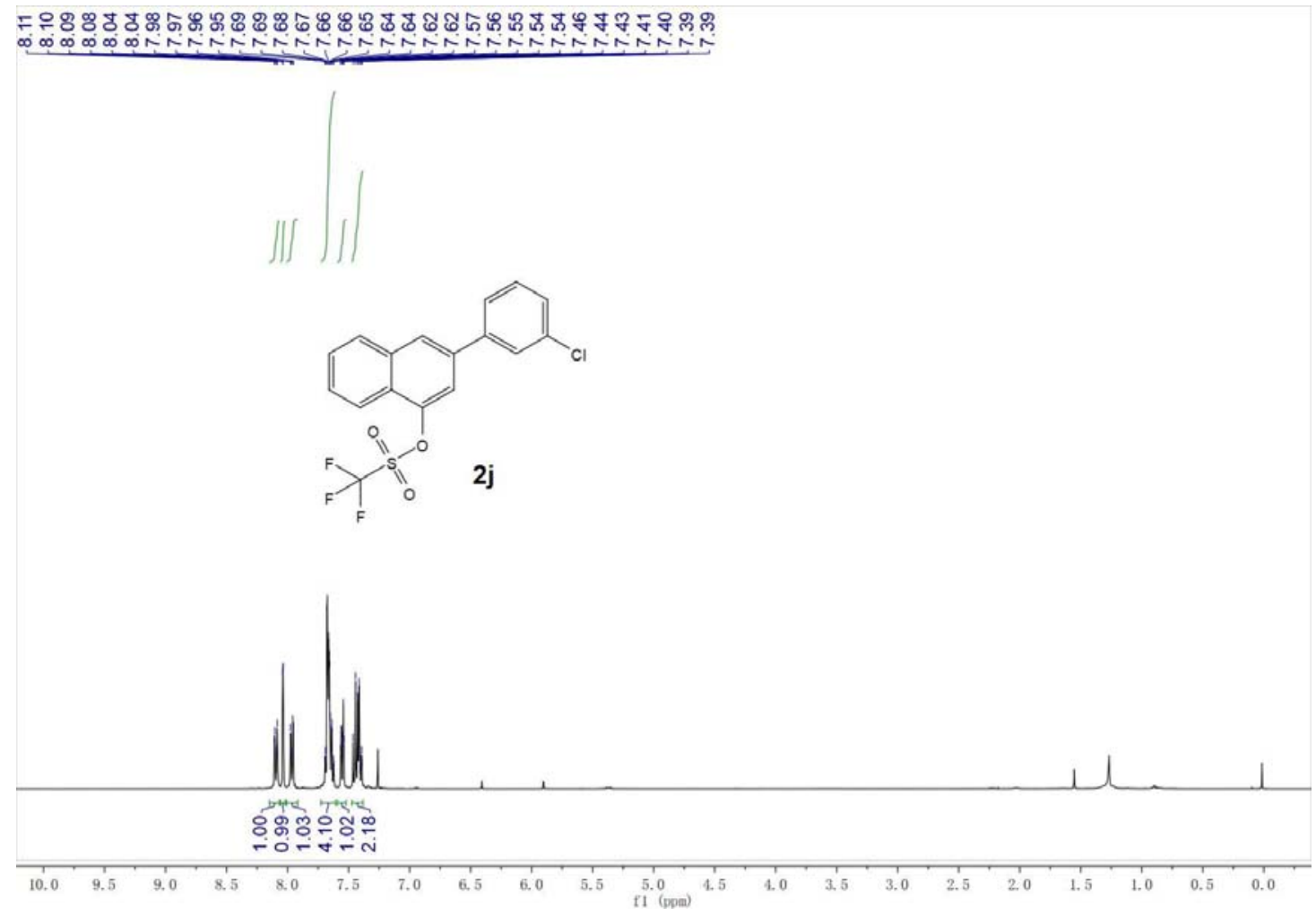

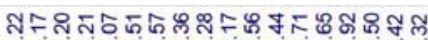

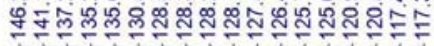
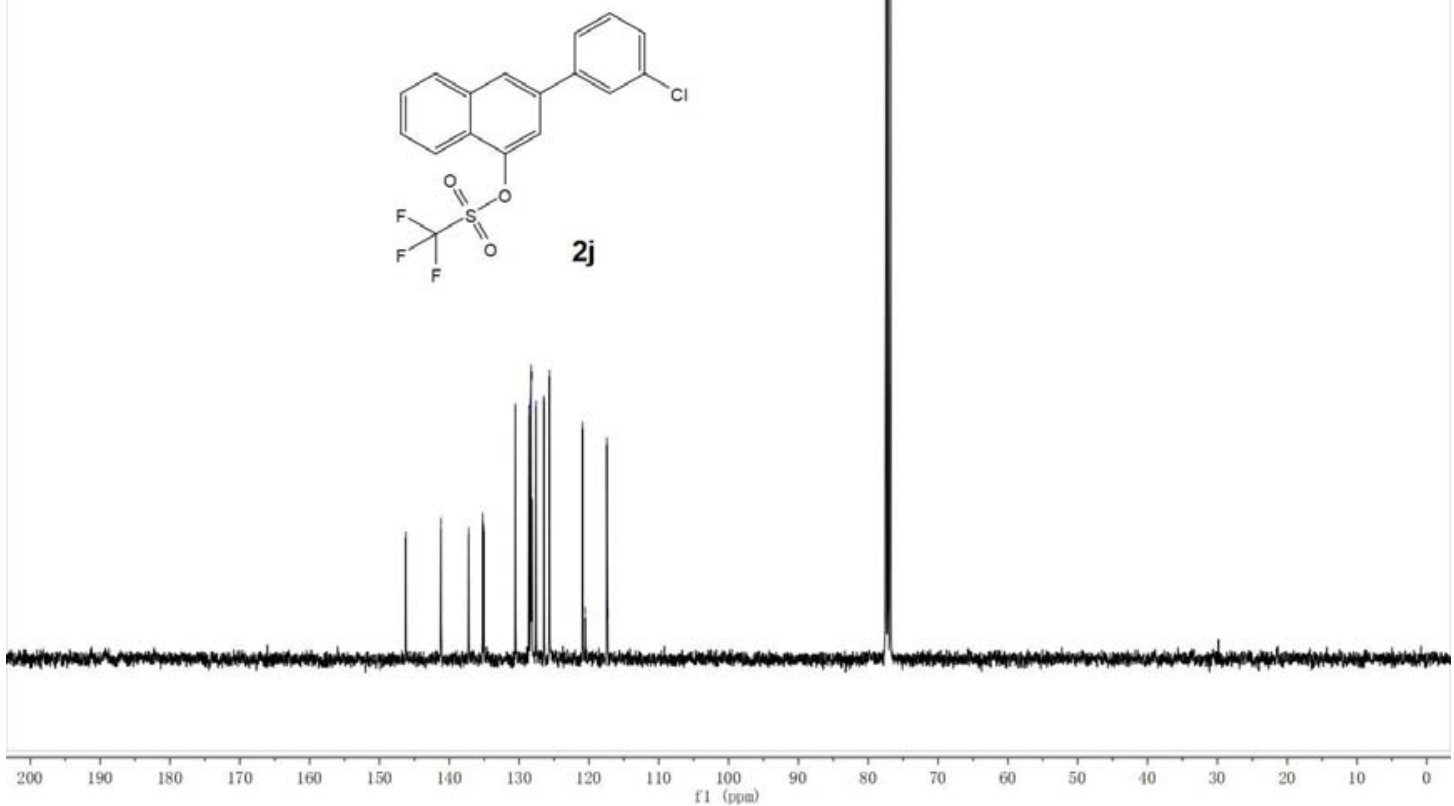

${ }^{1} \mathrm{H} \mathrm{NMR}\left(400 \mathrm{MHz}, \mathrm{CDCl}_{3}\right.$ ) (up) and ${ }^{13} \mathrm{C} \mathrm{NMR}$ (101 MHz, $\mathrm{CDCl}_{3}$ ) (down) 


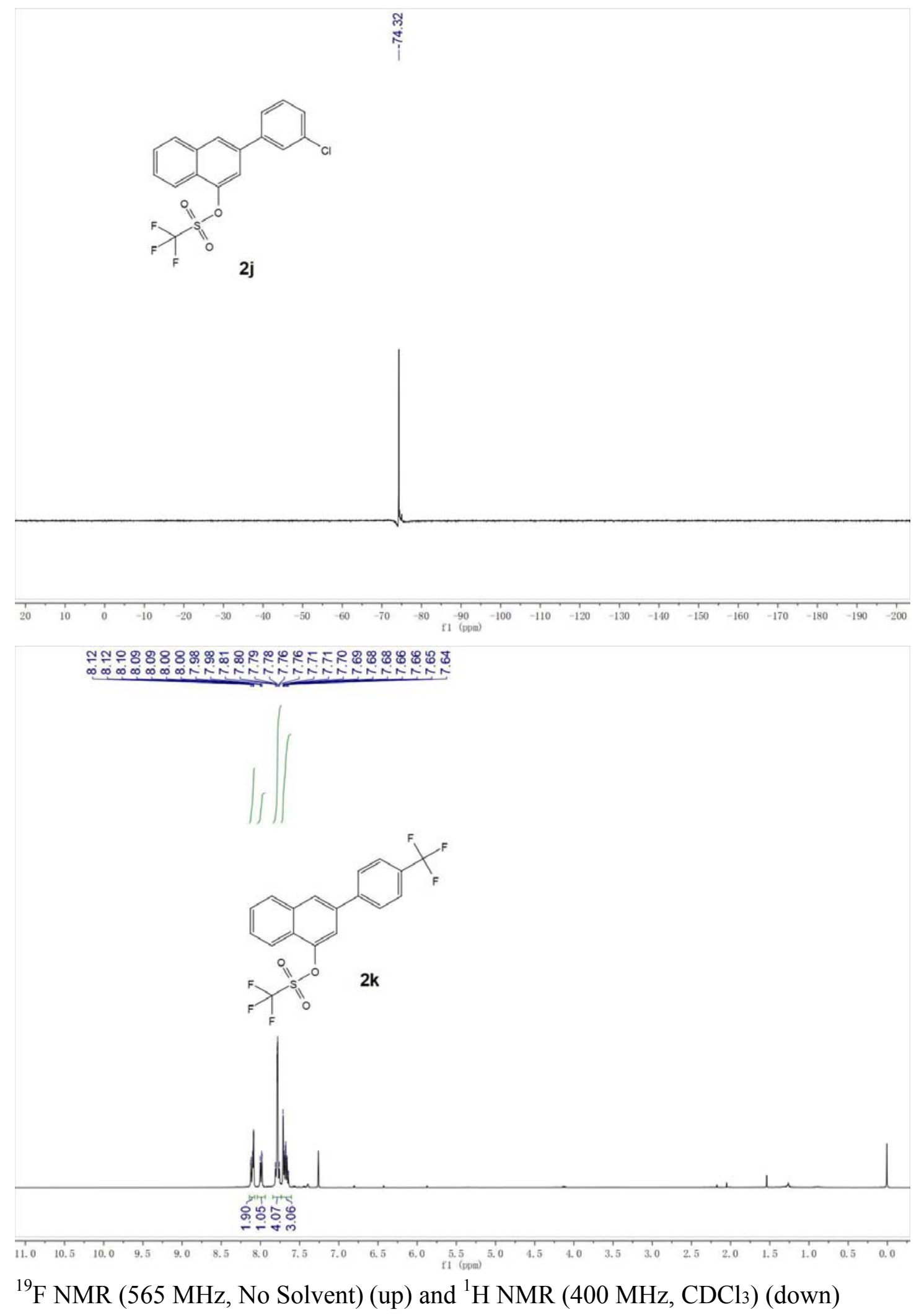



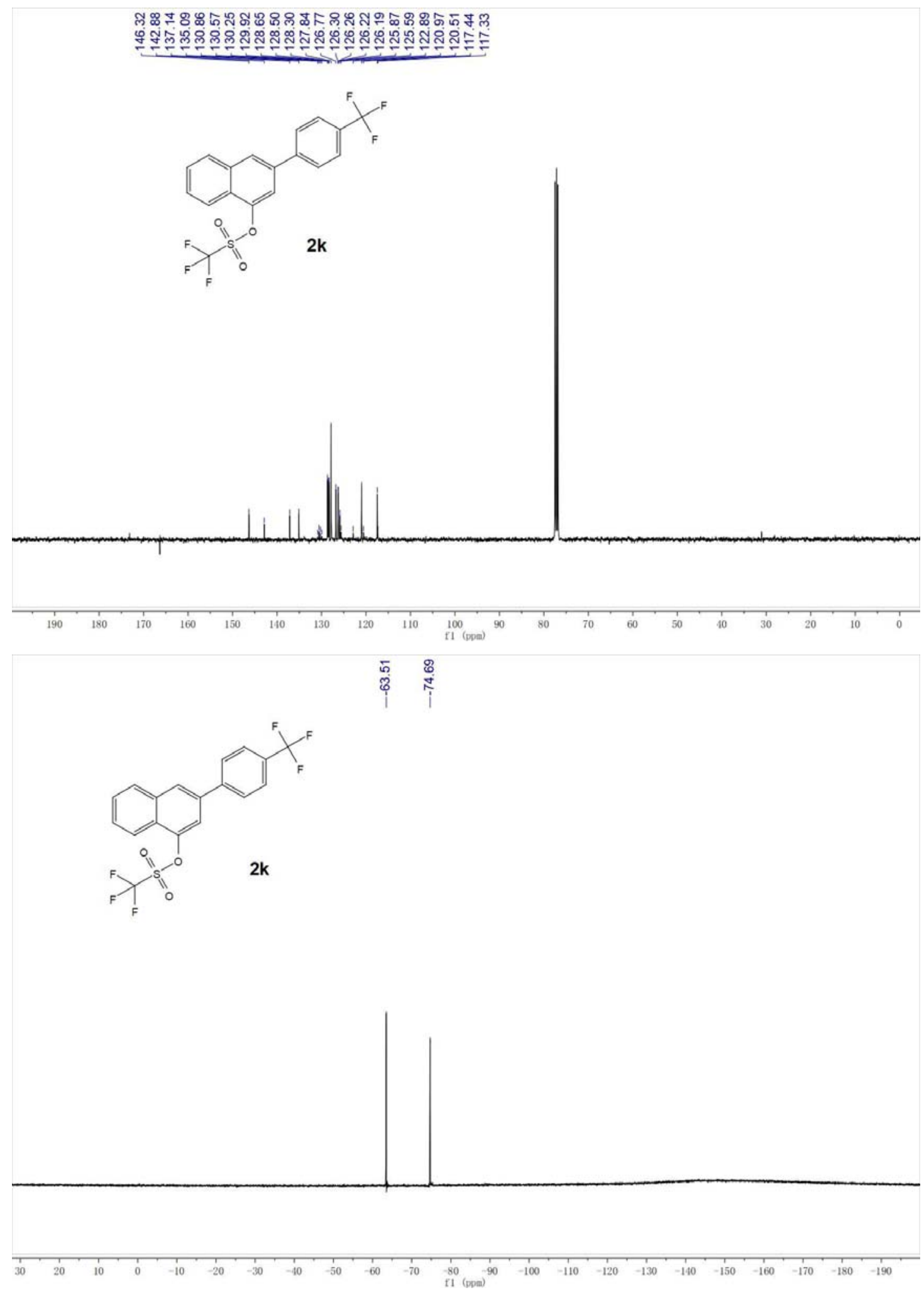

${ }^{13} \mathrm{C}$ NMR (101 MHz, $\mathrm{CDCl}_{3}$ ) (up) and ${ }^{19} \mathrm{~F} \mathrm{NMR}$ (565 MHz, No Solvent) (down) 


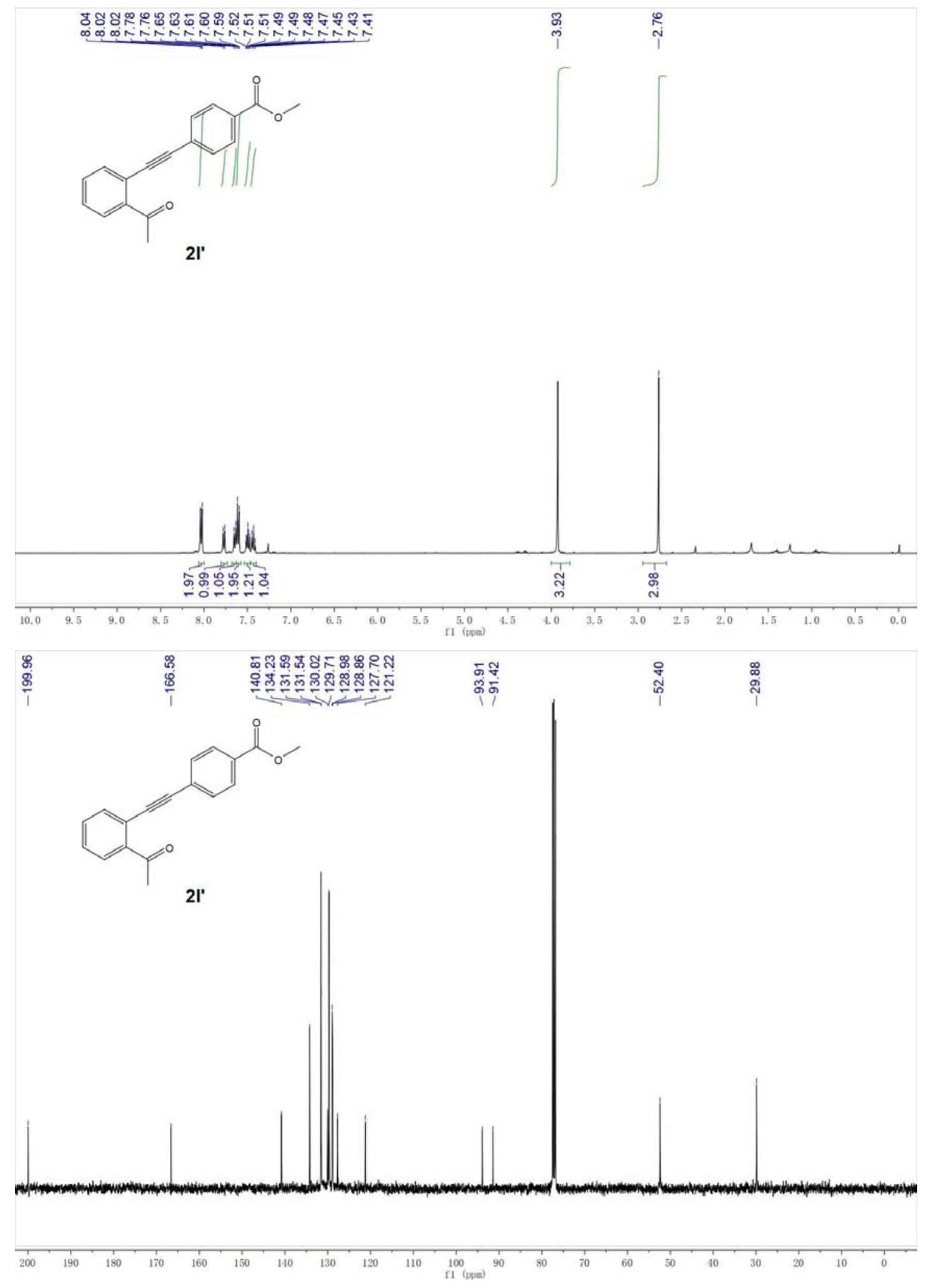

${ }^{1} \mathrm{H}$ NMR (400 MHz, $\mathrm{CDCl}_{3}$ ) (up) and ${ }^{13} \mathrm{C} \mathrm{NMR} \mathrm{(101} \mathrm{MHz,} \mathrm{CDCl}_{3}$ ) (down) 


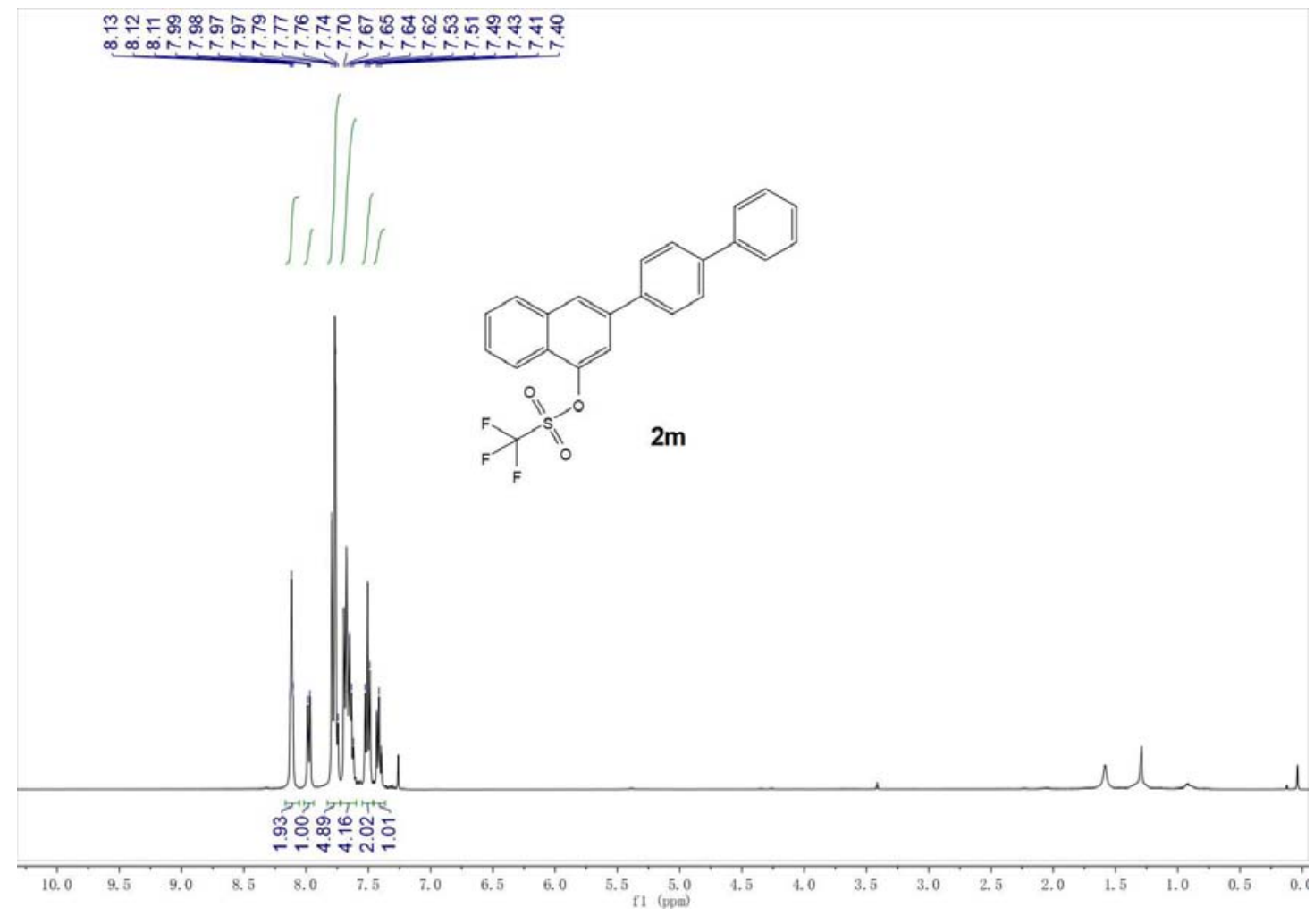

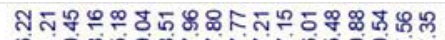

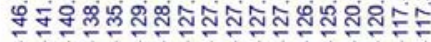
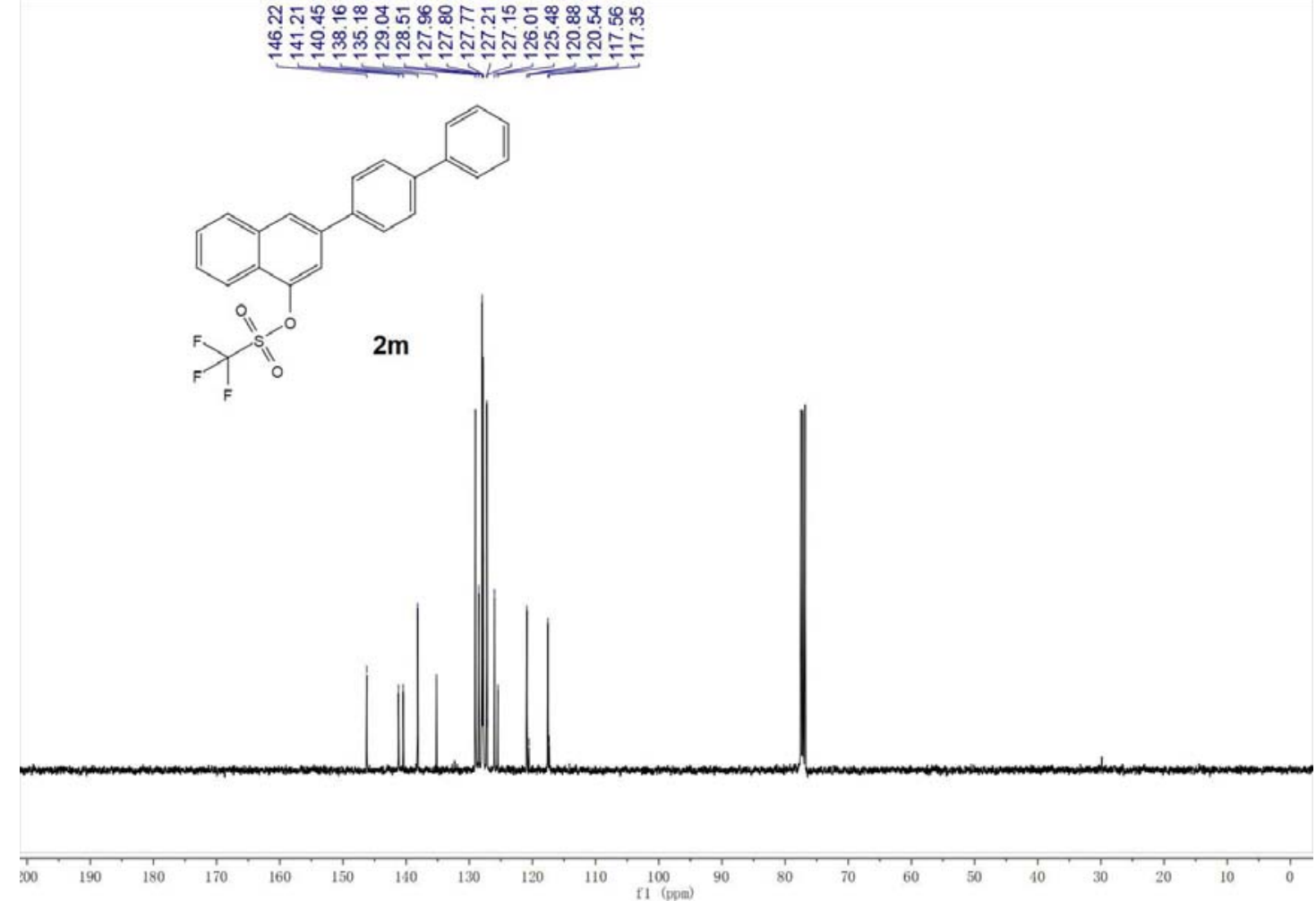

${ }^{1} \mathrm{H}$ NMR (400 MHz, $\mathrm{CDCl}_{3}$ ) (up) and ${ }^{13} \mathrm{C} \mathrm{NMR} \mathrm{(101} \mathrm{MHz,} \mathrm{CDCl}_{3}$ ) (down) 


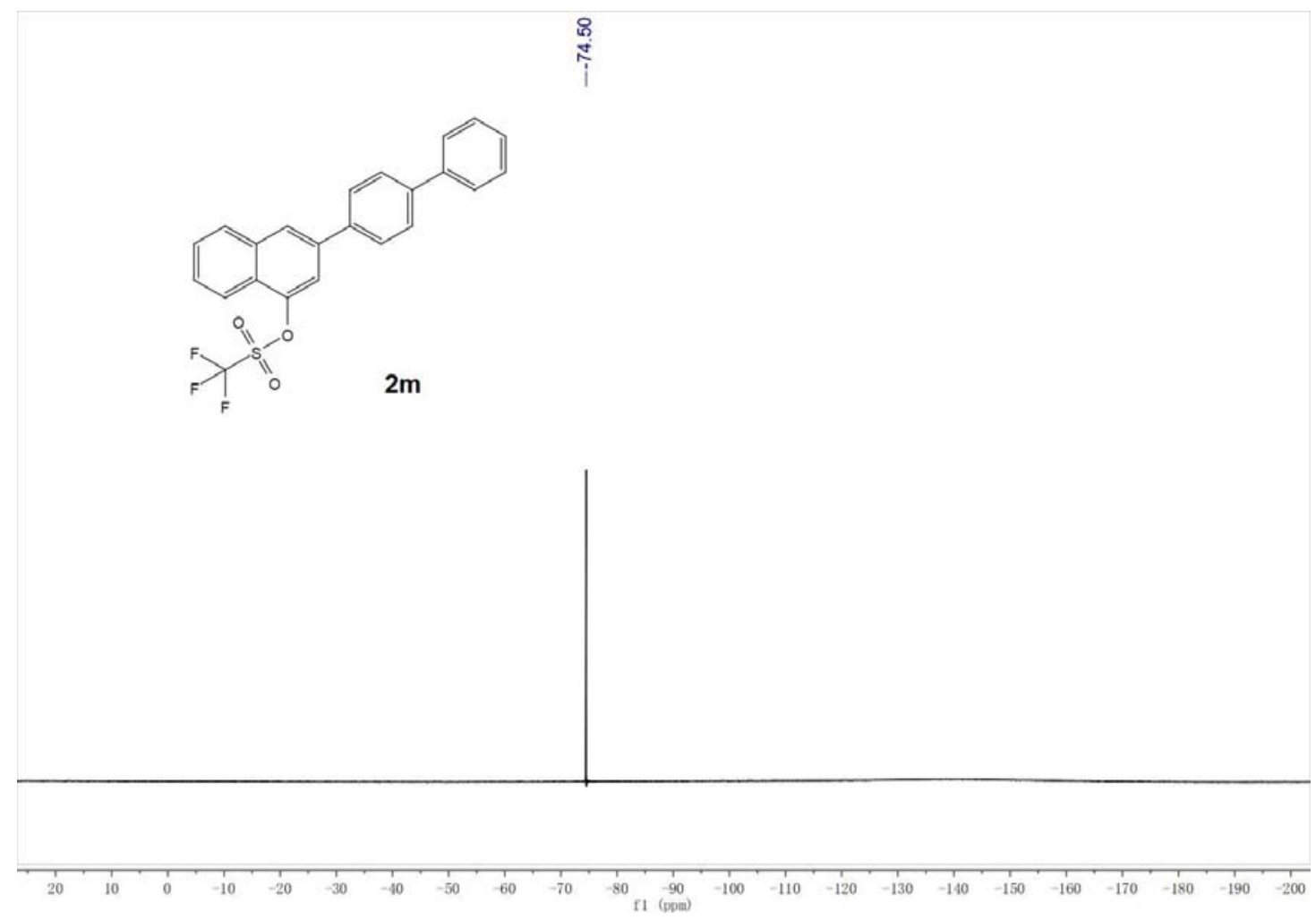

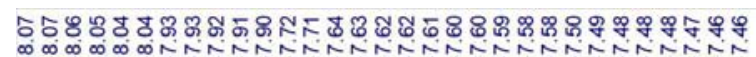

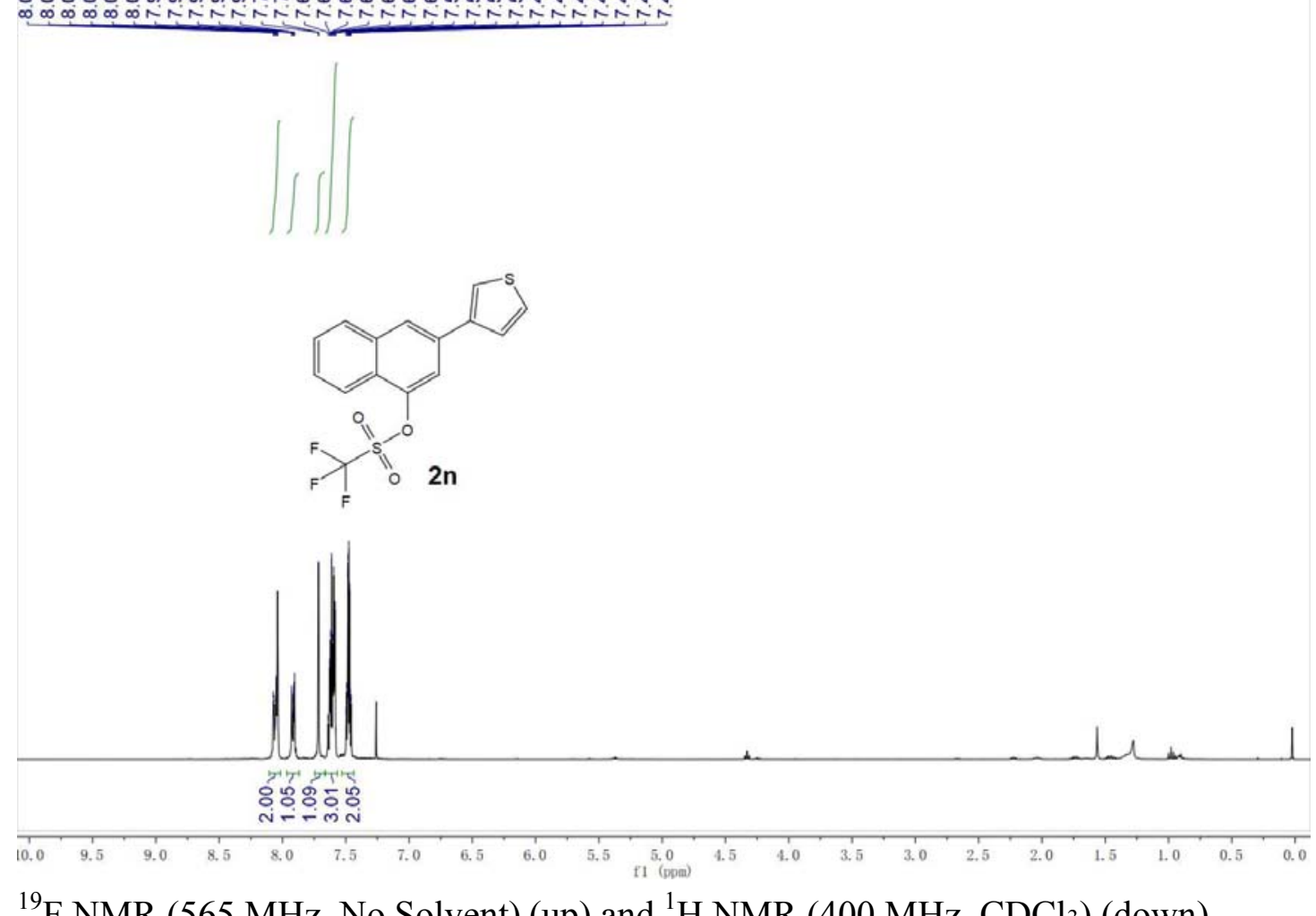

${ }^{19}$ F NMR (565 MHz, No Solvent) (up) and ${ }^{1} \mathrm{H}$ NMR (400 MHz, $\mathrm{CDCl}_{3}$ ) (down) 

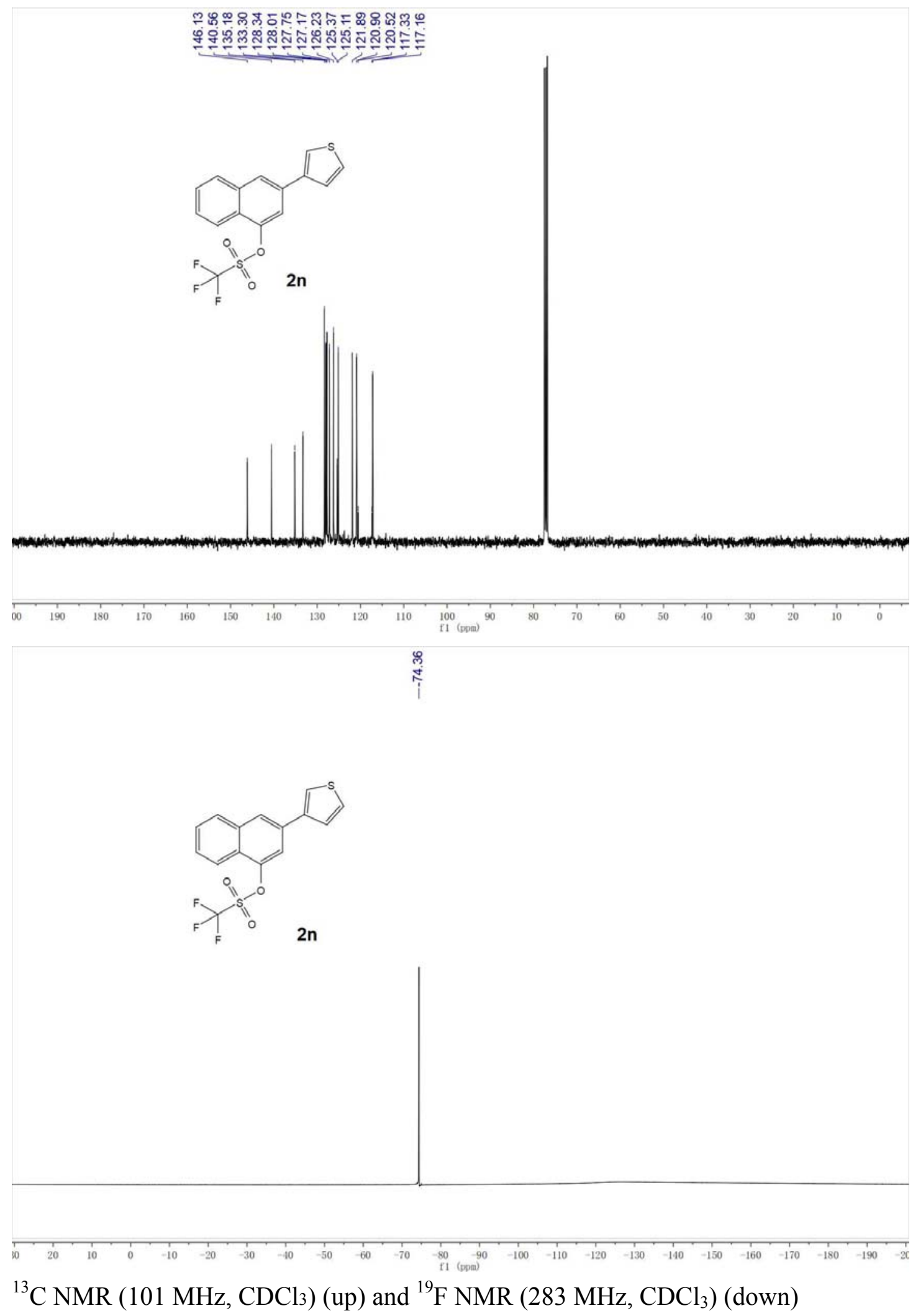

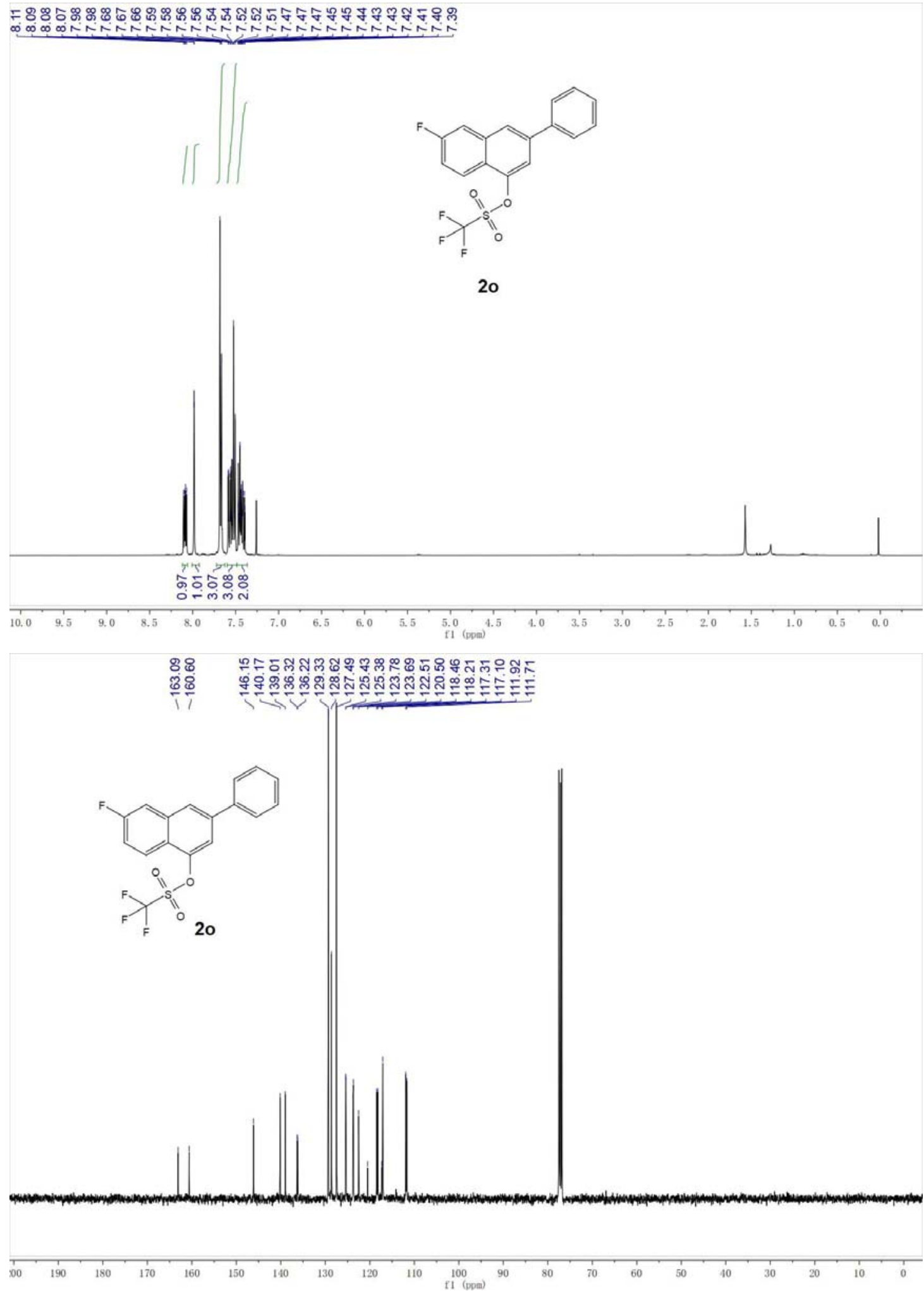

${ }^{1} \mathrm{H} \mathrm{NMR}\left(400 \mathrm{MHz}, \mathrm{CDCl}_{3}\right.$ ) (up) and ${ }^{13} \mathrm{C} \mathrm{NMR}$ (101 MHz, $\mathrm{CDCl}_{3}$ ) (down) 


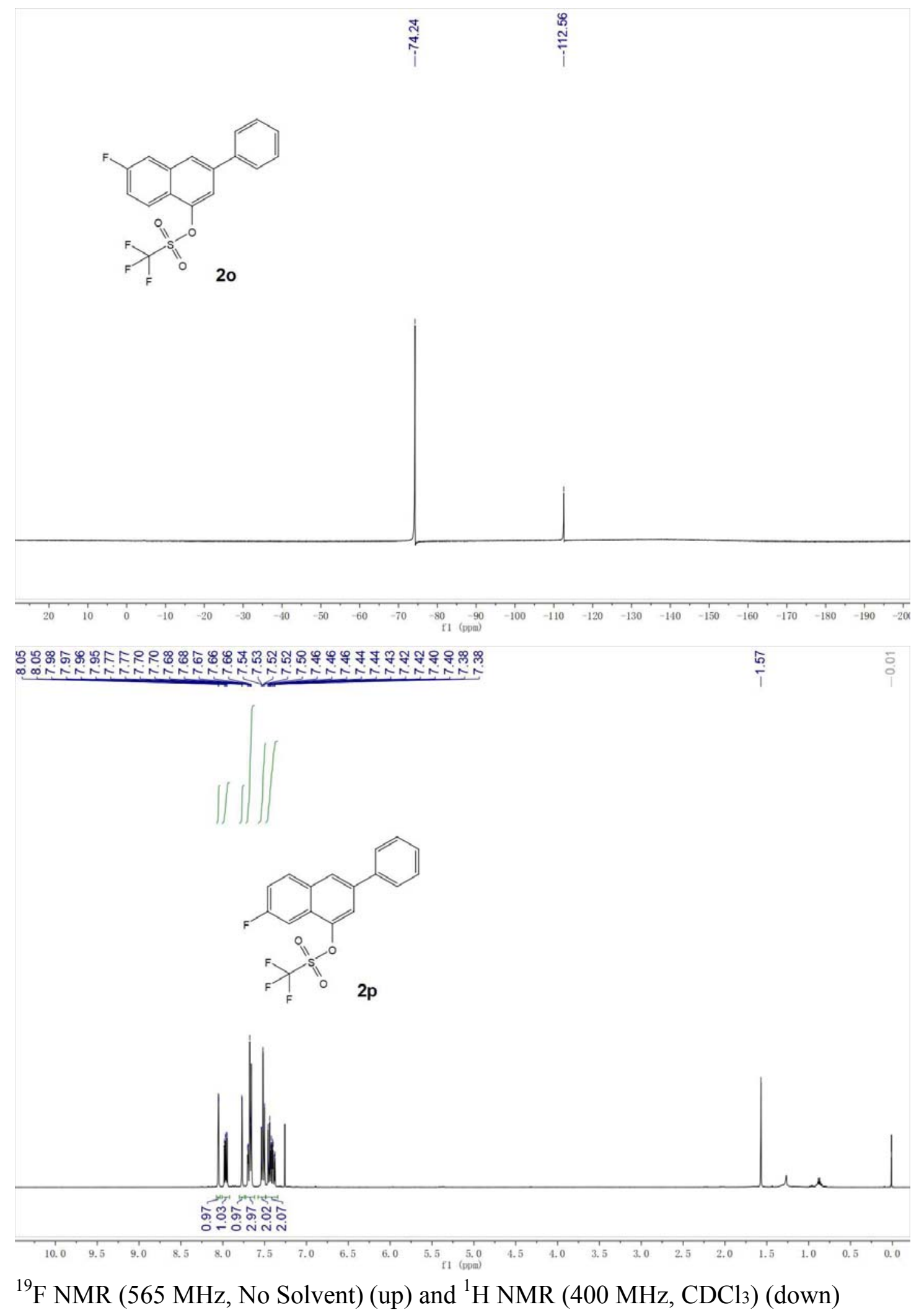



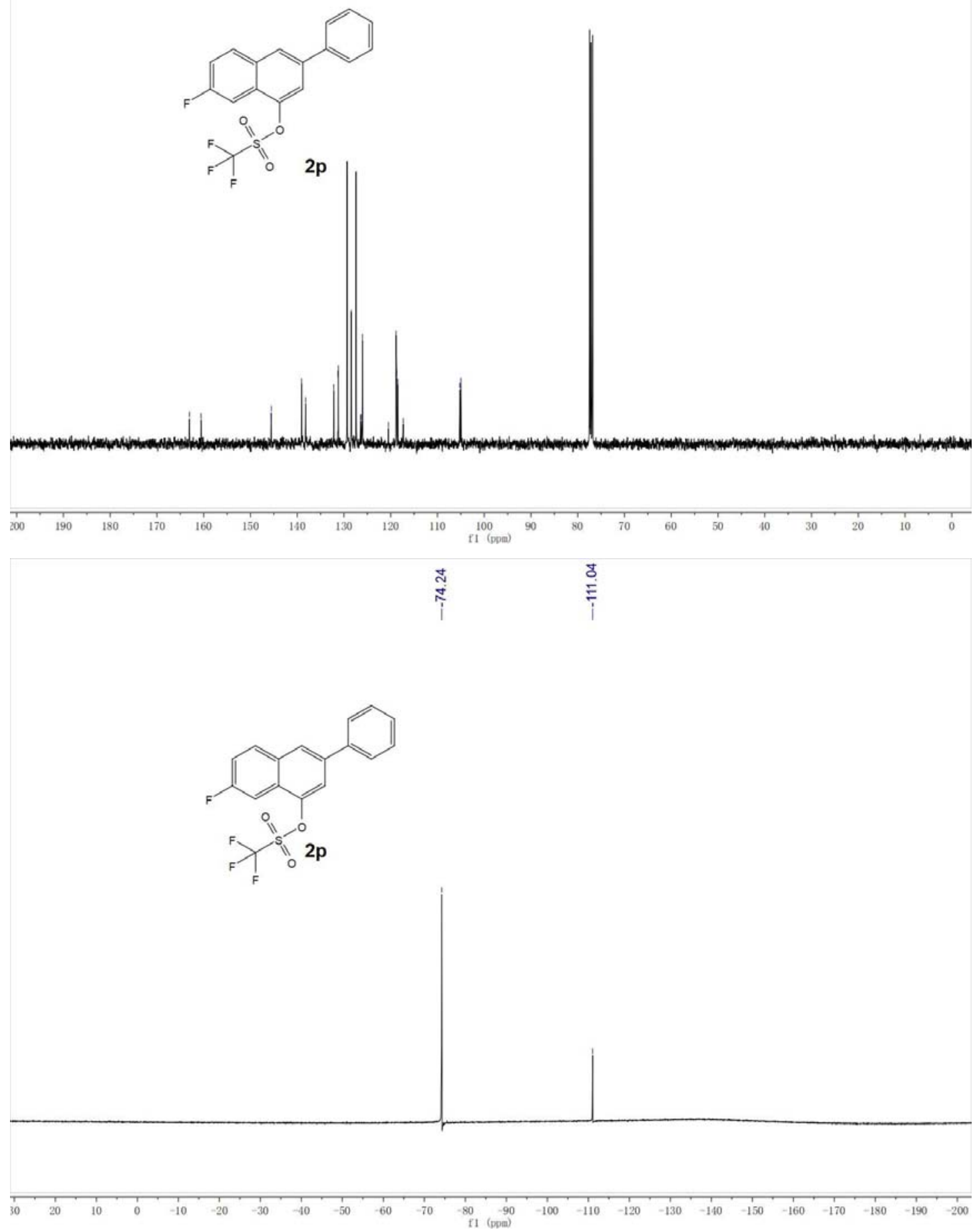

${ }^{13} \mathrm{C}$ NMR (101 MHz, CDCl 3 ) (up) and ${ }^{19} \mathrm{~F}$ NMR (565 MHz, No Solvent) (down) 

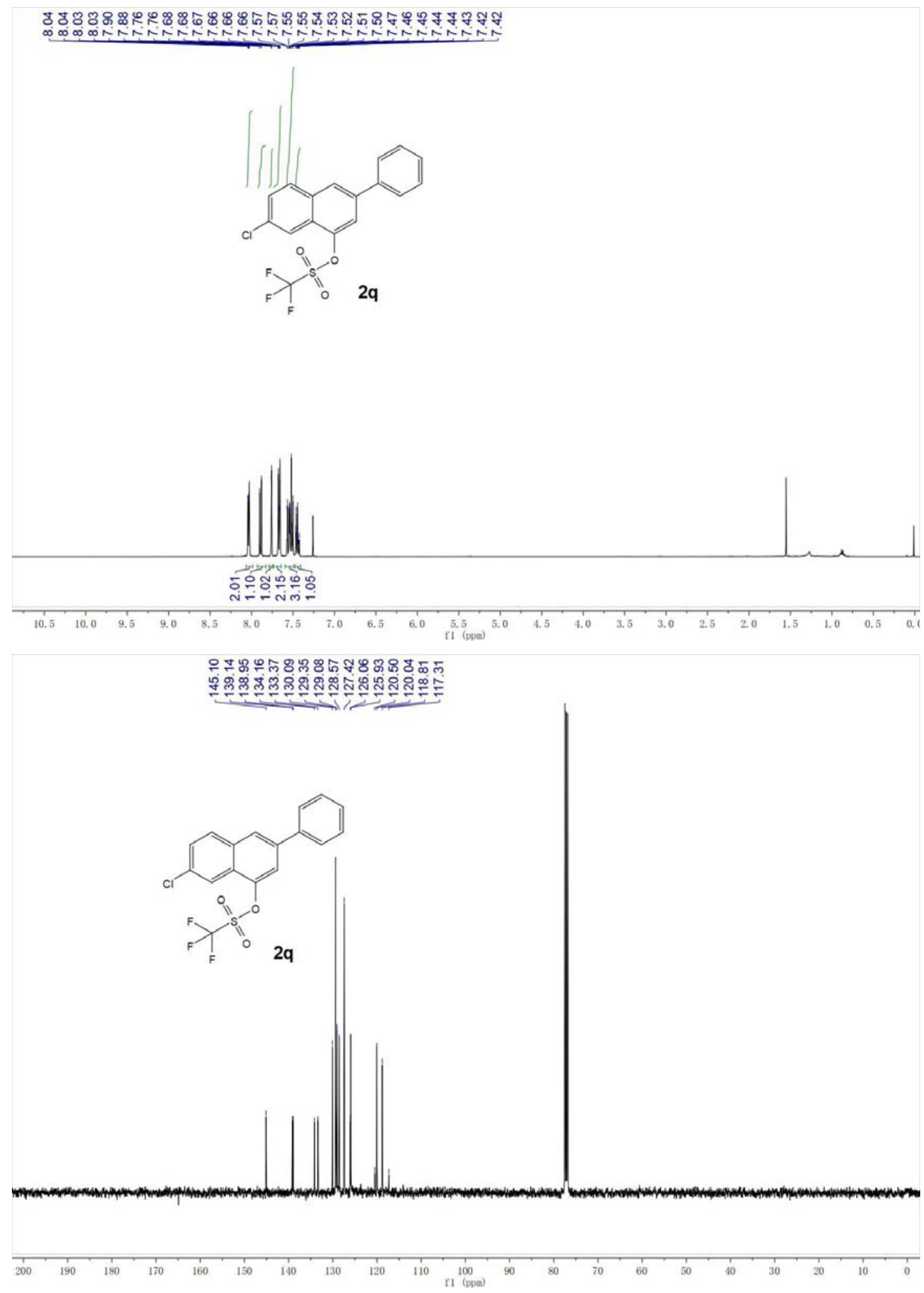

${ }^{1} \mathrm{H}$ NMR (400 MHz, $\mathrm{CDCl}_{3}$ ) (up) and ${ }^{13} \mathrm{C} \mathrm{NMR} \mathrm{(101} \mathrm{MHz,} \mathrm{CDCl}_{3}$ ) (down) 

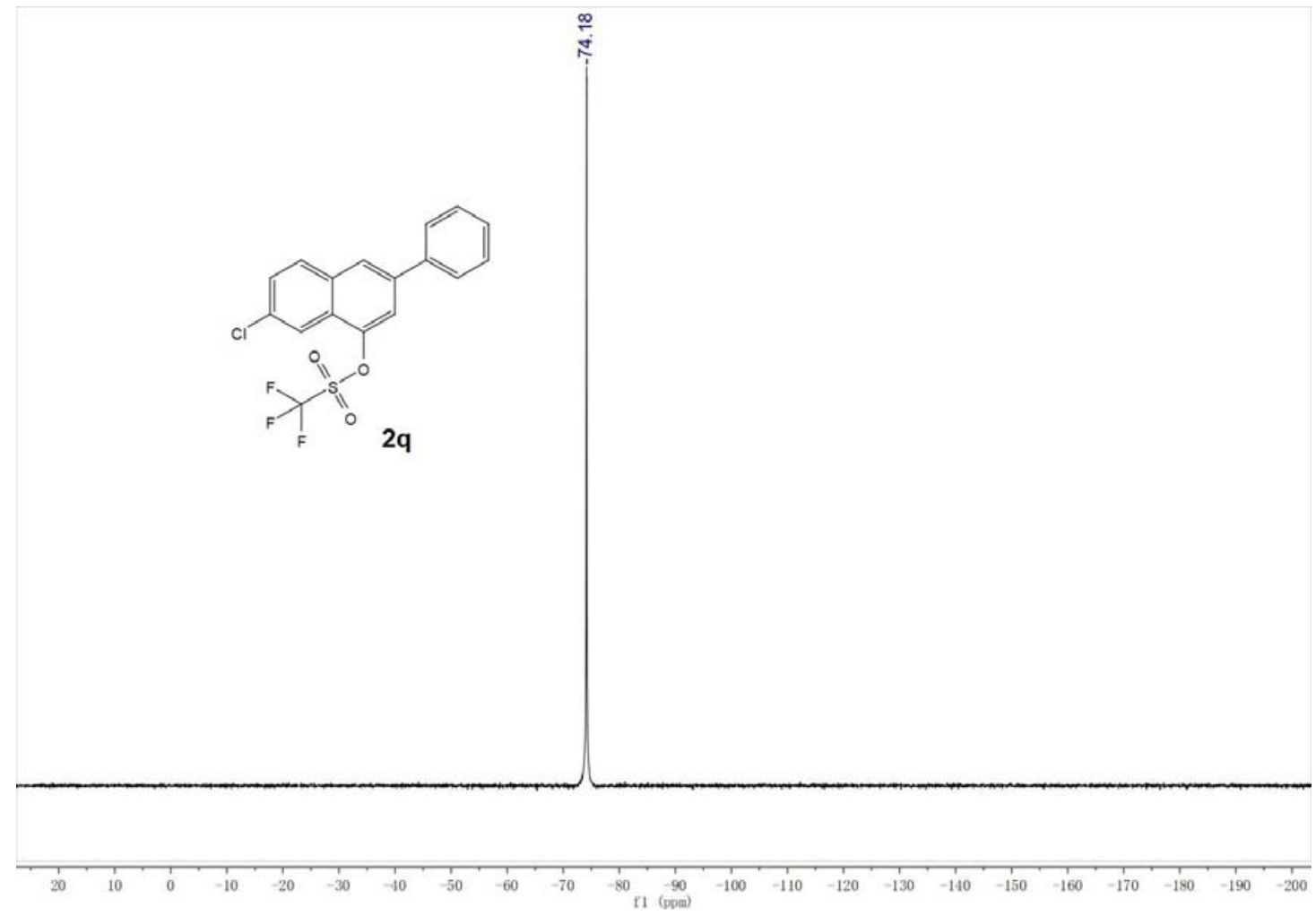

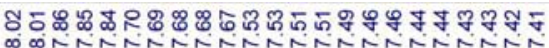
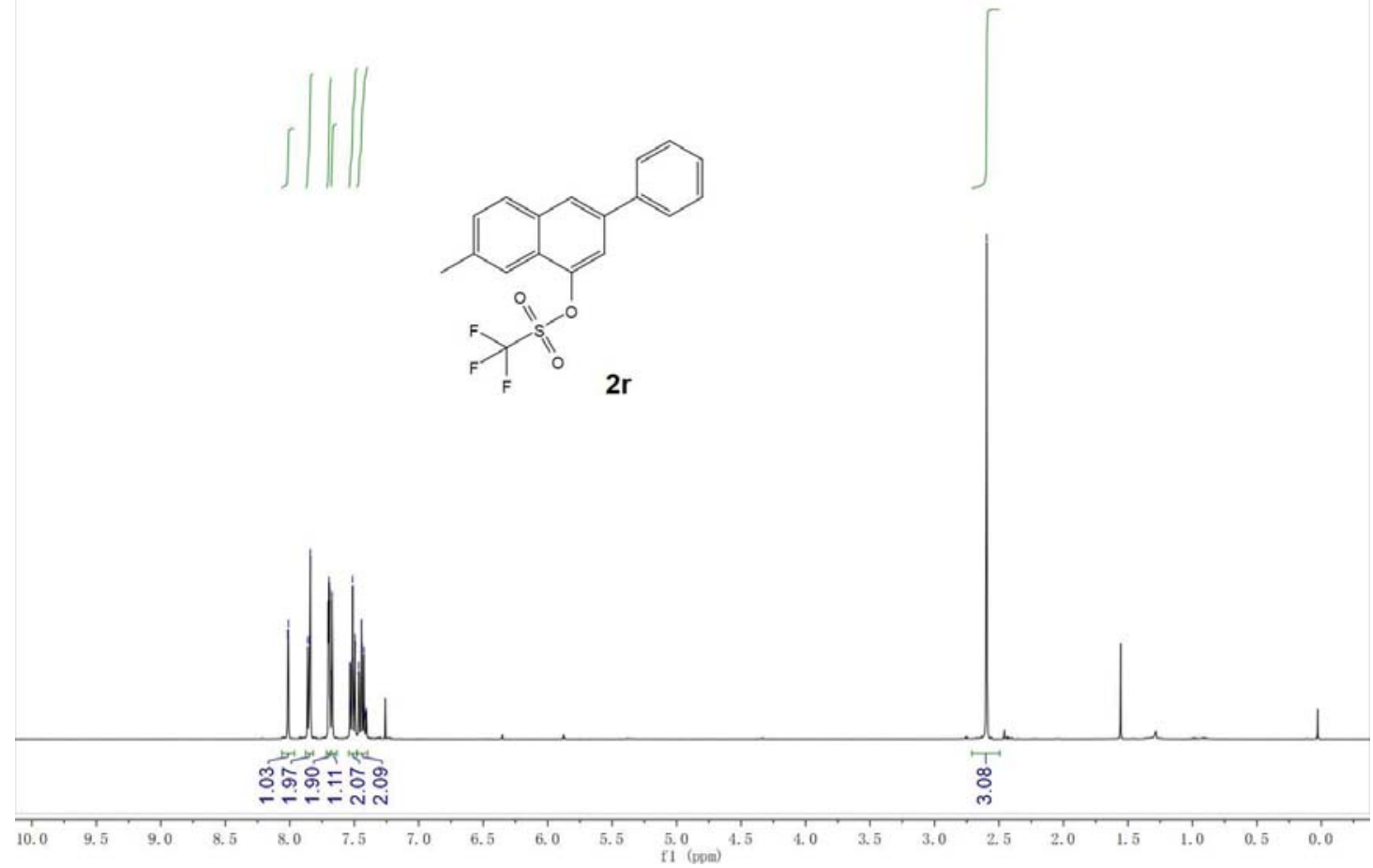

${ }^{19}$ F NMR (565 MHz, No Solvent) (up) and ${ }^{1} \mathrm{H}$ NMR (400 MHz, CDCl 3 ) (down) 

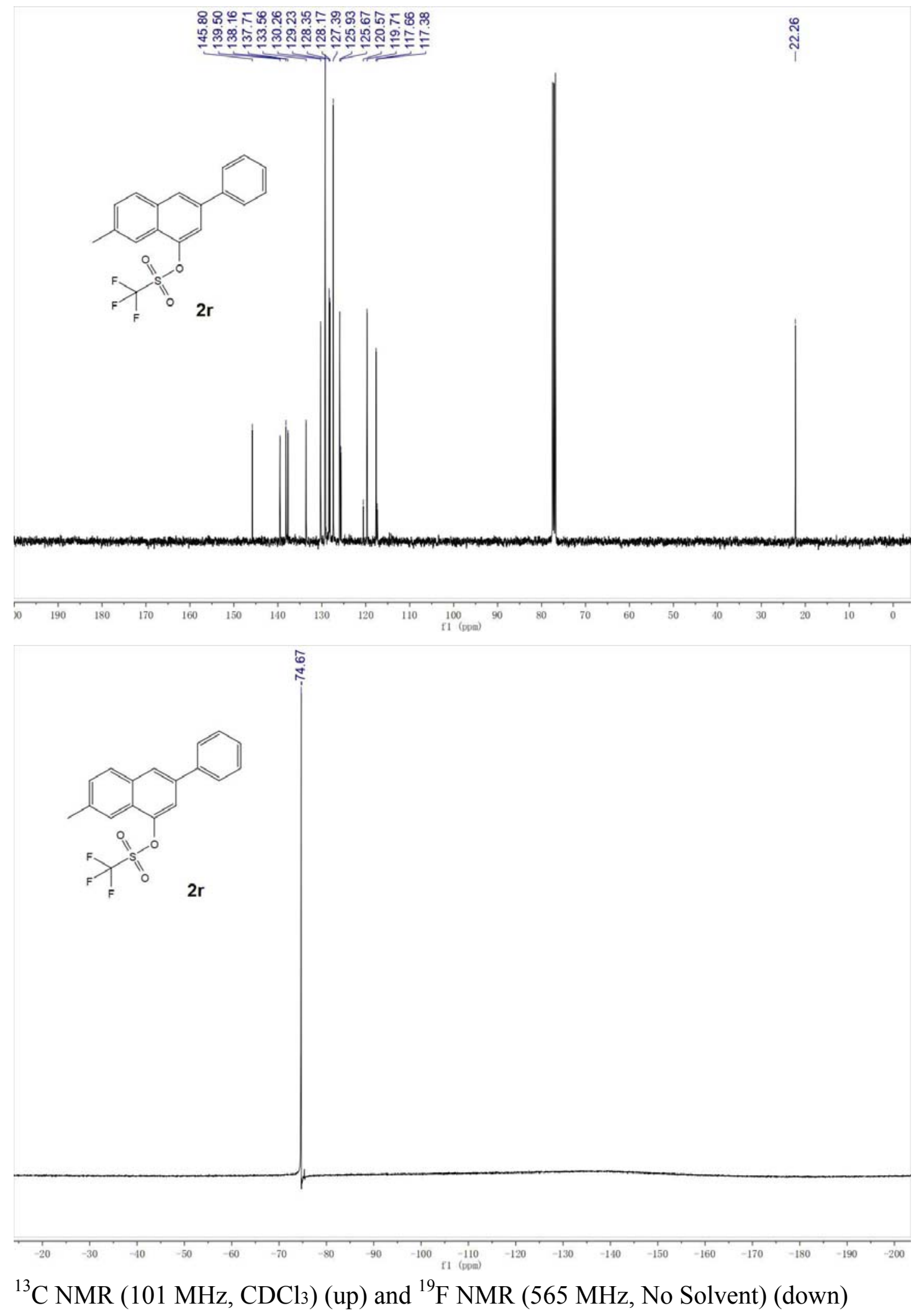

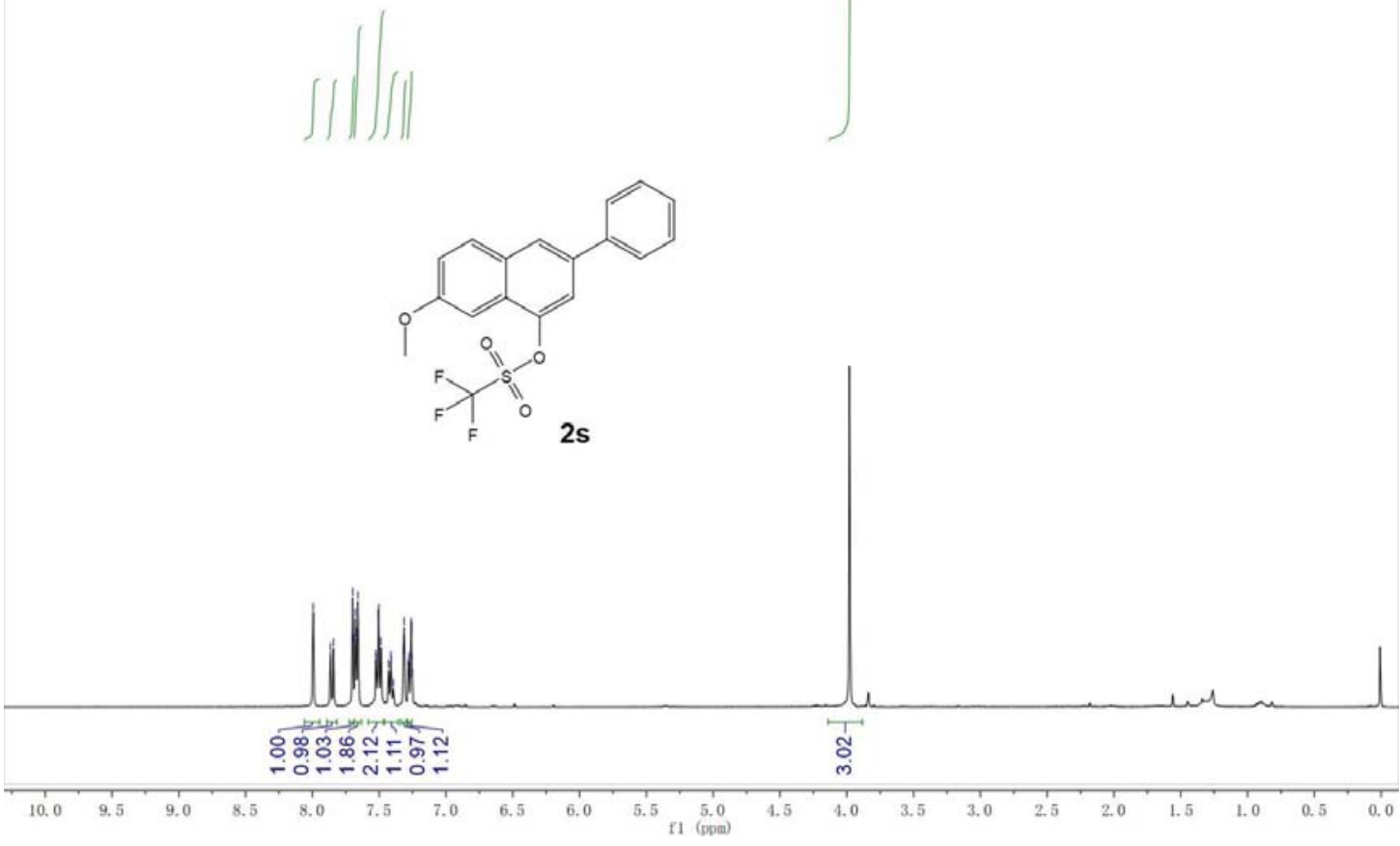

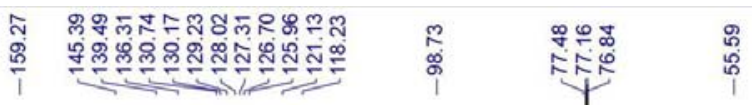

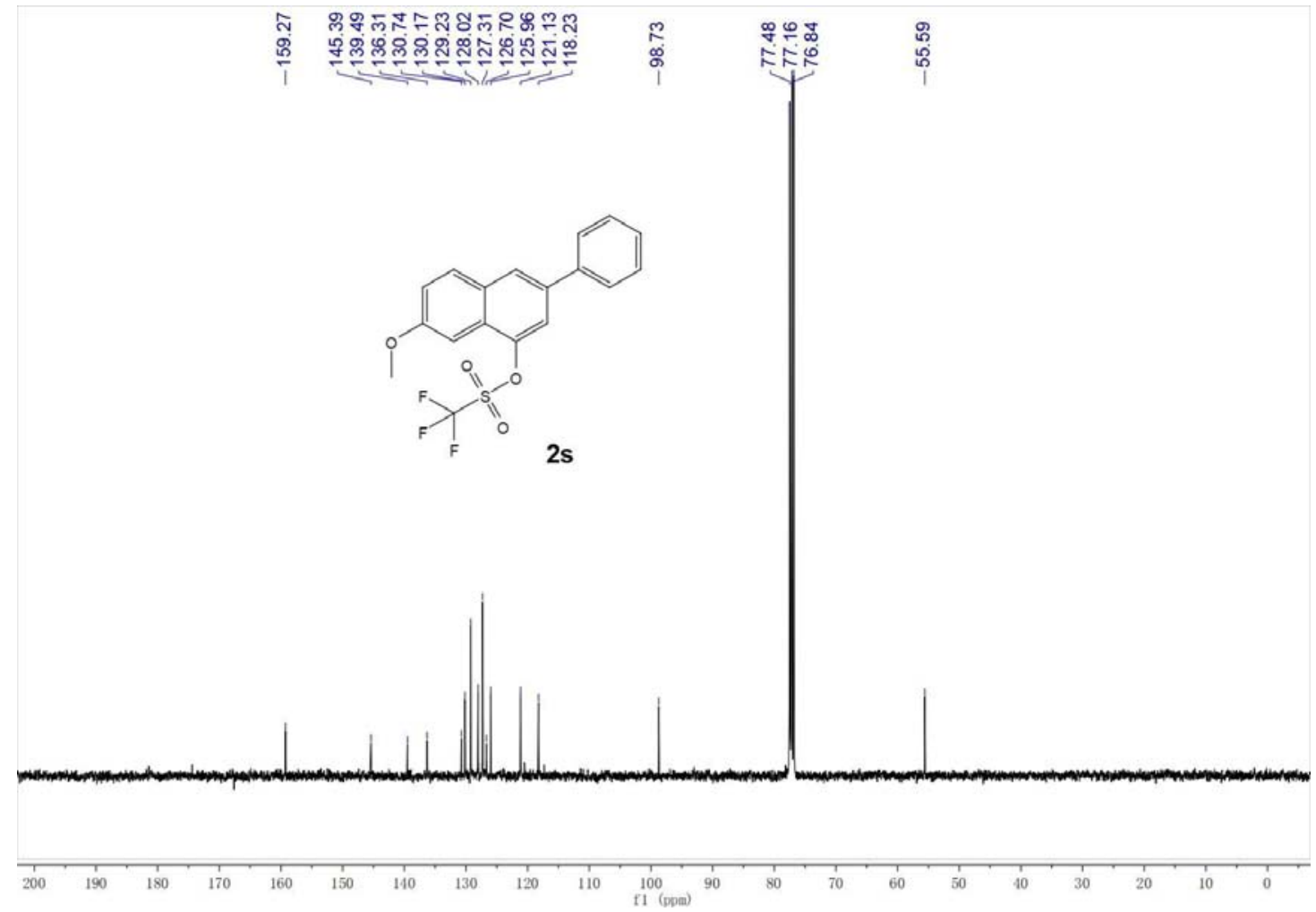

${ }^{1} \mathrm{H} \mathrm{NMR}\left(400 \mathrm{MHz}, \mathrm{CDCl}_{3}\right.$ ) (up) and ${ }^{13} \mathrm{C} \mathrm{NMR}$ (101 MHz, $\mathrm{CDCl}_{3}$ ) (down) 

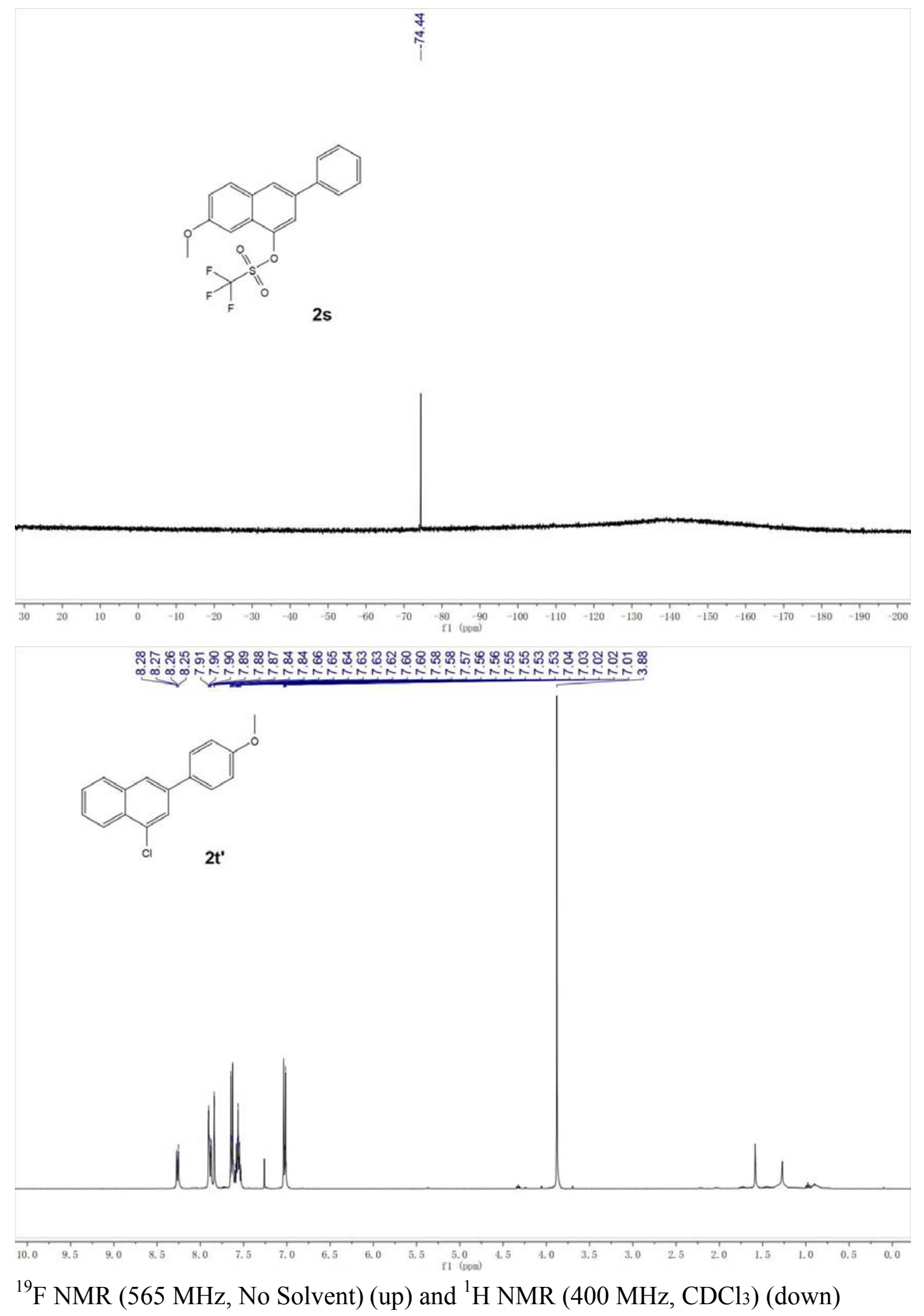


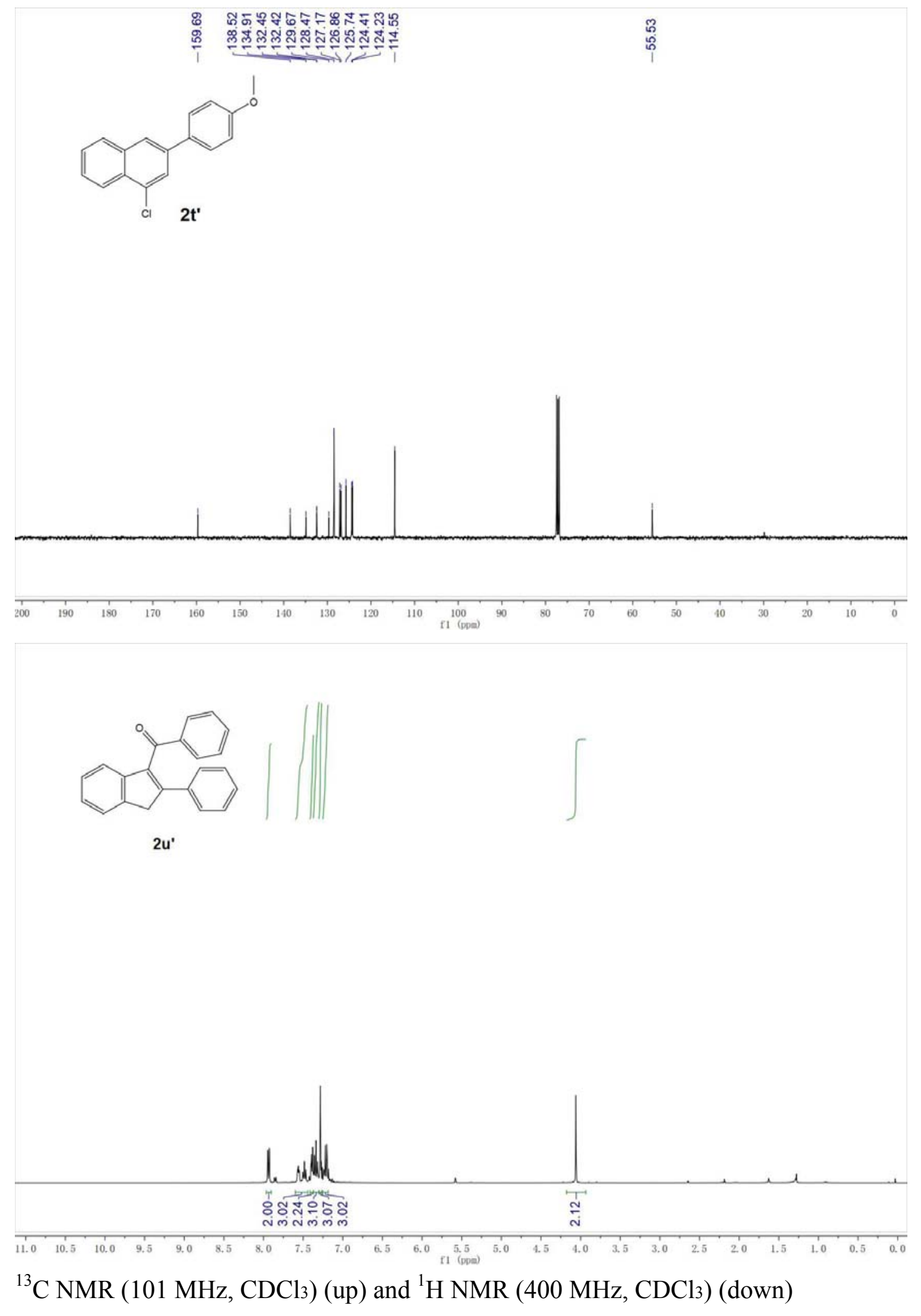



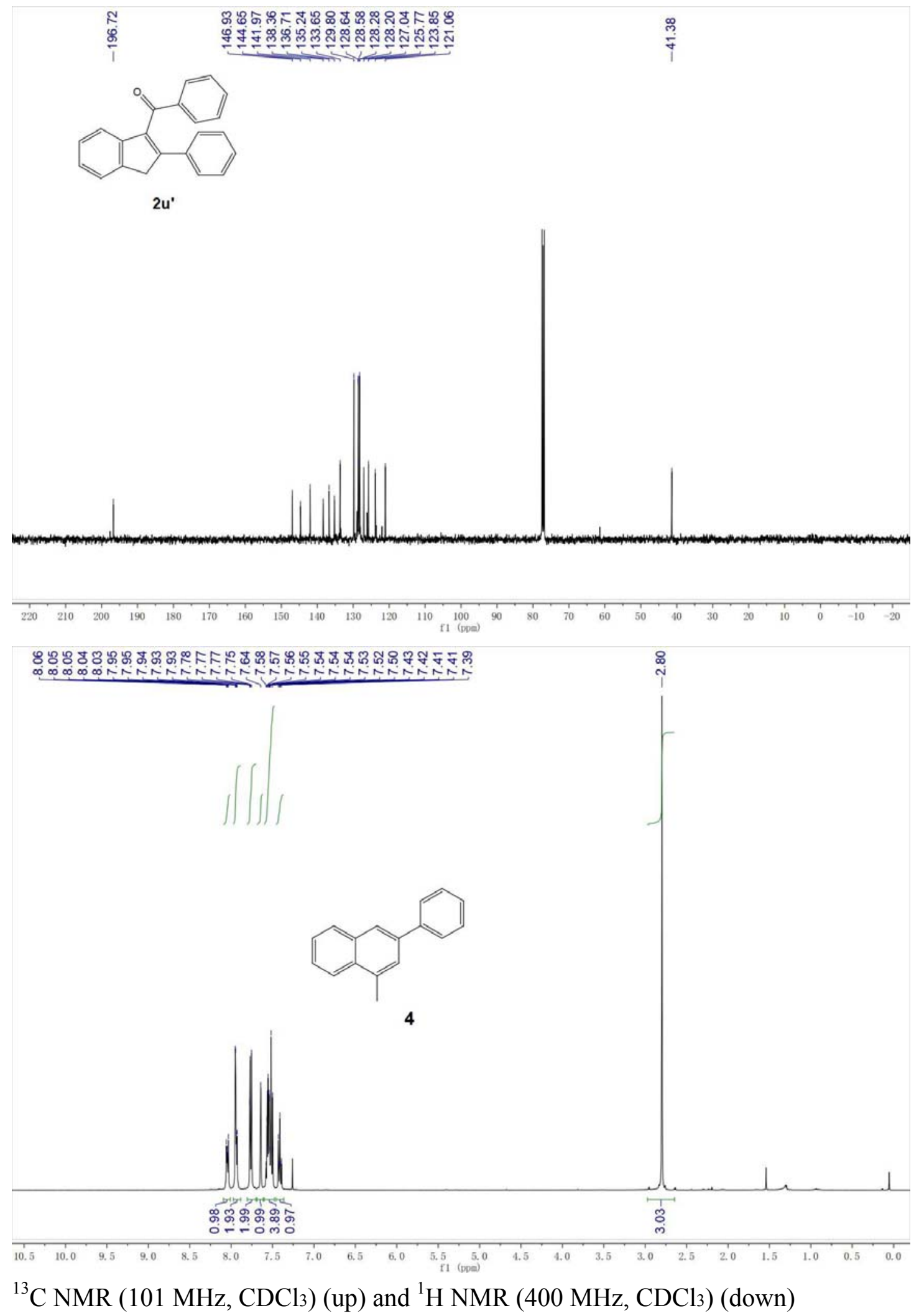

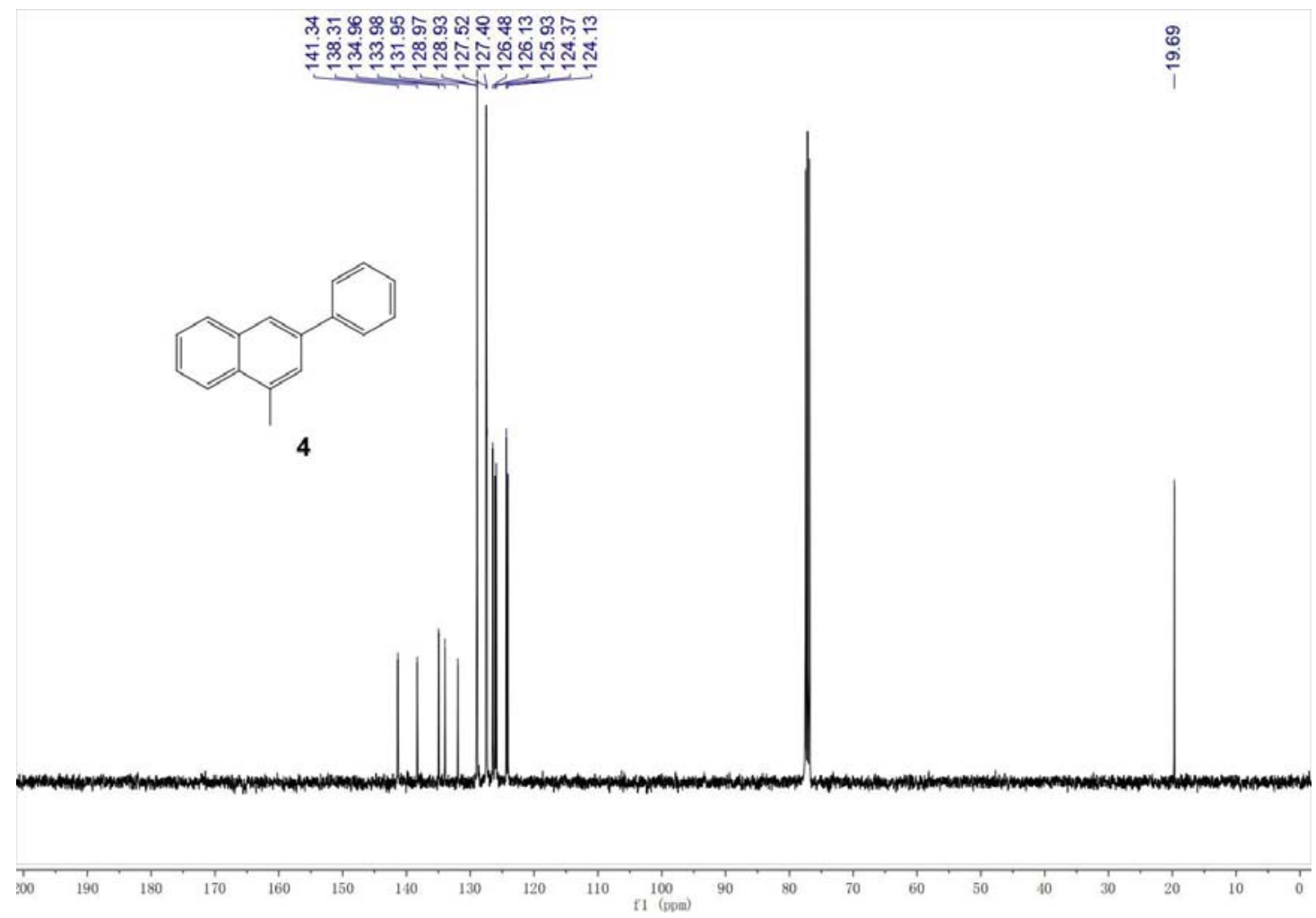

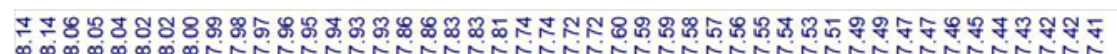

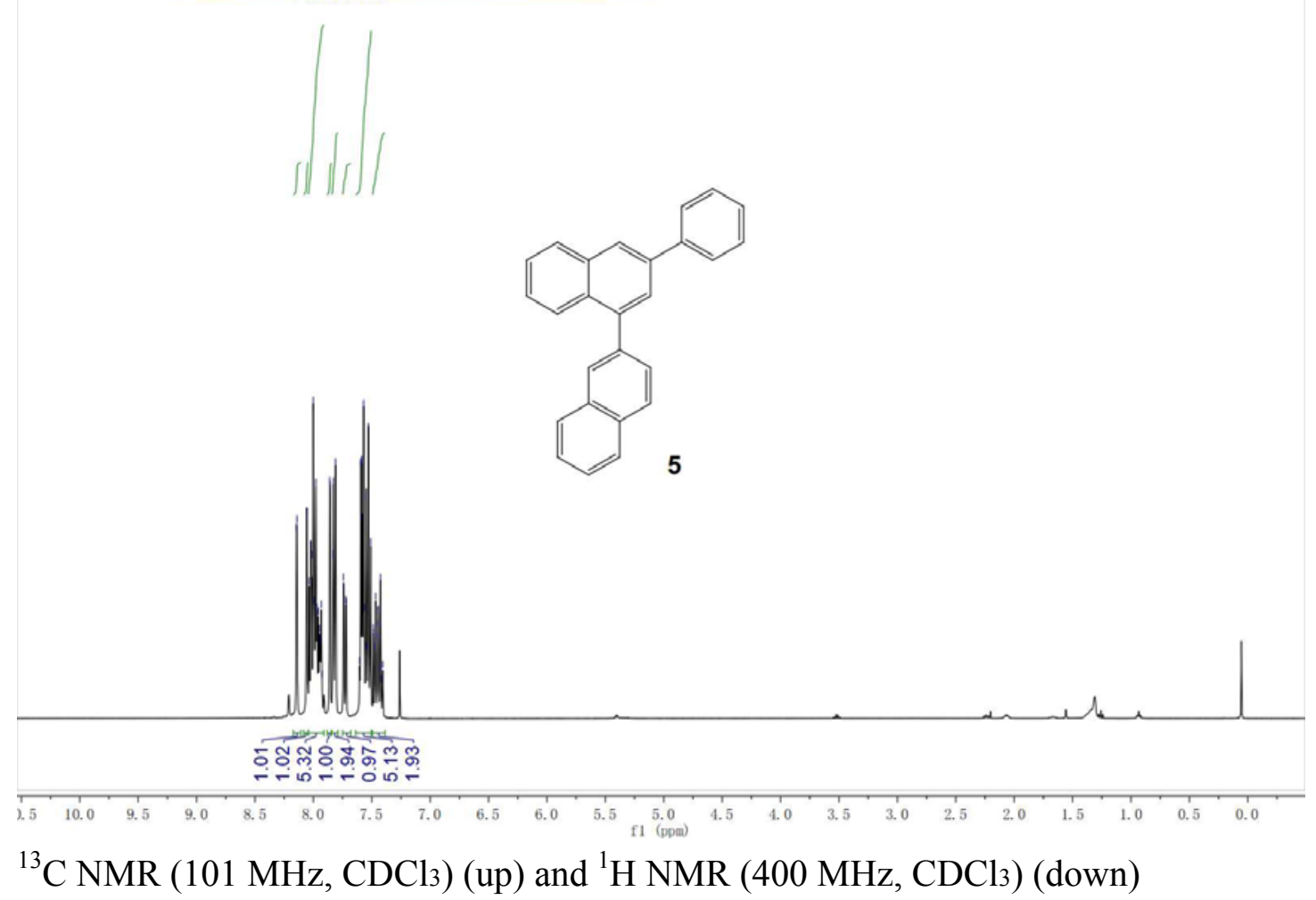



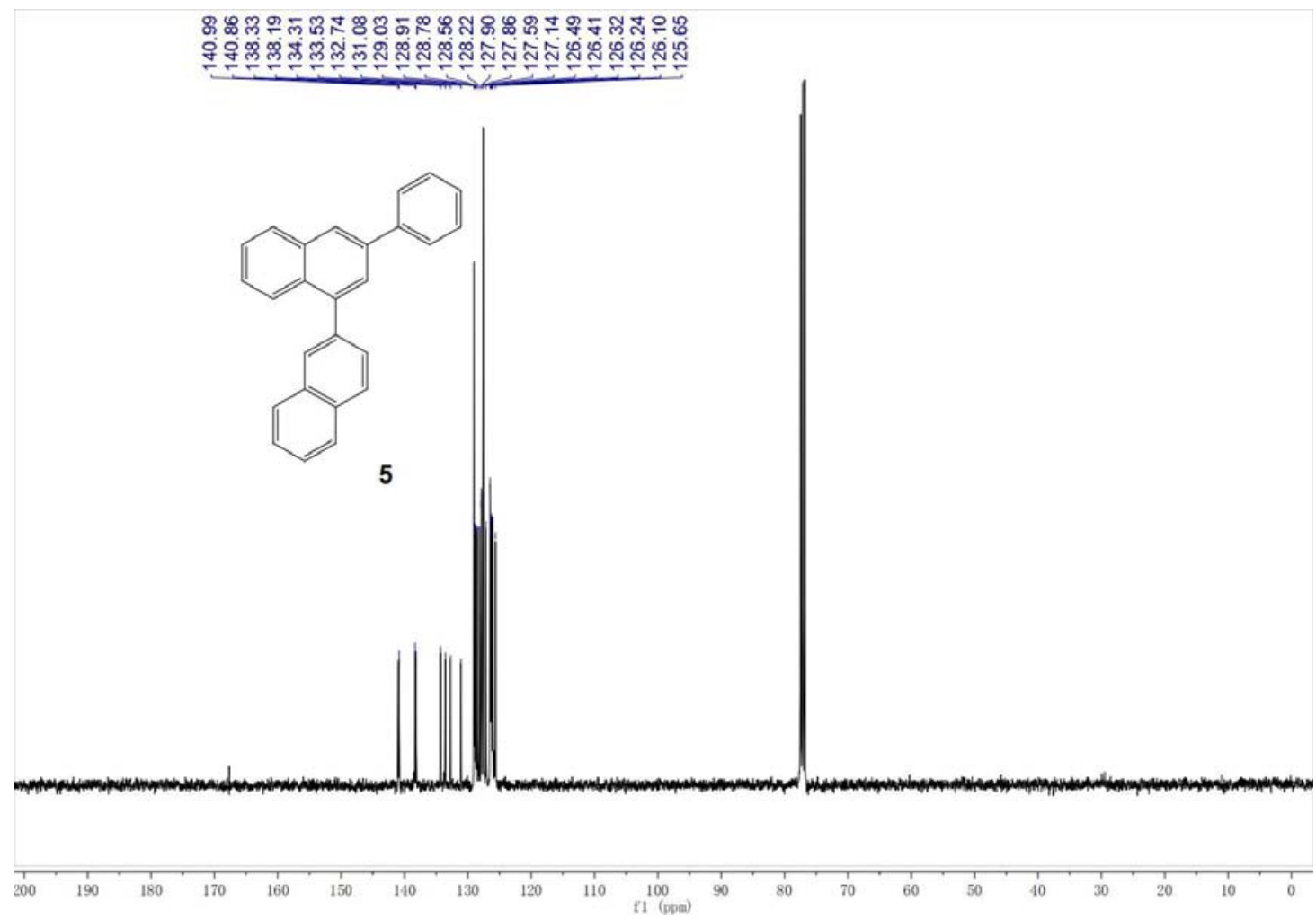

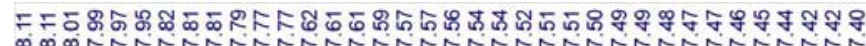

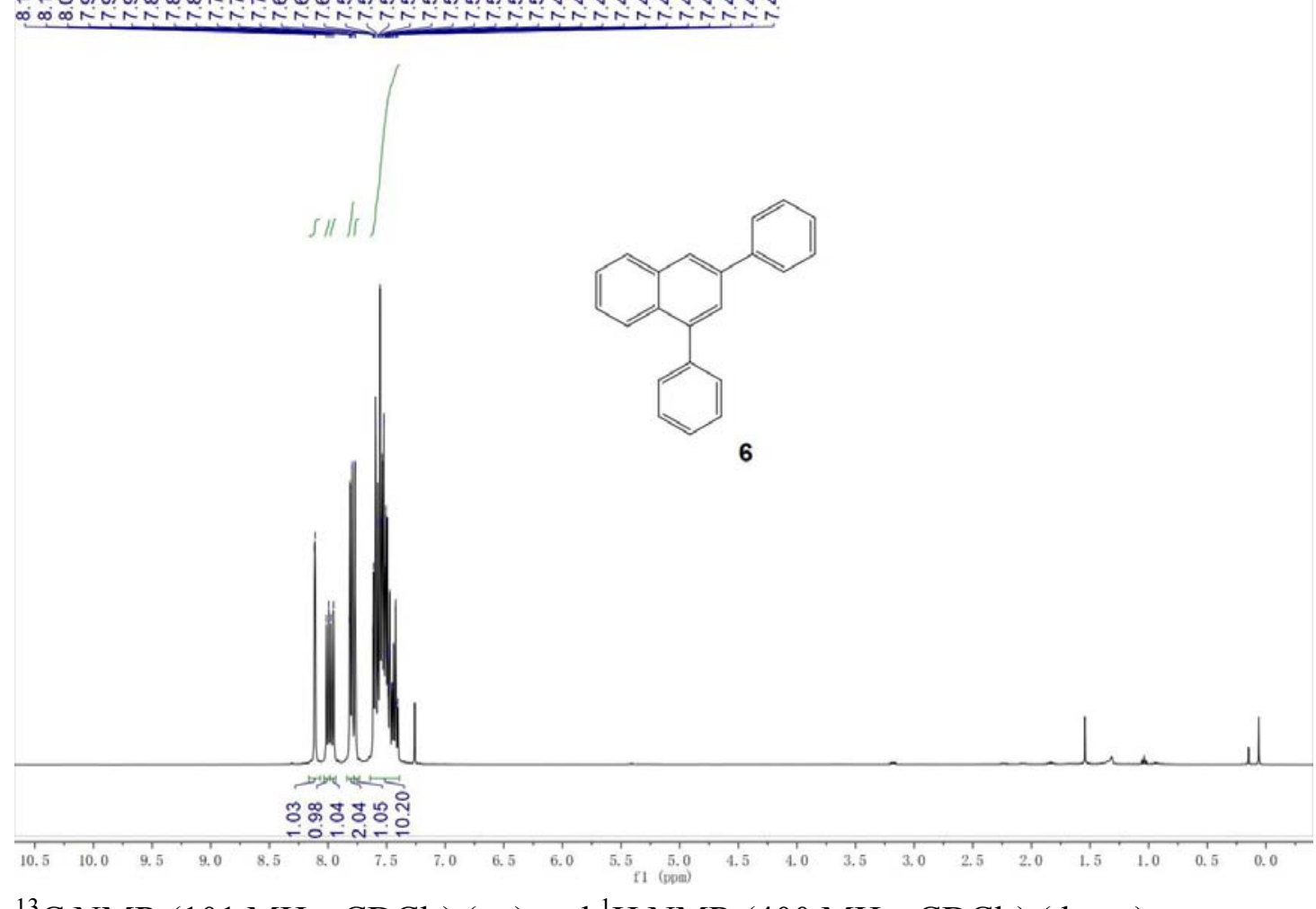

${ }^{13} \mathrm{C}$ NMR (101 MHz, $\mathrm{CDCl}_{3}$ ) (up) and ${ }^{1} \mathrm{H}$ NMR (400 MHz, $\left.\mathrm{CDCl}_{3}\right)$ (down) 

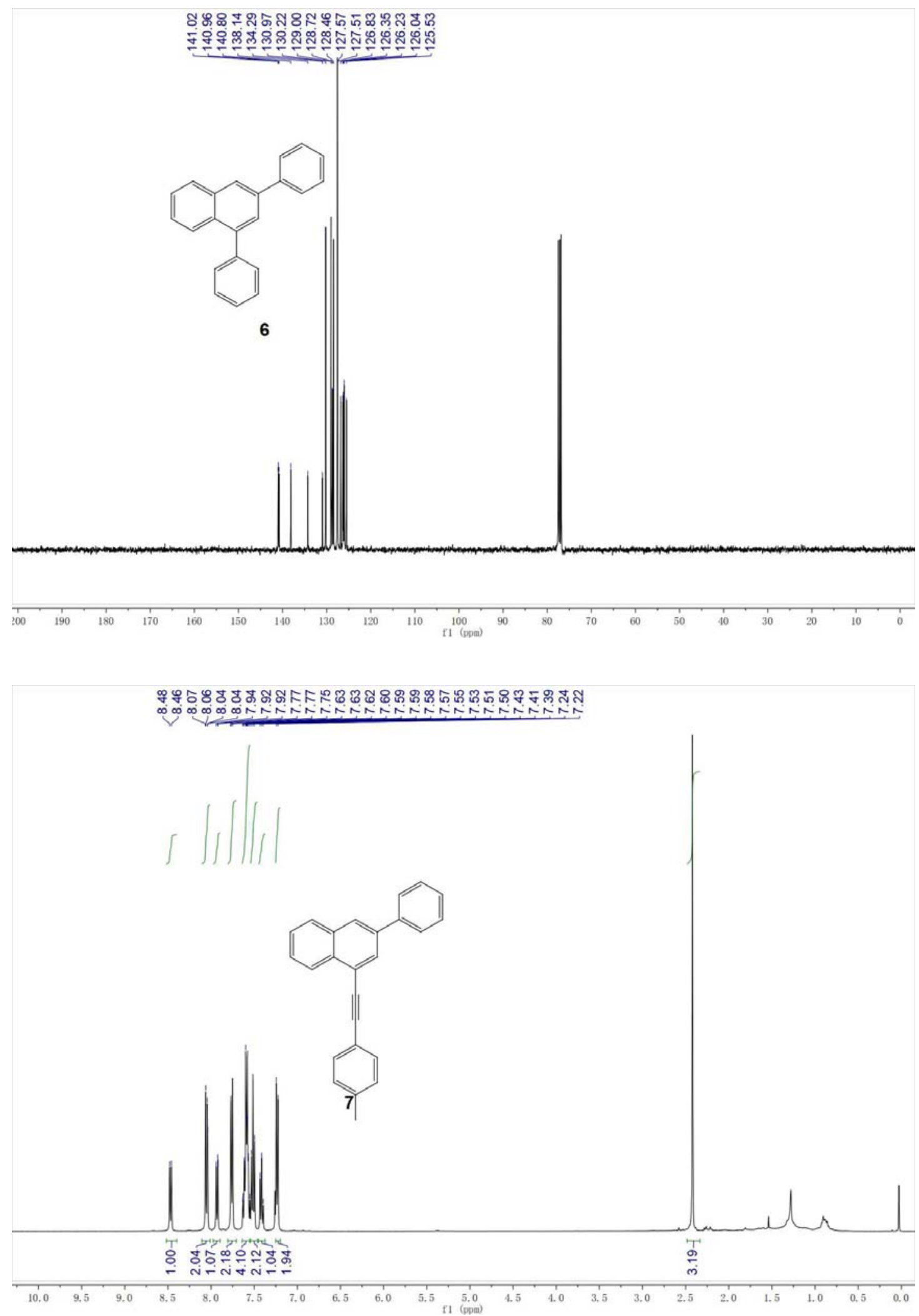

${ }^{13} \mathrm{C}$ NMR (101 MHz, CDCl 3 ) (up) and ${ }^{1} \mathrm{H}$ NMR (400 MHz, $\left.\mathrm{CDCl}_{3}\right)$ (down) 


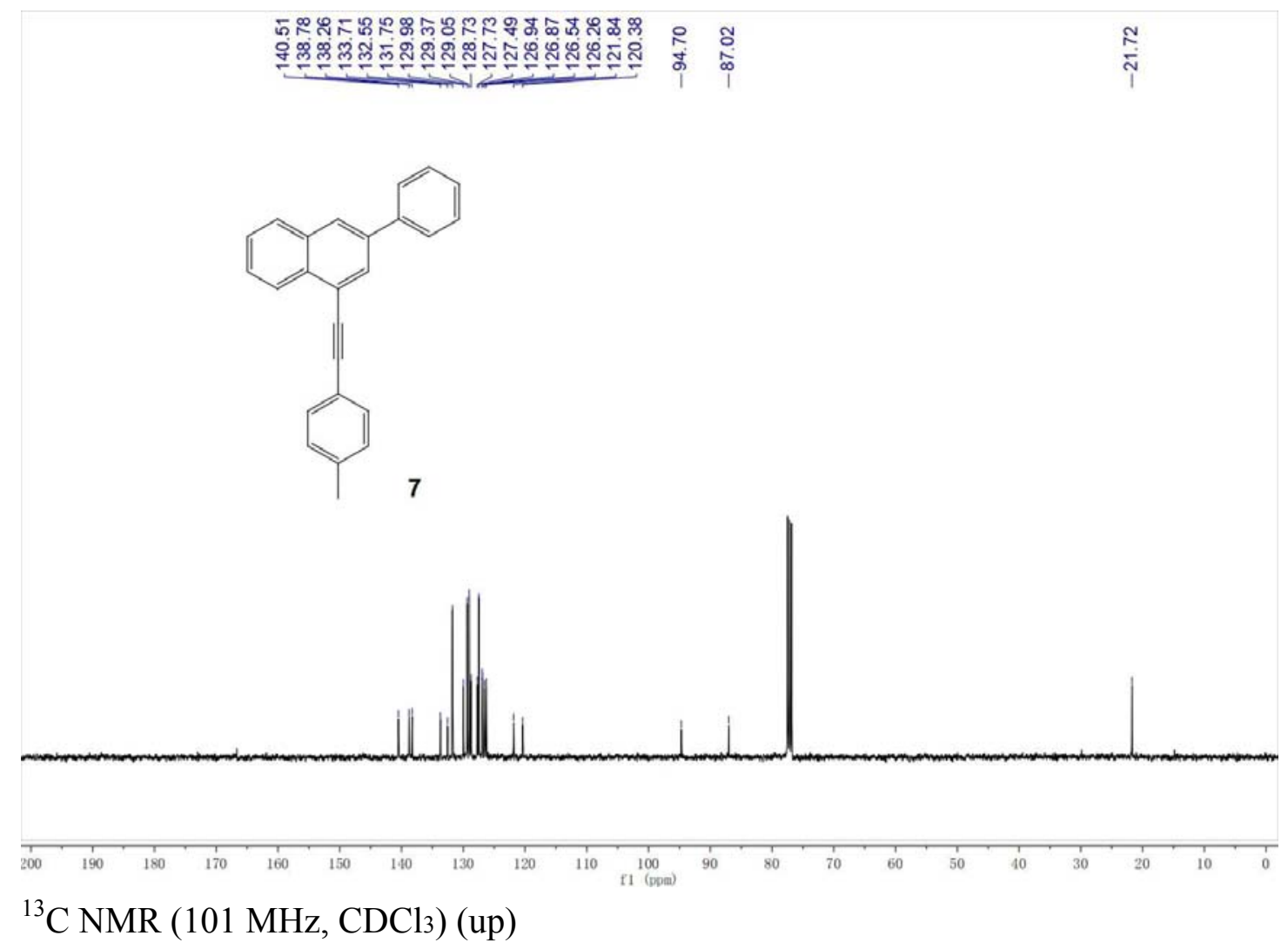

